\title{
Mechanical Properties of the Ich5 Organ in the Drosophila Larva
}

Dissertation for the award of the degree "Doctor of Philosophy" (Ph.D.)

Division of Mathematics and Natural Sciences of the

Georg-August-Universität Göttingen

submitted by

Achintya Prahlad

from Bengaluru, India

Göttingen 2017 
Members of the thesis committee:

Prof. Dr. Christoph F. Schmidt (Supervisor, $1^{\text {st }}$ reviewer)

Third Institute of Physics - Biophysics (DPI), Faculty of Physics, University of Göttingen

Prof. Dr. Martin C. Göpfert (2 ${ }^{\text {nd }}$ reviewer)

Schwann-Schleiden Research Center, Faculty of Biology, University of Göttingen

Dr. Claus Heussinger

Institute of Theoretical Physics, Faculty of Physics, University of Göttingen

Additional members of the examination committee:

Prof. Dr. Sarah Köster

Institute of X-ray Physics, Faculty of Physics, University of Göttingen

Prof. Dr. Jörg Enderlein

Third Institute of Physics - Biophysics (DPI), Faculty of Physics, University of Göttingen

Prof. Dr. Stefan Klumpp

Institute for Nonlinear Dynamics, Faculty of Physics, University of Göttingen

Date of the oral examination: 20 July 2017 


\section{Affidavit}

I hereby declare that this thesis has been written independently, with no other sources and aids than those quoted. It is based on my own work and has not been submitted for any other degree.

Achintya Prahlad

Göttingen, 14.05.2017 


\section{Contents}

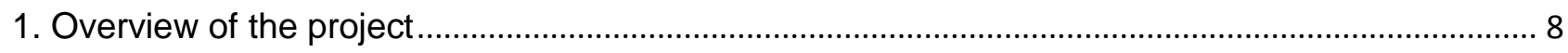

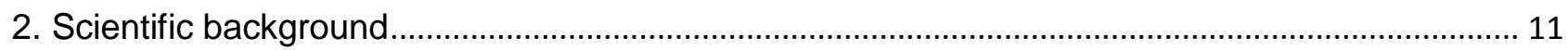

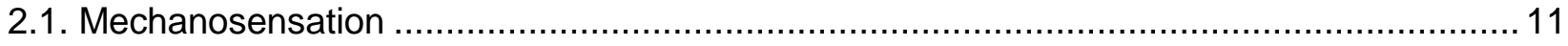

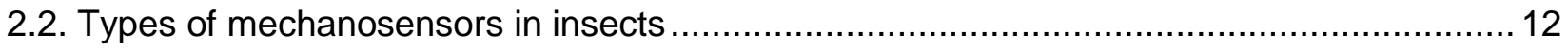

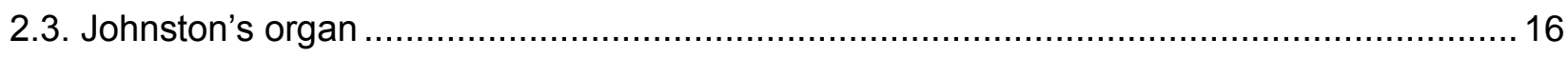

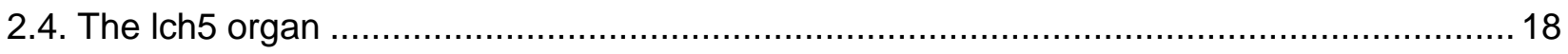

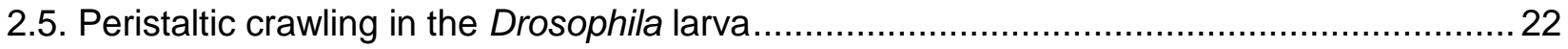

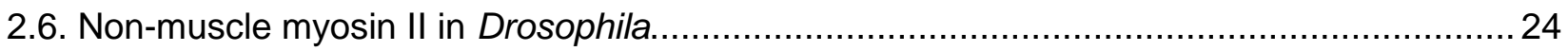

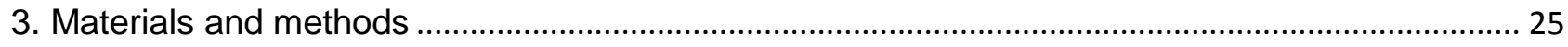

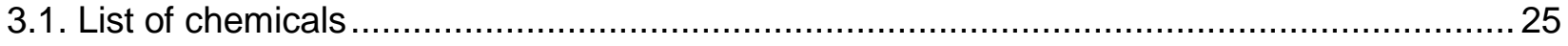

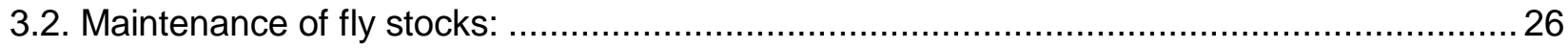

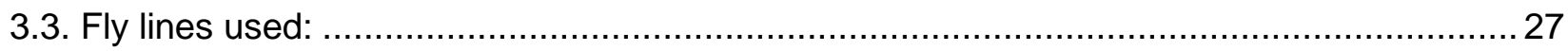

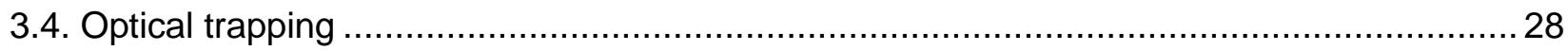

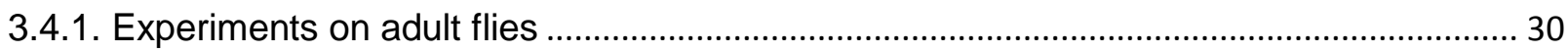

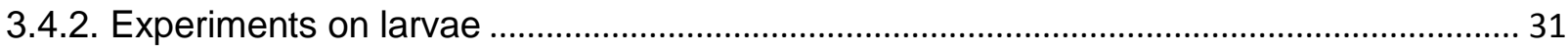

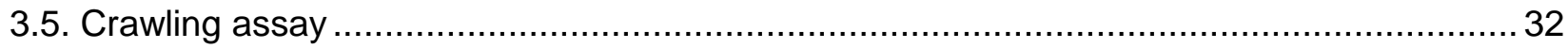

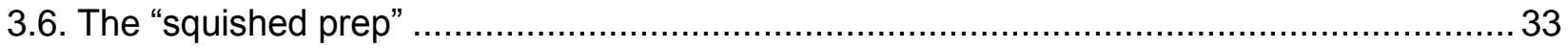

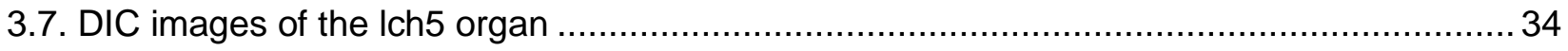

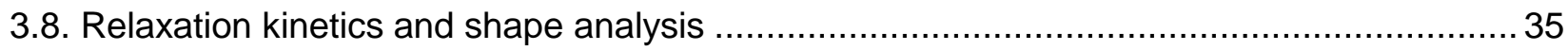

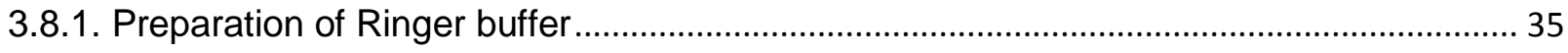

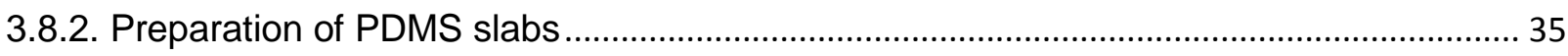

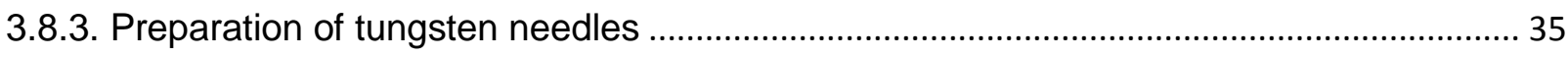

3.8.4. Preparation and dissection of Drosophila larvae ........................................................... 37

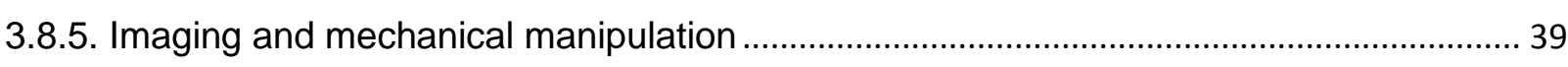

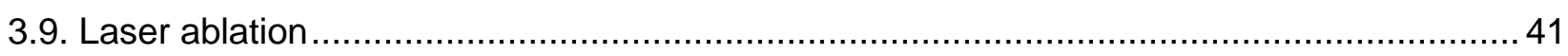

3.10. Force measurements using calibrated needles .................................................... 45

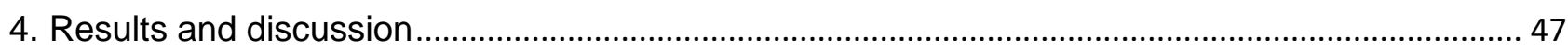

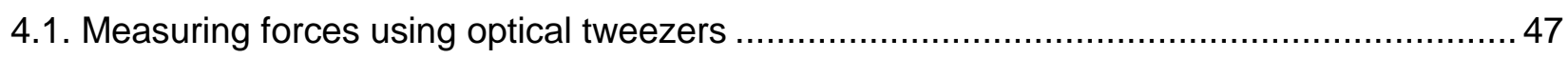

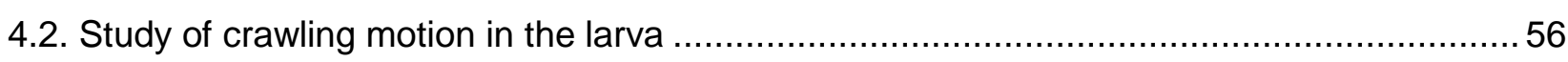

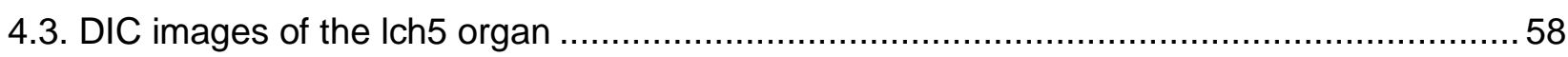

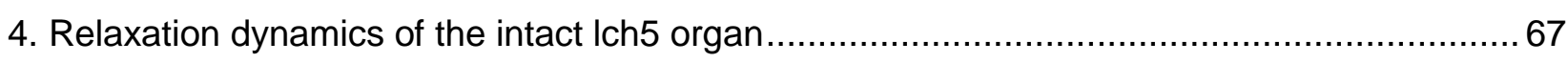

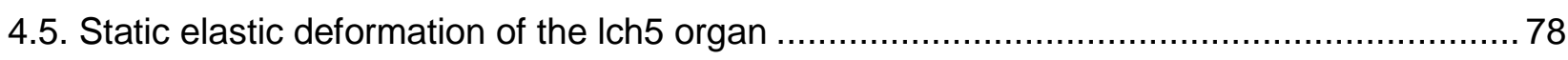

4.6. Relaxation of the Ich5 organ after laser ablation .................................................... 82

4.7. Measurement of forces using calibrated glass pipettes............................................. 89 


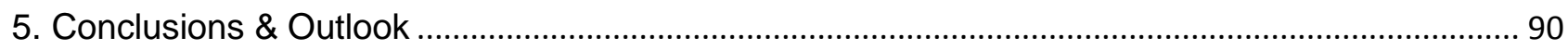

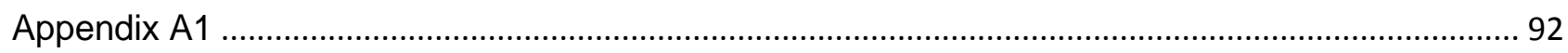

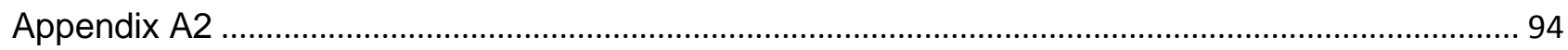

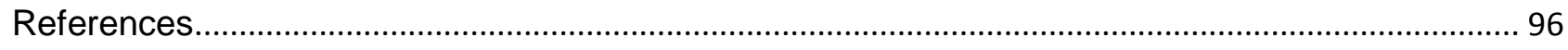

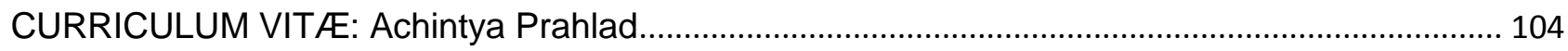




\section{Acknowledgements}

First and foremost, I would like to express my gratitude to my PhD supervisor, Christoph Schmidt, for giving me a chance to work on this fascinating topic, and for his constant guidance and help right from the beginning. This project would have certainly not been possible without him. I am also grateful to Martin Göpfert and Claus Heussinger, the other two members of my thesis advisory committee, for their advice and support. Prof. Göpfert has been our main collaborator on this project, and for this reason I am highly grateful to him for his vital contribution to my project and for allowing me the use of his lab facilities. I am also grateful to Jörg Großhans and Oliver Bäumchen for permitting me to work on some of my experiments in their respective labs (laser ablation at the Großhans lab, and the glass needle experiments at the Bäumchen lab). I am also grateful to Florian Rehfeldt, Dieter Klopfenstein and Jörg Enderlein for their support.

I am also grateful to Christopher Battle and Alok Daniel Wessel for training me in optical trapping techniques at the beginning of my PhD and for their help during experiments, Christian Spalthoff and Ben Warren for training me in several important techniques in Drosophila biology, Bart Geurten for providing his custom-designed machine for sharpening tungsten needles, Deqing Kong for his active participation in the laser ablation experiments, Christian Kreis for working together with us on the glass needle experiments, Chonglin Guan for his valuable inputs in the glass pipette and laser ablation experiments. I thank all my colleagues at the department for being there for me these four years and making me feel at home in the lab: Nikta Fakhri, Meenakshi Prabhune, Galina Kudryasheva, Samaneh Rezvani, Constantin Kohl, Kengo Nishi, Carina Wollnik, Jianguo Zhao, Christina Jayachandran, Miquel Banchs i Piqué, Ulrich Fromme, André Düselder, Florian Schlosser, Alice Wiesbaum, Paula Sanchez Baeza, Mitja Platen, Knut Heidemann, Nuria Gavara, Peter Weist, Renata Garces, Philipp Linke, Philipp Hannibal, Daniel Meyer, Daniel Haertter, Amna Khalid, Theresa Kaufeld, Theresa Hoppe, Behzad Golshaei. Many thanks are due to everyone else at DPI - Nicole Rehbein, Elke Zech, Ursula Hahn-Wörgötter, Sabine Huhnold, Thomas Geiling Markus Schönekess, Dieter Hille, Kerstin von Roden, Tanja Gall, Ulrike Schulz, and many others.

I am thankful to my family and to all my friends in Göttingen and elsewhere for standing by me and giving me the strength to finish this $\mathrm{PhD}$. I am also grateful to anyone else whose name I may have missed in this list.

Achintya Prahlad

Göttingen, 14.05.2017 


\section{Overview of the project}

I begin with a brief account of the topic of mechanosensation, followed by an outline of how the project evolved. The experimental techniques used and their rationale are described. The section ends with a short description of the thesis structure.

Out of all sensory modalities, mechanosensation is of special interest to biophysicists, since its transduction mechanism in most cases involves a direct mechanical gating of ion channels, as opposed to gating by a chemical messenger. One important class of mechanosensory organs are the chordotonal organs. These perform proprioceptive and other mechanosensory functions in insects and crustaceans. There is a wide diversity of chordotonal organs across species and also within a single species, but there is a great deal of structural overlap. This makes them rather interesting to study, because results obtained by studying the mechanics of one type of chordotonal organ can in principle be applied to others. The mechanical properties of these organs are thought to be correlated to the sensory functions. Mutant studies, laser Doppler vibrometry and other techniques have given us some information on the functioning of these organs, but direct mechanical probing of their components had hitherto not been carried out. This was the motivation for my project, in which I measured mechanical properties of a particular chordotonal organ - the lateral pentascolopidial (Ich5) organ - that plays a key role in proprioceptive locomotion control in Drosophila larvae.

In the early stages of the project, the mechanical properties of Johnston's organ - the antennal hearing organ in the adult fruit fly Drosophila melanogaster - appeared attractive, because it functions using an active process very similar to that operating in the vertebrate ear. The idea was to measure active fluctuations from the sensory cilia of the organs, which are believed to be the main transducing element. This would involve initially measuring forces at the arista, which is the external sound receiver of the antenna, and then moving inwards. Attempts to measure forces at the arista using optical tweezers were unsuccessful owing to thermal damage. There were also other difficulties, mainly that optical trapping requires a water sample and the fly does not survive under water. We also understood that probing the internal structures of Johnston's organ would not yield conclusive results, since this would require perforating the cuticle and would impact the mechanics. We then shifted our attention to the Ich5 organ since it is also a chordotonal organ, albeit simpler in structure than Johnston's organ. We first decided to repeat the bead-trapping experiment on the Ich5 organ. For these experiments I used a dissected fillet preparation of the larva. Here the muscles presented an obstacle to bringing the bead in contact with the organ. This was overcome by digesting the muscles with collagenase. However, once the bead was stuck to 
the organ it could not be trapped, and no fluctuations could be measured. Since the ciliary mechanics appeared inaccessible, we shifted to whole-organ mechanics.

Since the Ich5 organ is believed to regulate the crawling mechanism, we decided to focus on this aspect. We captured detailed videos of the deformation of the cuticle as the larva crawls. Also, I obtained high-resolution images of the Ich5 organ using DIC microscopy and a self-designed preparation. This involved flattening the larva between a slide and a coverslip, such that the gut was squeezed out and the sample was rendered transparent. This "squished prep" proved highly useful to us in the laser ablation experiments that we performed in the later stages of the project.

The next set of experiments was to measure the mechanics of the lch5 organ in a fillet prep using a tungsten needle. We applied tension to the whole organ in situ by transverse deflection. Upon release of force, the organ displayed overdamped relaxation with two widely separated time constants, tens of milliseconds and seconds respectively. When the muscles covering the lch5 organ were excised, the slow relaxation was absent and the fast relaxation became faster. We also observed the change in shape of the organ as it was deformed by the needle. A cusp-like shape was seen. The ends of the organ were fixed during the entire process, which meant that the length of the organ increased, and once the needle was released, relaxed back to its original value. Interestingly, most of the strain in the stretched organ is localized in the cap cells, which account for two-thirds of the length of the entire organ, and could be stretched to nearly a $10 \%$ increase in length without apparent damage.

Next, laser ablation experiments on the lch5 organ were carried out. For this, the earlier mentioned "squished prep" was employed. Using a UV laser, the organ was then severed at different points, and its retraction was observed. It was found that cap cells retracted by over $100 \mu \mathrm{m}$ after being severed from the neurons, indicating considerable steady state stress and strain in these cells. Also, in a myosin knockdown mutant, a much smaller retraction in comparison to the control was seen. Given the fact that actin as well as myosin motors are abundant in cap cells, the results point to a mechanical regulatory role of the cap cells in the Ich5 organ, and a significant contribution of myosin motors to this process.

The final set of experiments was to develop a technique to measure forces in the lch5 organ using calibrated glass needles. We have optimized the method and made it suitable for future investigations. 
This thesis is structured as follows:

Scientific Background gives a short introduction to mechanosensation, followed by a description of mechanosensory organs in insects with special reference to chordotonal organs. Next, Johnston's organ, the Ich5 organ, and the crawling mechanism of the Drosophila larva are described, along with a short note on non-muscle myosin II, a molecular motor that is important in many organs of Drosophila including in the lch5 organ.

Materials and Methods describes in detail the various techniques and materials that have been used for this research.

Results and discussion details the results obtained from our experiments and the inferences drawn from them. The results from the relaxation kinetics and shape experiments, as well as the laser ablation experiments, were submitted for publication to the Biophysical Journal in March 2017, as follows: Prahlad, A., C. Spalthoff, D. Kong, J. Großhans, M. C. Göpfert, and C. F. Schmidt. 2017. Mechanical properties of a Drosophila larval chordotonal organ. The manuscript is currently under revision. Some text in this thesis has been quoted verbatim from the paper (details in Appendix A2).

Conclusions and outlook discusses next steps in this particular project, and suggests possible future directions in this line of research. 


\section{Scientific background}

\subsection{Mechanosensation}

Mechanosensation (1) is one of the most basic sensory modalities, found in all living organisms ranging from minute bacteria to colossal blue whales. It involves processing of mechanical stimuli, as opposed to optical, electrical or chemical ones. It is important to study because it has farreaching applications. Examples are understanding how cancer cells migrate in order to prevent metastasis (2), understanding locomotion in insects for the purpose of designing robots modeled on them (3), dissecting the mechanism of hearing to produce improved hearing aids (4), et cetera. If we compare mechanosensation to, say, vision or olfaction, we see that not only are the stimuli different, but the transduction mechanism - i.e. the mechanism by which a sensory stimulus is converted to an electrical signal (5-12) or in some cases leads to gene regulation $(13,14)$ - also differs. In vision, a light stimulus changes the conformation of rhodopsin, which eventually results in secondary messengers being activated and ion channels opening (7-10). In chemosensation, which includes olfaction (11) and gustation (15), the ion channels are either directly activated by an odorant or taste agent, or it could also be that the stimulus molecules bind to receptors that then activate the channels. However, in mechanosensation, it is the mechanical stimulus itself that gates the ion channel $(1,6,16-18)$. A good example is the mscL channel in the bacterium Escherichia coli $(6,17)$. When the bacterial cell is placed in a hypo-osmotic environment, water flows into it and it begins to swell. This deformation of the membrane opens the mscL channels, and excess solutes are removed via the channel, in order to prevent cell wall rupture and bring the organism back to its original state. Another important difference between some mechanical senses (especially the auditory sense) and other modalities such as vision (7-10) and olfaction (11) is the following: the amplification of the signal takes place actively and mechanically $(5,12,19-25)$, as opposed to a chemical amplification that would take place e.g. by a large number of secondary messenger molecules being activated by one rhodopsin molecule in vision (26). An active process is defined as one that takes place through the sustained conversion of stored energy, usually chemical or electrical, into movement. For example, stereocilia in hair cells in the vertebrate ear spontaneously generate additional forces through the action of the motor proteins prestin

(19) and myosin. Passive processes include fluctuations due to thermal energy or the response to externally imposed forces (27).

What constitutes a mechanical stimulus? The answer to this question is different for different organisms. For a single cell, these typically include turgor pressure (6), stiffness of substrates (28), mechanical obstacles (29), or liquid flow (27). For a plant, it could be wind direction (30), touch (31), gravity (32), or bending of the stem (33). In animals, the following senses are most commonly talked 
about: touch, hearing (5, 12, 19-25), mechanical pain, balance, and proprioception (34-36). Proprioception can be explained as gauging the relative positions of body parts. It shall subsequently be discussed in some detail in section 2.5, since it forms the basis for the mechanism of locomotion of our animal of interest - the Drosophila larva $(35,37-44)$. But before that, let us have a look into the diversity of mechanosensory organs and their functions in this and other insects.

\subsection{Types of mechanosensors in insects}

Insect mechanosensory organs can be classified as ciliated and non-ciliated. The main types of nonciliated organs are the multipolar receptors $(45,46)$ and campaniform sensilla (47-49). Ciliated mechanosensors can be further divided into tactile hairs $(46,50,51)$ and chordotonal organs $(46$, $50,52)$. Out of all these categories, I will be concerned mainly with the ciliated organs, and specifically the chordotonal organs, which we shall discuss in greater detail.

Multipolar receptors consist of single multidendritic neurons that innervate diverse tissues and internal organs $(45,46,53-56)$. They perform a variety of functions, e.g. regulation of gut contractions in the blowfly $(57)$, or regulation of terrestrial and aerial locomotion in moths $(54,55)$ or locusts $(53$, 56). Some multipolar receptors in the Drosophila larva are involved in nociception $(46,58)$, and others in proprioception $(46,59)$.

Campaniform sensilla (47-49) are rounded internal organs that respond to forces within the body wall of insects. Their basic structure is a dome-like apparatus that is innervated by one bipolar neuron (60-62). They are involved mainly in the regulation of walking $(61,63)$ or flight $(60,64)$. Measurements of decrease in the strain compliance upon application of highly directed mechanical stimuli using a piezo-controlled tungsten needle in the campaniform sensilla of the cockroach Blaberus discoidalis have been described (65).

Tactile hairs are found all over an insect's body (66). The most commonly studied tactile hairs are the mechanosensory bristles of Drosophila (67). The construction is of a hollow shaft - the hair protruding outward from the cuticle, with a monodendritic neuron at its base. The neuron terminates in a cilium, which is the main element in the mechanotransduction process. When the shaft is deflected, it displaces the tip of the neuron, causing mechanosensory channels to open and an ion current to flow into the neuron (46).

Chordotonal organs are a family of specialized mechanoreceptors that are found in all insects, and also in crustaceans, where they perform a variety of sensory functions $(38,42,44,49,50,52,68$ - 
70). They are unique to these two classes of arthropods (50). They generally are stretch receptor organs, bound into proprioceptive or other specialized mechanosensory organs $(50,52)$. Examples of proprioceptive chordotonal organs are the lateral pentascolopidial chordotonal organ (Ich5 organ) in the body wall of the larva of the fruit fly Drosophila melanogaster (44, 49, 71-73), the femoral chordotonal organ in the leg of Locusta migratoria (50) etc. In Drosophila, Ich5 organs are suspended between two attachment points at the cuticle, one on each side of the dorsal half of the larva in each segment in a tense straight conformation. The lch5 organs do not appear to be directly coupled to contractions of individual muscles. Rather, they sense deformations of the cuticle caused by muscle contraction - which is then transduced into a neural signal via the sensory neurons that are part of the organ. This finds mention in Halachmi et al., 2016 and Klein et al., 2010, though references for this have not been cited by them $(71,72)$.

Literature from the late 19th and early 20th century (74-76) shows that the name "chordotonal organ" arose from a belief that these organs oscillate like the strings of a musical instrument. However, it is likely that this is not the case, given the degree to which the organ is damped by the embedding muscle tissue and the haemolymph.

The basic unit of a chordotonal organ is the scolopidium (Fig. 2.4.1) (25, 44, 49, 50, 52, 77). The number of scolopidia in chordotonal organs varies, as does the number of neurons in a scolopidium, depending on the organ and the insect species. The antennal auditory organ (Johnston's organ) of the adult fruit fly Drosophila melanogaster, contains around 250 scolopidia $(49,77)$, with two or three neurons in each - while the Ich5 or pentamere organ of the larva of the same species, consists of five scolopidia $(44,49)$, each containing a single neuron. A scolopidium consists of 1 to 3 sensory neurons, integrated into the following accessory structures: one ligament cell forming one connection to the cuticle via an attachment cell, one cap cell forming the other connection to the cuticle via another attachment cell, and one scolopale cell from which a capsule called the scolopale emerges. The neurons each possess a single dendrite that terminates in a sensory cilium consisting of 9 peripheral microtubule doublets without a central doublet $(25,52)$. The cilium is activated by dyneins (78). These are divided into two categories: modulators and force generators. They have been shown to be involved in promotion and regulation of active amplification. The cilia in each scolopidium are enclosed in the scolopale (Fig. 2.4.1), which is comprised mainly of a dense actin cytoskeleton including a few microtubules. In scolopidia containing more than one neuron, all sensory cilia are enclosed in the same scolopale. The fluid in the scolopale cavity has a high concentration of potassium ions, similar to the endolymph in the inner ear of vertebrates $(25,52,77)$. Attached to the apex of each scolopale is a cap cell, a strongly elongated cell that, in the case of the Ich5 organ, accounts for more than two thirds of the total length of the organ. This cell is connected to the scolopale by a structure known as the dendritic cap, which is conspicuously visible in electron microscopy (52). On the opposite end of the organ, closer to the initial segment of the neuronal axon 
is the ligament cell, which is much shorter than the cap cell in the lch5 organ. The cap cells and ligament cells are both firmly fixed to the cuticle by means of attachment cells $(69,71,72)$ (Fig. 2.1). The Ich5 organ has one attachment cell for all five ligaments, and one attachment cell for all five cap cells (69).

In some chordotonal organs such as Johnston's organ (JO), the hearing organ in the adult fruit fly Drosophila, the sensory cilium contains an accumulation of intraflagellar transport proteins known as the ciliary dilation, which is believed to have a strategic role in the transduction process $(79,80)$. The dilation helps maintain the compartmentalization of two distinct classes of mechanosensory channels, whereby the key channel NOMPC is confined to the apex of the cilium (80).

Almost 90 years ago, in 1931, Heinrich Hertweck published a thesis (49) that describes the detailed structures of several different classes of chordotonal organs in Drosophila, from the embryo to the adult. In more recent times, there have been numerous discoveries about the sensory function of two chordotonal organs in Drosophila: Johnston's organ (JO), the hearing organ in the adult fruit fly, and the Ich5 organ, the larval proprioceptor studied in this thesis $(22,24,25,44,46,73,77,78,81$, 82).

What is lacking is detailed information about the internal mechanics of chordotonal organs, i.e. which parts of the scolopidia deform and how when impacted by a mechanical stimulus, and how these deformations activate ion channels and are transduced to a neural signal. Field and Matheson mention in their 1998 review (50) that the femoral chordotonal organ in the locust Locusta migratoria obeys Hooke's law within an elastic limit, but do not provide quantitative information. They suggest that the elongated cap cells, which they refer to as the attachment cells, must play a pivotal role in the transduction mechanism, and suggest that the mechanism must involve microtubules sliding past one another. Measurements of the response of the femoral chordotonal organ in the stick insect to electrical stimulation (83), and the impact of changes in length on the tension of the muscle adjoining a stretch receptor organ in the crayfish Pacifastacus leniusculus (84), have also been reported.

Direct measurements of the compliance of the chordotonal organs of Drosophila have, to our knowledge, not yet been reported. Drosophila is a particularly useful animal model (85), due to its ease of availability and maintenance. Its short life cycle affords greater speed in generating mutant and transgenic lines as compared to vertebrate models. Also, many of its physiological processes have analogies with vertebrates. An advantage specific to our project is that the Drosophila larva is transparent and the chordotonal organs are readily visible in a "squished prep" (section 3.6), and can also be directly probed in a fillet preparation (43). 


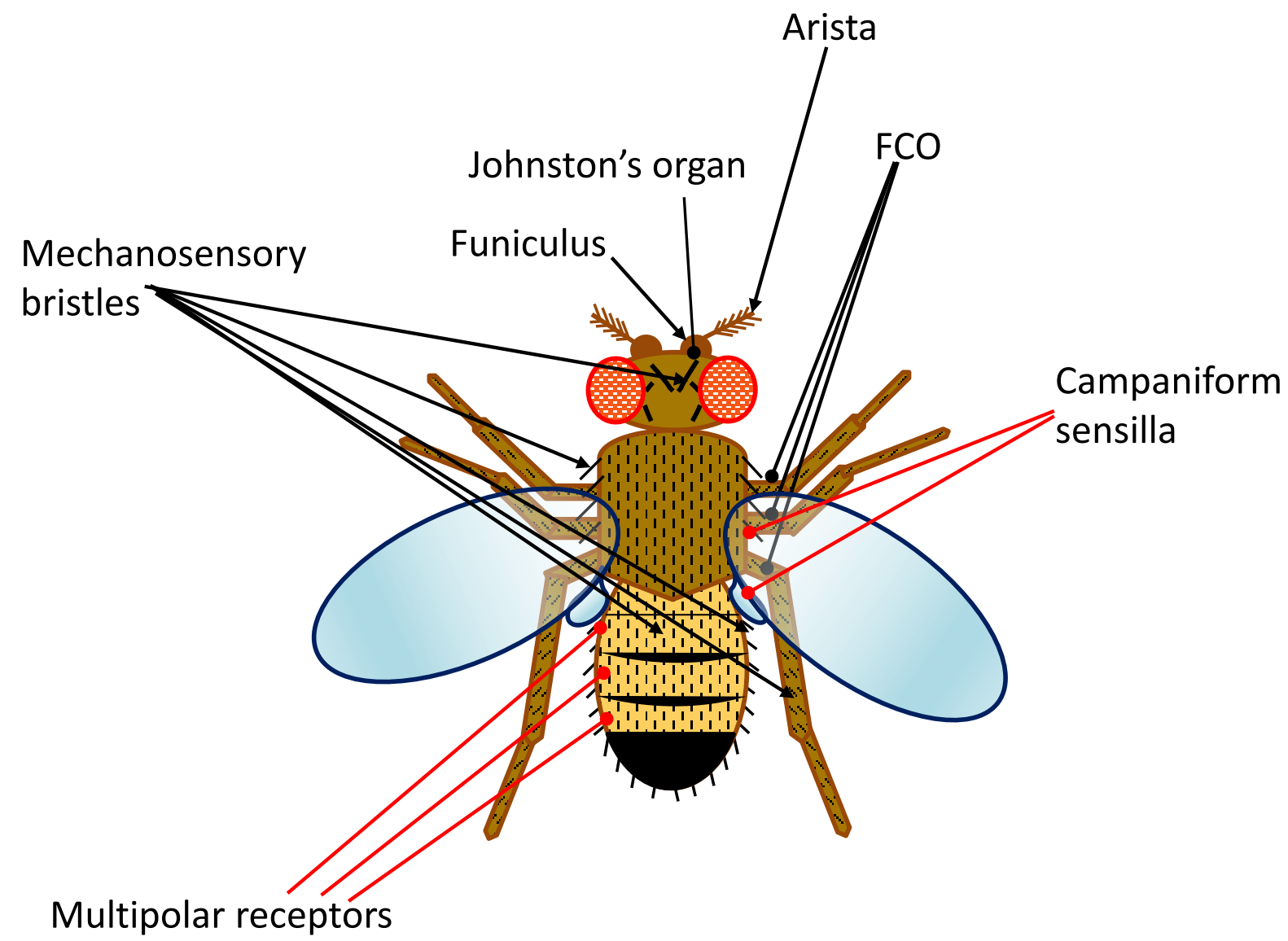

Fig. 2.2.1. Diagram showing the positions of some important mechanosensory organs in the adult fly Drosophila (46, 50, 77, 86-88). 


\subsection{Johnston's organ}

J. E. Yack, in her 2004 review on auditory chordotonal organs (52), states that the line between auditory and proprioceptive organs is often very thin - since many proprioceptors respond to sound stimuli (89), and proprioceptive signals could often occur within the response range of auditory organs $(90,91)$. This ambiguity, according to her, can be resolved by choosing to define an auditory organ as that which produces a coherent behavioral response to a sound stimulus.

Johnston's organ (JO) is an exteroceptive chordotonal organ present at the base of the antenna, in a region known as the pedicel $(12,25)$. Its auditory function has been studied extensively in Diptera such as drosophilid flies $(25,77)$ and mosquitoes $(92,93)$, and Hymenoptera such as honeybees (94). It can also act as a gravity sensor, e.g. in Drosophila (95, 96). In Drosophilae and mosquitoes, its predominant function is to detect what are known as "courtship songs". These are characteristic sound patterns emitted from the wings of a fly of one sex to attract an individual of the opposite sex for mating. In Drosophilae, the courtship song is produced by the male (97), while in mosquitoes it is the female that produces it (93).

Each Drosophila species has a song pattern that is unique to that species. It may often be the case that many species of fruit fly congregate in the same place, such as around a rotting fruit. It is of prime importance for a Drosophila female to identify the male of the same species, since crossspecies mating would produce sterile offspring. The antennae of the female are uniquely tuned to the song frequency of conspecific males (97), unlike in mosquitoes where it is the male that detects the song of the female (93).

The auditory apparatus in Drosophila, and also in other insects that possess JOs, consists of the entire antenna $(25,77,98)$. The external sound receiver is the outermost portion of the antenna - a feathery extension known as the arista, described as a "sail" by A. Manning in 1967 (98). This is attached to a pear-shaped segment called the funiculus. The funiculus is in turn pivoted in a depression in the pedicel. The chordotonal neurons of $\mathrm{JO}$ are arranged with their cilia in direct contact with this pivot. There are two opposing groups of neurons, arranged at an obtuse angle to each other. The total number of neurons is approximately 500, and they are partitioned into around 250 scolopidia. The mechanism of function has been studied extensively by Göpfert, Robert, Nadrowski and others $(25,77)$, mainly using laser Doppler vibrometry on the arista coupled with electrophysiology on the antennal nerve. In response to an external sound stimulus, the arista is deflected in such a way that it moves as one unit. This in turn produces a rotation in the funiculus (98). It has been hypothesized that when the funiculus rotates to one side, it compresses the sensory cilia of one of the opposing sets of neurons, and stretches those of the other set. These two sets 
would then be differentially stimulated, and the signal would be transduced into an electrical signal. A sound stimulus would manifest itself as a side-to-side oscillation of the arista, while a gravitational stimulus would result only in a deflection of the arista in one direction (25). Different groups of JO neurons have been shown to be involved in sound, wind and gravity sensing (99). There have also been extensive studies on the various mechanically activated ion channels (MACs) and other proteins involved in the process, and the effects of mutations in these proteins on JO function (82, 100-102). What has not yet been studied is how exactly the neurons and their cilia deform in response to a stimulus, and how this leads to opening of ion channels.

What makes $\mathrm{JO}$ especially interesting is that properties such as compressive non-linearity, frequency selectivity and spontaneous otoacoustic emissions, which have pointed towards an active amplification process in the vertebrate ear $(5,19,20,103)$, have also been observed for $\mathrm{JO}(25,77)$. Light microscopy, electron microscopy, genetics and molecular biology have provided detailed information about the structure, the identity and the function of various proteins that play a role in insect hearing and mechanosensing (12, 24, 25, 77, 82, 104, 105). Karak et al. (78) have investigated the role of the ciliary dyneins in the functioning of the JO. They describe two axonemal dynein genes - Dmdnah3 and Dmdnai2. Null alleles of both these genes result in absence of neural response to auditory stimuli. Mutations in the former promote active amplification, while mutations in the latter eliminate it.

The advantage of studying $\mathrm{JO}$ was that the sound receiver is external and can be easily monitored by laser Doppler vibrometry. However, the disadvantage is that there does not yet exist a method to directly probe the scolopidial, neuronal and ciliary mechanics and the responses of individual ion channels without damaging the mechanics of the antenna. The Ich5 organ (section 2.4) is a good candidate to study the mechanics of the scolopidial components, given that although it is not an auditory organ, it still functions using the same proteins as $\mathrm{JO}(44)$, and is more accessible to direct mechanical manipulation. 


\subsection{The Ich5 organ}

The Drosophila larva is a segmented animal (Fig. 2.4.1). Excluding the head, there are 10 segments -3 thoracic and 7 abdominal $(49,69)$. In each abdominal segment there are two lch5 organs, one each on the left and the right side. The scolopidia in an Ich5 organ are oriented parallel to each other (Fig. 2.4.2). The whole organ is about $300 \mu \mathrm{m}$ in length, and held taut by means of the attachment cells. The cap cells point dorsoposteriorly at an angle of approximately 45 degrees to the body axis (Fig. 2.4.3). The larval tissue is constructed in layers: The cuticle is the outermost layer, then comes the Ich5 organ, followed by 3 lateral muscles $(42,49,69)$ that are perpendicular to the body axis, followed by the dorsal and ventral muscles that are either parallel or at acute angles to the body axis (Fig. 2.4.3).

Ich5 organs are suspended between two attachment points at the cuticle, one on each side of the dorsal half of the larva in each segment in a tense straight conformation $(69,71,72)$. The Ich5 organs do not appear to be directly coupled to contractions of individual muscles. Rather, they sense deformations of the cuticle caused by muscle contraction - which is then transduced into a neural signal via the sensory neurons that are part of the organ (Halachmi et al., 2016 and Klein et al., 2010) $(71,72)$. A kink has been observed to form in the cap cells of Ich5 organ as its corresponding segment contracts during fictive crawling (106).

Zhang et al. describe an increase in calcium influx into the lch5 organ in response to vibration stimuli, and the roles of mechanosensory ion channels in this process (44). They claim that the organ responds to sound stimuli, and that this process is governed mainly by the NOMPC channel with downstream modulation by the channels NANCHUNG and INACTIVE. Their experiments involved placing larvae on agar in a petridish, and playing recordings of the sounds emitted by yellow jacket wasps - natural predators of the larvae - from a loudspeaker placed below. They coupled this technique with electrophysiology and calcium imaging. Whether or not the Ich5 organ is constructed to responds to sound stimuli is still debated, but it is still an interesting observation that the mechanically activated channels (MAC) and other proteins that play a major role in the lch5 organ are the same as those governing the functioning of Johnston's organ. 
In a study by Caldwell et al., mutations in chordotonal organs have been demonstrated to lead to locomotor defects in the larva, such as longer periods of time spent on turning and changing direction than on linear crawling in one direction (73). These results indicate that the chordotonal organs of the Drosophila larva provide feedback to the central nervous system to aid in muscle coordination necessary for crawling. The crawling mechanism is discussed in section 2.5.

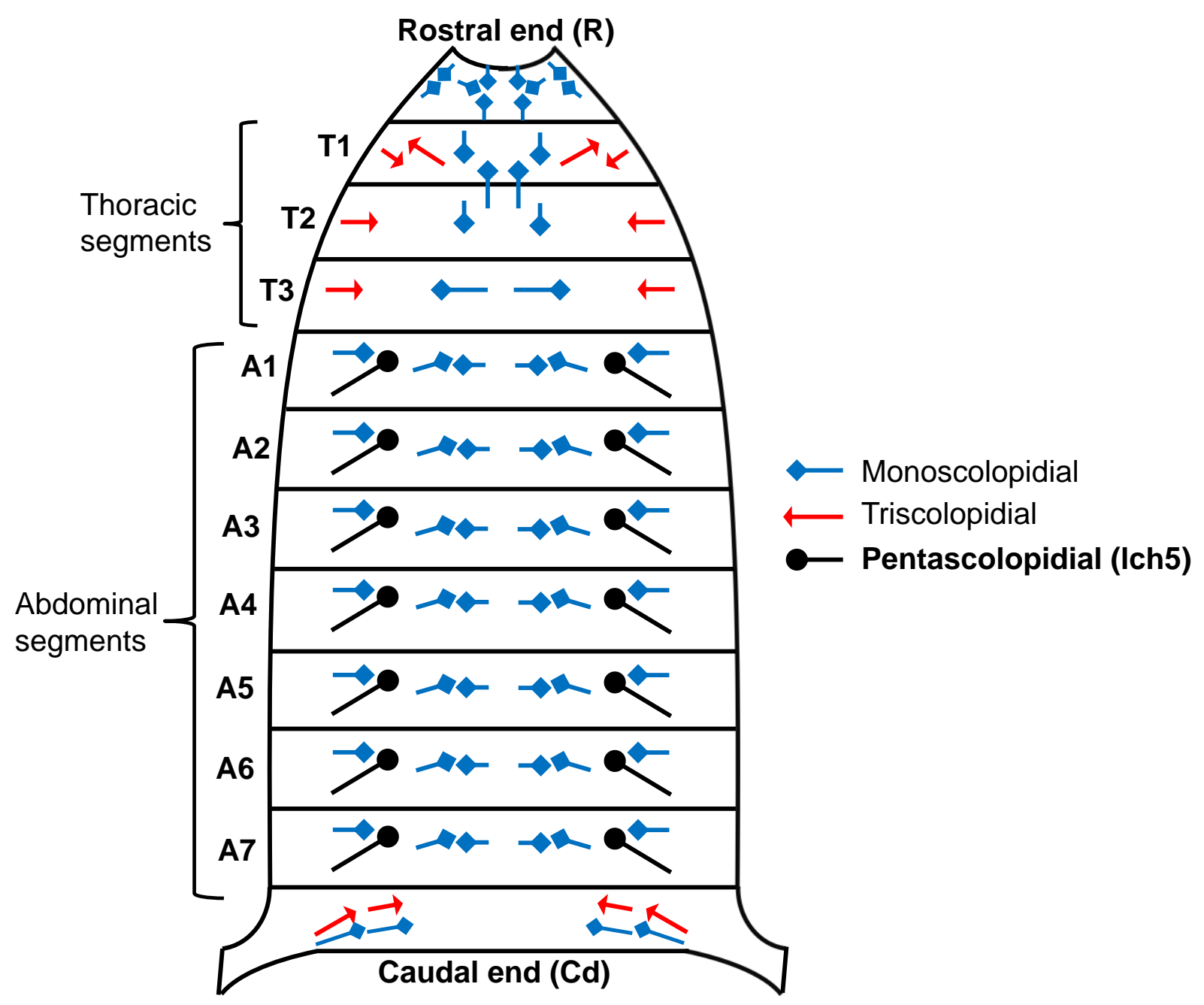

Fig. 2.4.1. Schematic representation of a fillet preparation (43) of a Drosophila larva, showing positions of various chordotonal organs including the Ich5 organ $(42,49)$. The heads of the arrows represent neuronal somata, and the tails represent cap cells. 


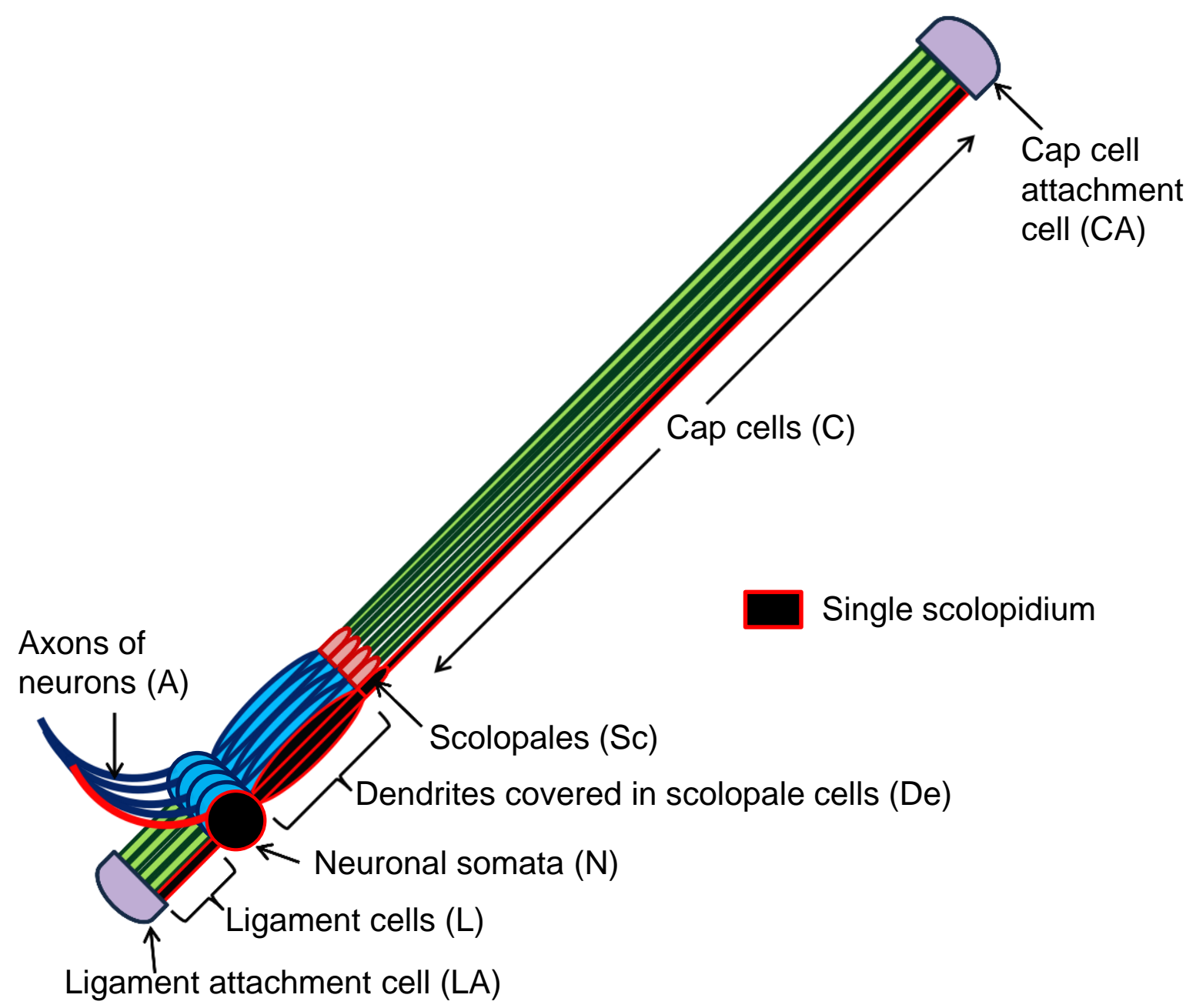

Fig. 2.4.2. Schematic of the Ich5 organ of the Drosophila larva $(49,69,72)$, depicting its various components. One of the 5 scolopidia has been darkened for easy identification. 


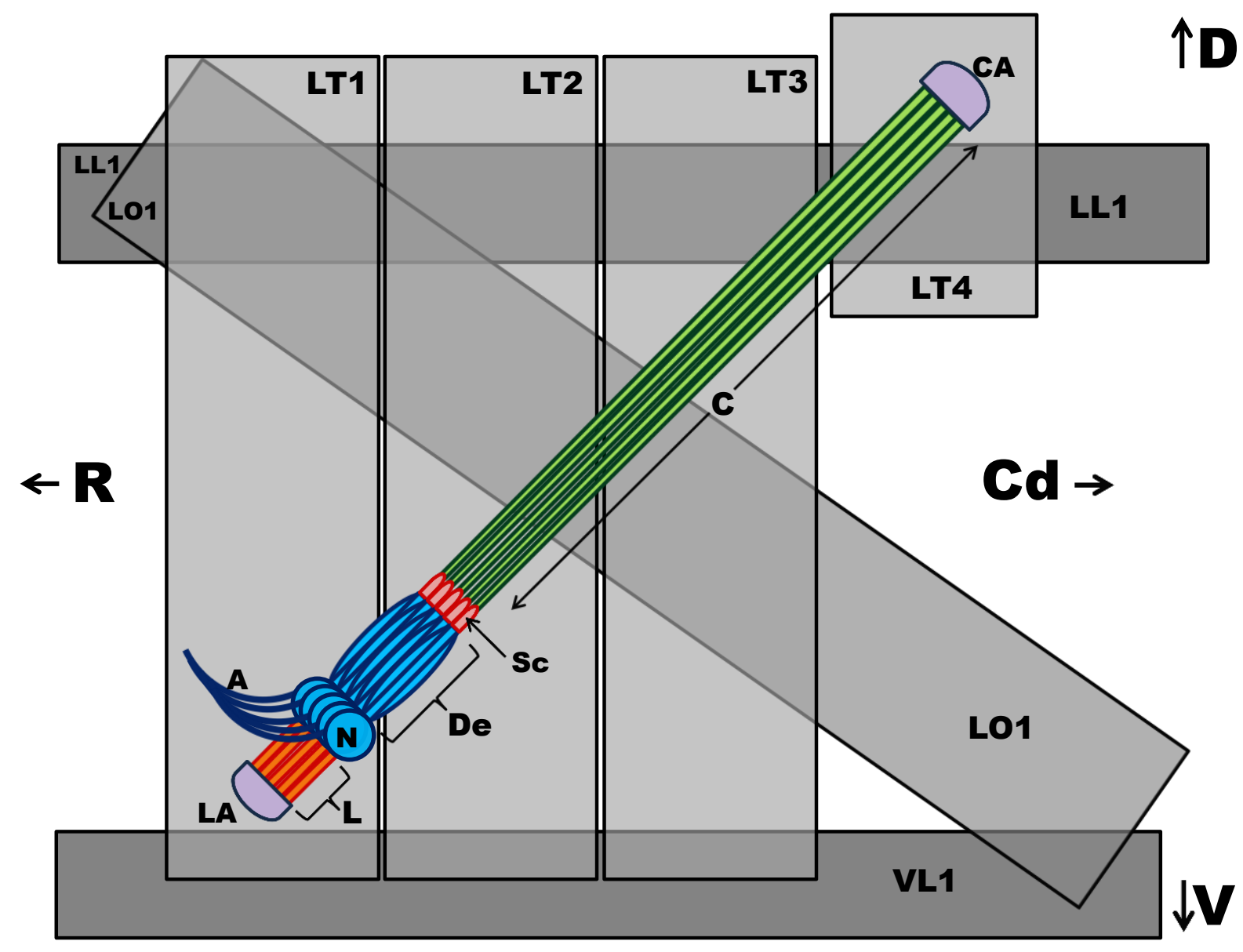

Fig. 2.4.3. Schematic of the Ich5 organ of the Drosophila larva $(49,69,72)$. Lateral view of a right hemisegment of the larva, oriented such that the rostral end $(R)$ of the larva is to the left and the caudal end (Cd) is to the right. LA: Ligament attachment cell, L: ligament cell, N: neuronal somata, D: dendrites, A: axons, Sc: scolopales, C: cap cell, CA: cap attachment cell. Rectangles indicate locations of the muscles that overlay the Ich5 organ towards the inside of the larva: Lateral transverse muscles LT1-4, lateral longitudinal muscle LL1, lateral oblique muscle LO1, ventral longitudinal muscle VL1 (there are 3 more VL muscles, as well as more oblique and LL muscles, which are not shown here). 


\subsection{Peristaltic crawling in the Drosophila larva}

The periodic peristaltic wave of body segments is the basis of crawling not only in the Drosophila larva, but also in many other soft-bodied animals such as earthworms, leeches and caterpillars. This wave can either travel in the same direction as the movement of the animal, or in the opposite direction. The former case applies to maggots such as the Drosophila melanogaster larva (38, 39, $42,73)$ and caterpillars such as Manduca sexta, while the latter applies to annelids such as earthworms. e.g. Lumbricus (107), and leeches, e.g. Hirudo medicinalis (108). Some propose that networks in the central nervous system, known as central pattern generators (CPG), are responsible for generating the oscillations that result in this periodic motion $(40,73,109,110)$. The CPG is said to accomplish this even in the absence of sensory input (111). Others suggest that the CPG may not be the dominant factor for locomotion in soft-bodied animals, and attribute a greater role to sensory feedback - such as Paoletti and Mahadevan (112), who in 2014 published a mathematical model for crawling locomotion that functions using not a CPG, but what they refer to as a "local feedback mechanism". This would involve interactions between the body of the animal and the surface on which it crawls, and local proprioception that presents feedback to the central nervous system. For the Drosophila larva, there does exist evidence of a CPG, but sensory feedback is of prime importance in coordinating the movement, as we shall see in the following paragraphs.

In Drosophila and Manduca, terms used to describe the crawling mechanism are "toothpasting" and "visceral piston". The visceral pistoning in the Drosophila larva has been described in great detail by Heckscher et al. in 2012 (42). These authors cite an earlier reference on Manduca crawling (113), and state that in order for a crawling mechanism to be called a visceral piston, it must have the following two features:

(a) The gut and the caudal end should move simultaneously as one unit, and

(b) The abdominal segments must not move during gut and caudal end motion.

They state that they have indeed observed such a mechanism. First the gut thrusts forward, and the rostral and caudal end move along with it. Then, a peristaltic wave begins at A7 and A8 (the seventh and eighth abdominal segments), and propagates forward, with two segments contracting at any given moment (42). Thus, there is no net increase or decrease of the length of the animal at any instant.

The Drosophila larva is capable of crawling in both forward and reverse directions $(42,73,109,114)$. There is a slight difference in the pattern of muscle contractions in these movements (42). In both cases, the dorsal oblique muscle 1 (DO1) and ventral longitudinal muscle 1 (VL1) contract before 
the lateral transverse (LT) muscles. In the case of the forward crawl, the DO1 and VL1 muscles contract simultaneously, followed by the LT muscles. In the other case, the DO1 muscle contracts with a slight delay as compared to the VL1 muscles, but still before the LT muscles.

Several studies show that the CPG of the Drosophila larva cannot function without feedback from mechanosensors. Hughes and Thomas stated in their 2007 paper that there is "effectively no independent CPG" in Drosophila larval crawling (115), meaning that sensory feedback is indispensable. A study by Caldwell et al. in 2003 (73) demonstrates that Drosophila larvae with chordotonal organ mutations have impaired locomotion, including phenotypes such as crawling in a zigzag fashion as opposed to linearly, difficulties in finding a suitable crawling direction, etc. Their results indicate that chordotonal organs, including our organ of interest - the lch5 organ, are involved in providing sensory feedback to the CPG for coordinated crawling. In close correlation with these observations are the findings of Suster and Bate, published in Nature in 2002 (109), which demonstrate that crawling-like patterns can be elicited in Drosophila embryos and larvae even if sensory feedback is completely eliminated, but in such a situation, the polarity of these patterns is disturbed and the reverse crawl predominates. Studies such as these have indeed proven that chordotonal organs are crucial to the crawling mechanism, but what remains to be seen is how the organs mechanically function. This would require direct physical probing of their viscoelastic properties. In this thesis we have undertaken to obtain an understanding of the Ich5 mechanics, as I describe in greater detail in the sections on experimental methods and results.

We used a dissected (fillet) preparation (43) of the larva that was immersed in Ringer's solution - a buffer solution similar in composition to the animal's haemolymph. To apply additional tension to the organ, we applied lateral force near the middle of the organ with a tungsten needle controlled by a micromanipulator. After rapid release of the needle, we recorded the free relaxation of the deformed organ to its initial conformation. From the stretched state, we could obtain estimates of the elastic deformation of the components of the Ich5 organ. We furthermore used UV laser ablation to sever the organ in different places to observe the elastic recoil of the different components of the organ. 


\subsection{Non-muscle myosin II in Drosophila}

There is only one type of non-muscle myosin II in Drosophila. Its heavy chain is encoded by a gene known as zipper $(116,117)$. The gene is so named because it plays a crucial role in the dorsal closure mechanism in the Drosophila embryo; mutations in this gene negatively impact not only dorsal closure but also head involution and patterning of axons (117). The basic structure of myosin II is hexameric, with two heavy chains and four light chains (118). In Drosophila non-muscle myosin II, one pair of light chains is known as cytoplasmic myosin light chain ( $m / c-c)$, and the other pair is known as regulatory spaghetti squash $(s q h)(116,118)$. In some of our experiments (laser ablation in the Ich5 organ), we have used a fly line where sqh has been labeled with GFP. This means that the ligaments and cap cells (section 2.4, and Fig. 2.1) are fluorescent when illuminated with blue light. Details are given in Materials and Methods (section 4.1) and Results and Discussion (section $5.6)$.

Non-muscle myosin II performs several functions in Drosophila. It is part of the actomyosin ring that contracts to repair wounds in the embryo (119). In the neuromuscular junction, it is present at the presynaptic terminal, and regulates the transport of synaptic vesicles (120). In the early embryo, it is involved in a barricading mechanism by which actomyosin actively blocks cells in one compartment from mingling with cells in other compartments (121). E-cadherin and actomyosin work together to produce polarized flows that drive morphogenesis (122). Myosin II has central roles in several types of cell deformation operating in development (123).

In chordotonal organs such as the Ich5 organ, non-muscle myosin II is present in the cap cells and the ligaments in greater quantity than in the neurons (124-126). Also, the scolopales are said to contain actin but not myosin (124-126). Our results point to an important role of myosin II in the mechanics of the Ich5 organ. I describe these in sections 4.5 and 4.6. 


\section{Materials and methods}

\subsection{List of chemicals}

\begin{tabular}{|c|c|c|}
\hline Chemical & Manufacturer or source & Catalog number \\
\hline $\begin{array}{l}\text { Sylgard } 184 \text { silicone elastomer } \\
\text { kit - consisting of elastomer } \\
\text { and curing agent }\end{array}$ & $\begin{array}{l}\text { Dow Corning, Wiesbaden, } \\
\text { Germany }\end{array}$ & 000000000001064291 \\
\hline $\mathrm{NaCl}$ & Merck, Darmstadt, Germany & 106404 \\
\hline $\mathrm{KCl}$ & Carl Roth, Karlsruhe, Germany & 6781 \\
\hline $\mathrm{NaHCO}_{3}$ & $\begin{array}{l}\text { Honeywell Riedel de Haën, } \\
\text { Seelze, Germany }\end{array}$ & 000000015541 \\
\hline Trehalose & Sigma-Aldrich, Munich, Germany & T9531 \\
\hline Sucrose & AppliChem & A2211 \\
\hline $\mathrm{MgCl}_{2}$ & Carl Roth, Karlsruhe, Germany & KK36 \\
\hline $\mathrm{CaCl}_{2}$ & Carl Roth, Karlsruhe, Germany & CN93 \\
\hline HEPES & Carl Roth, Karlsruhe, Germany & HN77 \\
\hline $\begin{array}{l}\text { Oil for objectives: } \\
\text { Immersol }^{\mathrm{TM}} 518 \mathrm{~F}\end{array}$ & $\begin{array}{l}\text { Carl Zeiss, Oberkochen, } \\
\text { Germany }\end{array}$ & $12-624-66 \mathrm{~A}$ \\
\hline Fly food & $\begin{array}{l}\text { Prepared at the lab of Prof. Dr. } \\
\text { Martin Göpfert, Schwann- } \\
\text { Schleiden Research Center, } \\
\text { University of Göttingen, } \\
\text { Germany. }\end{array}$ & Not applicable \\
\hline Agarose & Carl Roth, Karlsruhe, Germany & 2267 \\
\hline Collagenase & Sigma-Aldrich, Munich, Germany & C0130 \\
\hline Superglue & $\begin{array}{l}\text { UHU GmbH \& Co, Bühl (Baden), } \\
\text { Germany }\end{array}$ & 4026700455700 \\
\hline Epoxy glue & Liqui-Moly GmbH, Ulm, Germany & 6183 \\
\hline
\end{tabular}




\subsection{Maintenance of fly stocks:}

All flies were bred on food prepared at the laboratory of Prof. Dr. Martin Göpfert, Schwann-Schleiden Research Center, University of Göttingen, Germany. They were maintained in plastic vials that were quarter-filled with food, and stored at a temperature of $25^{\circ} \mathrm{C}$ or $18^{\circ} \mathrm{C}$. The food was prepared using the following standard protocol (81):

$102 \mathrm{~g}$ agar was soaked overnight in $5 \mathrm{~L}$ tap water. The following mixtures were prepared:

1. $100 \mathrm{~g}$ soybean flour and $180 \mathrm{~g}$ yeast in $1 \mathrm{~L}$ tap water

2. $800 \mathrm{~g}$ cornmeal in $2 \mathrm{~L}$ water

3. $220 \mathrm{~g}$ treacle in $1 \mathrm{~L}$ water

The above mixtures were boiled together at $100^{\circ} \mathrm{C}$, in the Varioklav $\AA$ Steampot DT44580604 machine. Once the mixture cooled to $55^{\circ} \mathrm{C}$, the two following mixtures were added to it:

1. $800 \mathrm{~g}$ malzin in $1 \mathrm{~L}$ water

2. $62 \mathrm{~mL}$ propionic acid and $150 \mathrm{~g}$ nipagin in $80 \mathrm{~mL}$ ethanol

Using an Isomatic MCP pump, the warm food was filled into plastic vials. Once it cooled down, it congealed. The vials were closed with mite-proof sponge plugs, and stored at $4^{\circ} \mathrm{C}$ for up to 4 weeks. If they were used immediately, they could also be stored at room temperature. 


\subsection{Fly lines used:}

\begin{tabular}{|c|c|}
\hline Experiment & Fly line \\
\hline Crawling assays (3.5) & elaV-GCaMP \\
\hline $\begin{array}{l}\text { DIC imaging of the Ich5 organ (3.7) } \\
\text { Relaxation dynamics/ shape analysis (3.8) } \\
\text { Measurement of force using calibrated glass } \\
\text { pipettes (3.10) }\end{array}$ & Wild-type: Canton-S (CS), OregonR (OrR) \\
\hline Laser ablation (3.9) & $\begin{array}{l}\text { 1. OrR, CS (wild type) } \\
\text { 2. w[-];PBac[20XUAS- } \\
\text { 6XGFP]VK00018;iav-GAL4,w[+]/+: } \\
\text { Exhibits GFP fluorescence in Ich5 } \\
\text { neurons (axons, somata, dendrites, } \\
\text { scolopales). These animals are } \\
\text { heterozygous, and therefore need to be } \\
\text { checked for fluorescence prior to use. } \\
\text { This was done using a Leica MZ FLIII } \\
\text { microscope, with the appropriate filter } \\
\text { for GFP fluorescence. } \\
\text { 3. Sqh-GFP: In this line, non-muscle } \\
\text { myosin has been labeled with GFP. } \\
\text { Hence it exhibits GFP fluorescence in } \\
\text { the cap cells and ligaments but not in } \\
\text { the neurons. } \\
\text { 4. UAS Sqh-RNAi X Pinta-GAL4 (myosin } \\
\text { knockdown mutant): In this mutant, the } \\
\text { non-muscle myosin is partially knocked } \\
\text { down in the chordotonal organs, } \\
\text { however not completely eliminated. } \\
\text { w }{ }^{118} X \text { Pinta-GAL4: This was used as a } \\
\text { control for the myosin mutant larvae. }\end{array}$ \\
\hline
\end{tabular}




\subsection{Optical trapping}

Optical trapping is a technique by which a finely focussed laser is used to hold an object at its focal point by optical forces $(127,128)$. One may either use an optical trap to measure forces, or to deliver known forces. Earlier trapping techniques used two lasers, or depended on gravity. But later on, the single-beam gradient trap, also known as optical tweezers, was designed (127). This makes use of a single laser beam, and only optical forces are used to trap the object.

The single-beam optical trap was originally designed to trap particles in the Rayleigh range, i.e. of size smaller than the wavelength $\lambda$ of the laser (128). Examples of such particles could be atoms and molecules. In this case, the particle can be represented as a dipole (127). Then there are two forces acting on the particle, which shall be now described. When laser light is focused into a diffraction-limited focus, the light intensity strongly increases as one moves closer to the focus. The force arising from this intensity gradient is known as the gradient force. The other force points in the direction of the incident light, and is known as the scattering force (127). (Ashkin 1992, Gordon 1973, Gordon and Ashkin 1980). The gradient force pulls the particle into the laser focus, while the scattering force tends to eject it from the trap. More details of these forces can be found in (129) and .

For a Rayleigh particle, trapping is achieved when the gradient force is stronger than the scattering force. It was later discovered that even particles whose sizes are in the micron range can be trapped by a laser (130). These could be silica (27) or polystyrene beads (131), or even biological samples such as living cells (eukaryotic cells, bacteria, viruses) (132). In biological optical trapping experiments, one typically traps dielectric beads and uses them as probes, or traps whole cells. 


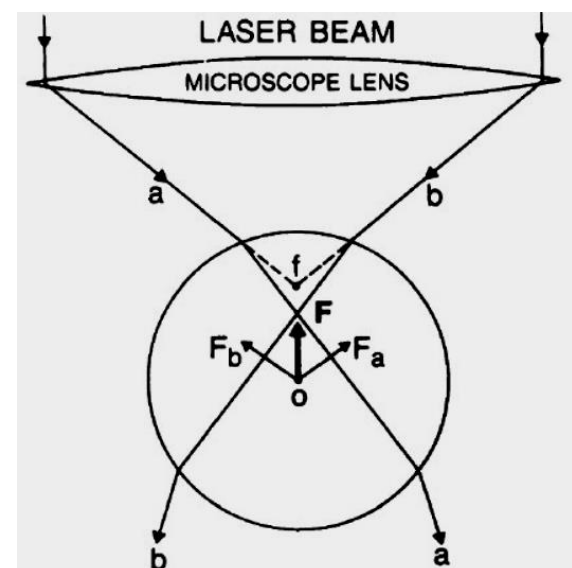

A

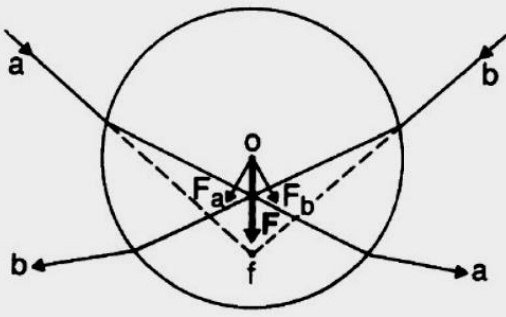

B

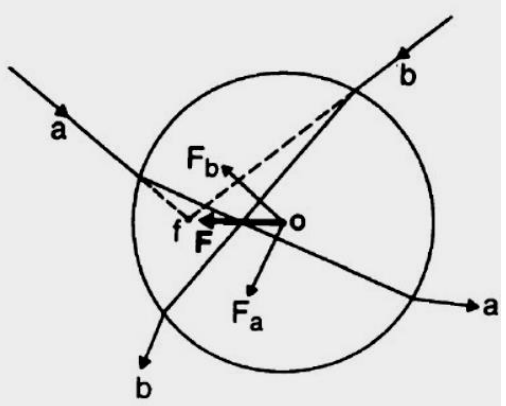

C

Fig. 3.4.1. A diagram of an optically-trapped bead, showing the direction of gradient force for different positions of the laser focus. Image modified from the following sources: (133) and (127).

In biophysical experiments, optical trapping is generally used to measure forces. This force could be exerted by the flagellum of a bacterium (134) or a sperm cell as it swims (135). Other examples are the forces exerted by migrating cells in a developing Drosophila embryo (133), or by a kinesin molecule walking on the surface of a microtubule (136). Alternatively, one could use an optically trapped probe such as a polystyrene bead to deliver forces to the system, and measure the response say by calcium imaging or electrophysiology. Forces delivered are typically of the order of a few $\mathrm{pN}$ (129). An example of such an experiment is to displace the non-motile primary cilia of a Madin-Darby Canine Kidney (MDCK) cell with an optically trapped bead, and measure the calcium influx (27). Another such experiment involves two optical traps with beads in them placed at either end of a cell (131). This dual optical trap has been used to measure viscoelastic properties of fibroblasts and cardiomyocytes (131).

Results from optical trapping experiments can be presented as a plot of power spectral density versus frequency $(27,137)$. For a dielectric bead trapped at a laser focus in an aqueous medium, the power spectral density is the square of the Fourier transform of the Brownian fluctuations of the bead.

It is important to note that infrared lasers are preferred for biological optical trapping experiments, since they cause the least radiation damage (138). However, even this may be too intense for a highly absorbing system like the arista of Drosophila flies (see section 4.1). 


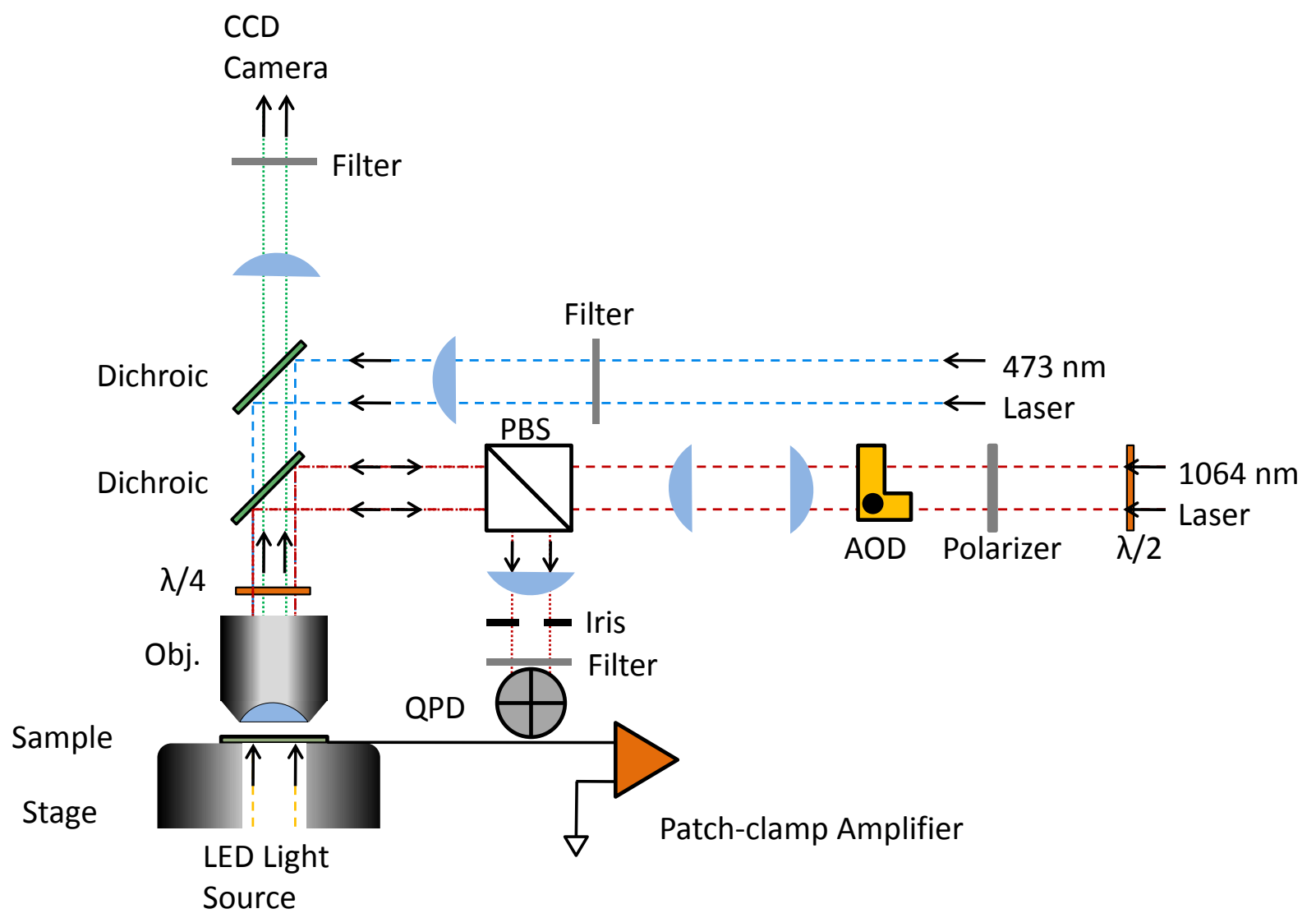

Fig. 3.4.2. The set-up used for my optical trapping experiments. Figure courtesy Dr. Christopher Battle.

My experiments were carried out on an optical tweezer set-up that had been built and aligned by Dr. Christopher Battle (27) while he was working at the group of Prof. Dr. Schmidt (Fig. 3.4.2). The set-up consists of an upright microscope. I used the following objectives: W Plan-Apochromat 40x/1.0 DIC (Carl Zeiss AG, Göttingen, Germany) and LUMPlanFI 60x/1.0 water-dipping objectives (Olympus Europa SE \& Co. KG, Hamburg, Germany). Videos were recorded using a CCD camera (Photometrics Coolsnap EZ, Roper Scientific GmbH, Göttingen, Germany).

\subsubsection{Experiments on adult flies}

The trapping experiments were carried out as follows:

The head of an adult fly (or in some cases an entire fly) was fixed at the bottom of a petridish using superglue. The arista was located using the microscope. The petridish was filled with water, and polystyrene or silica beads $(4 \mu \mathrm{m})$ were injected using a pipette. The IR laser (an ytterbium fiber laser, 1064 nm, from IPG Laser $\mathrm{GmbH}$, Burbach, Germany) was switched on and a bead was trapped at its focus. This bead was then moved close to the arista. 


\subsubsection{Experiments on larvae}

Here we used a fillet preparation of the larva, described in detail in section 3.8.4. After locating an Ich5 organ, I injected beads into the Ringer solution using a pipette. Muscles presented an obstacle to measurements. Hence, I used an apparatus made of a glass pipette filled with concentrated collagenase solution, attached at the opposite end to a rubber tube and a plastic pipette, to blow collagenase onto the muscles. This pipette was attached to a micromanipulator (Scientifica UI-1000-i, Multichannel Systems, Reutlingen, Germany). I also used the same pipette to tear away the remnants of the muscles. Once the Ich5 organ was free of muscles, I injected beads into the Ringer solution. When a bead came in contact with the Ich5 organ, I switched on the laser and attempted to trap it. Since the desired effect was not observed, we changed our methodology, as is described in subsequent sections, and in more detail in Results and Discussion. 


\subsection{Crawling assay}

A 30\% agarose gel was prepared by dissolving agarose in a glass flask in a microwave oven. Then a glass slide was taken, coated with the agarose solution and allowed to cool. Once the gel formed, a groove was made in it, in which a third instar larva was placed and allowed to crawl. Images were recorded using a Zeiss Observer Z.1 microscope and an Andor Zyla camera. The following objectives were used: ACHROPLAN 10x/0.25 and LD ACHROPLAN 20x/0.40 (Carl Zeiss AG, Göttingen, Germany). The set-up is shown in Figs. 3.5.1 and 3.5.2.

The gels had to be freshly prepared each time, since they dry out within a few hours.

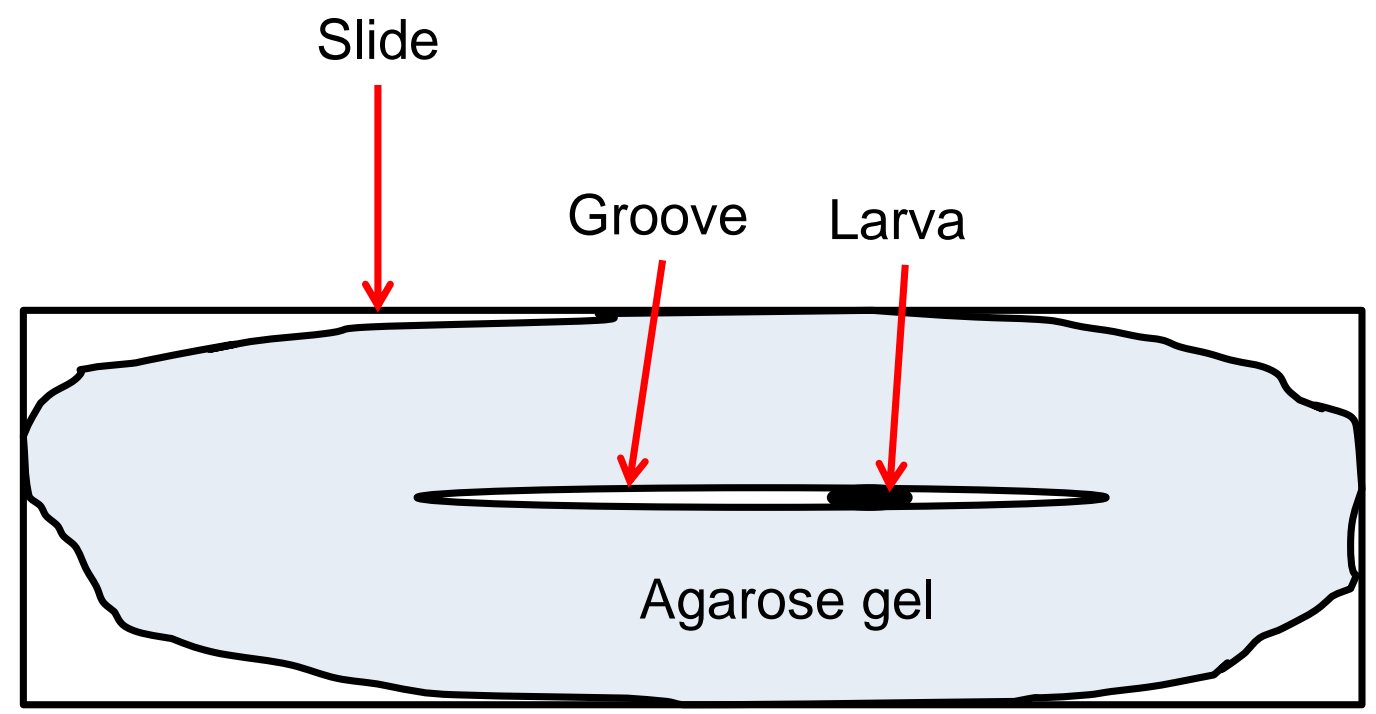

Fig. 3.5.1. Agarose-coated slide used for crawling assay 


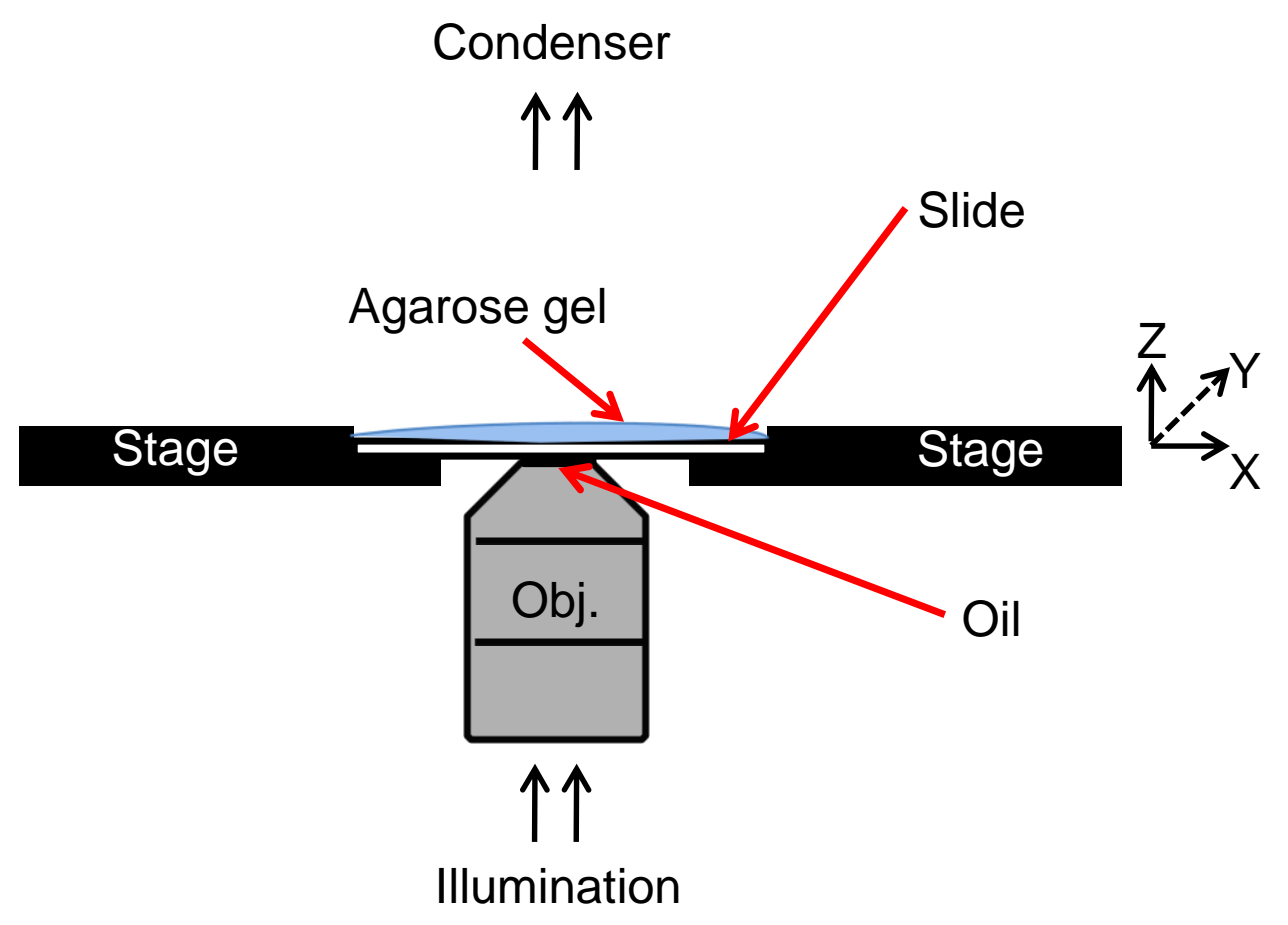

Fig. 3.5.2. Set-up used for crawling assay, showing relative placement of components

\subsection{The "squished prep"}

This preparation was used for DIC imaging of the Ich5 organ (section 3.7 in Materials and Methods, and section 4.3 in Results) and laser ablation experiments (section 3.9 in Materials and Methods, and section 4.6 in Results).

This method of preparing the larva sample was developed as follows: In order to obtain detailed images of the components of the lch5 organ, described in section 2.4 in Scientific Background, transillumination DIC microscopy is the method of choice, since it yields images with sufficient contrast. DIC microscopy requires thin and transparent samples. Mounting the fillet preparation (section 3.8.4) on a slide was not feasible, since the fillet was observed to get damaged when detached from the PDMS slab. Hence, I devised the preparation described below. This so-called "squished prep" has proved highly useful also in the laser ablation experiments.

Before carrying out the experiment, the larvae were squeezed between a glass slide and a coverslip along with some Ringer's solution, such that the gut was removed and the interior of the larva became transparent (Fig. 3.6.1). This method leaves the chordotonal organs well-preserved and 
clearly visible, albeit not accessible to direct mechanical manipulation. One slide and coverslip can accommodate several larvae.

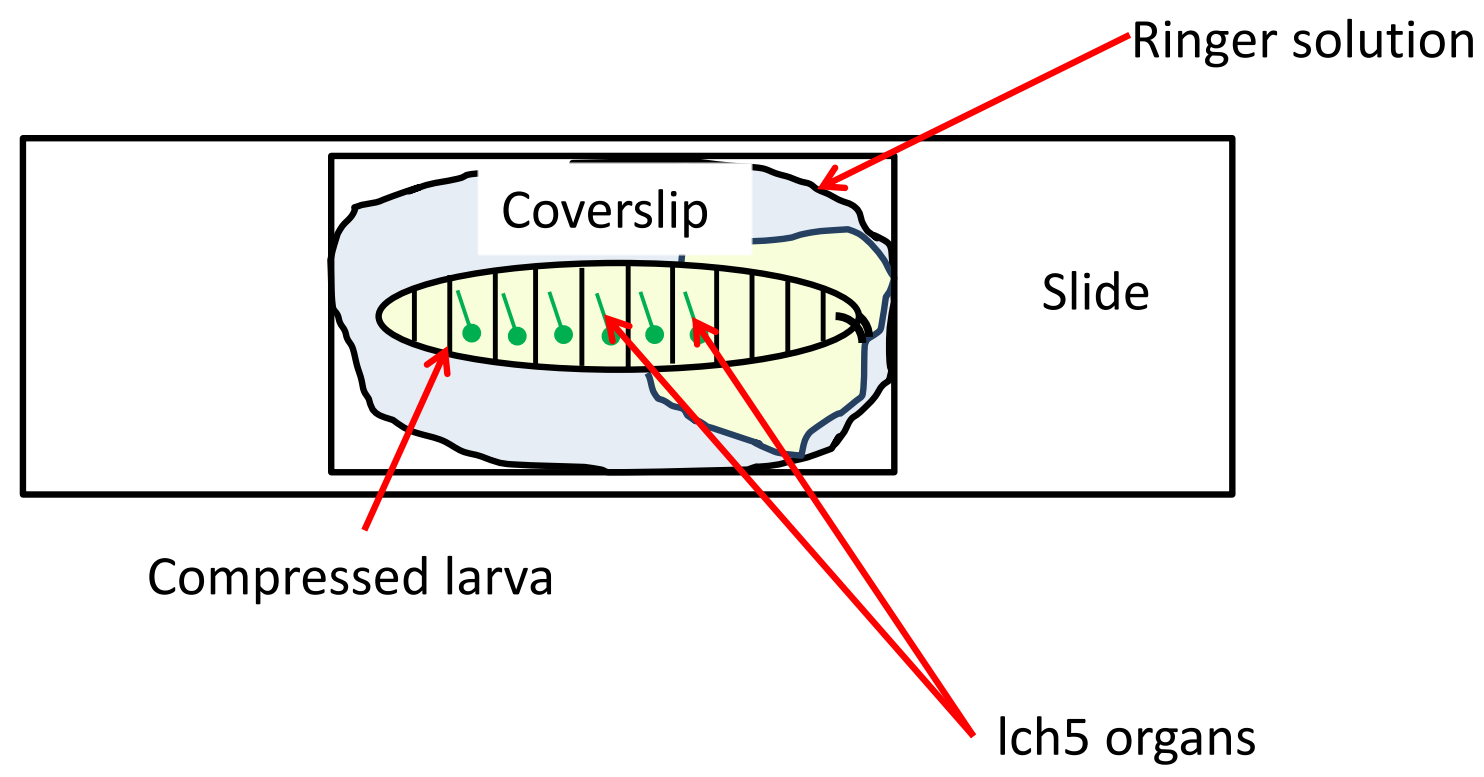

Not to scale

Fig. 3.6.1. Depiction of the larval preparation used for the DIC imaging and the UV laser cutting experiments. In this diagram, the larva has been enlarged several times from its actual size relative to that of the coverslip, in order to make its placement clear. The larva was not always placed parallel to the long axis of the slide as shown. It could be at any angle.

\subsection{DIC images of the Ich5 organ}

The larva was prepared using the "squished prep" method as described in the previous section. A Zeiss Axiovert 200 microscope was used, with the DIC-II prism. The sample was viewed using a Plan-APOCHROMAT 100x/1.46 objective (Carl Zeiss AG, Göttingen, Germany). A drop of oil was placed on the objective, and the sample was placed on the microscope. An lch5 organ was located, and the stage was gradually moved from one end of the organ to the other, intermittently recording and saving snapshots using a CCD camera (Photometrics Coolsnap EZ, Roper Scientific GmbH, Göttingen, Germany) and the WinSpec/32 software (Princeton Instruments, New Jersey, USA). 


\subsection{Relaxation kinetics and shape analysis}

\subsubsection{Preparation of Ringer buffer}

To prepare Ringer's solution, the following ingredients were dissolved in high purity Millipore water $\mathrm{NaCl} 0.07 \mathrm{M}, \mathrm{KCl} 5 \mathrm{mM}, \mathrm{NaHCO}_{3} 0.1 \mathrm{M}$, trehalose $5 \mathrm{mM}$, sucrose $0.115 \mathrm{M}, \mathrm{CaCl}_{2} 2 \mathrm{mM}, \mathrm{MgCl}_{2} 0.02$ M, HEPES $4.2 \mathrm{mM}$. 1L solution was prepared in a glass bottle, and was constantly stirred using a magnetic stirrer till the contents dissolved. The $\mathrm{pH}$ was adjusted to 7.2. The Ringer's solution was poured into 20 Falcon tubes each of $50 \mathrm{~mL}$ capacity, stored frozen, and thawed before use. It was important to freeze the solution, because if it was stored at room temperature or even in a refrigerator for a very long time, the presence of large amounts of sucrose in the solution would lead to contaminations.

\subsubsection{Preparation of PDMS slabs}

Elastomer and curing agent (Sylgard 184 silicone elastomer kit) were thoroughly mixed at a ratio of 10:1 (4 mL elastomer, $0.4 \mathrm{~mL}$ curing agent) in a borosilicate glass beaker of $30 \mathrm{~mm}$ inner diameter and baked for $10 \mathrm{~min}$ in an oven preheated to $150^{\circ} \mathrm{C}$, followed by freezing at $-20^{\circ} \mathrm{C}$ for 5 min to help detach the PDMS from the beaker walls. Through adhesion to the beaker walls before polymerization, the PDMS slabs developed slightly elevated edges that helped to retain the buffer solution under the microscope. The slabs were carefully extricated from the beakers using a scalpel. The method was modified from the following source: http://www.digitaladdis.com/sk/PDMS Mold Preparation Kassegne MEMSLab.pdf (accessed May 7, 2016).

\subsubsection{Preparation of tungsten needles}

Tungsten wire (0.2 mm diameter) was purchased from Goodfellow GmbH, Bad Nauheim, Germany. Fine-tipped tungsten needles were produced by electrolytically sharpening pieces of this wire on a machine custom-designed for the purpose by Dr Bart Geurten in the group of Prof. Dr. Martin Göpfert (Fig. 3.8.1 A). The sharpening was carried out by repeatedly dipping the wire into concentrated $\mathrm{KOH}$ solution (2M) using a rotary mechanism, while pausing the procedure intermittently to check the needle for sharpness via a microscope. 
The two components of the epoxy glue were mixed, and the blunt end of the needle was immersed in it. Then the blunt end of the needle was affixed to a glass capillary, and left for 5 minutes while the glue hardened. Then the tip of the needle was bent at an angle of approximately $90^{\circ}$ for a length of about $2 \mathrm{~mm}$ (Fig. 3.8.1 B), so that it could be hooked into the Ich5 organ vertically. This was done as follows: The portion of the needle that protruded from the capillary was inserted into another capillary, and bent by moving the second capillary, taking care not to damage the sharp tip.

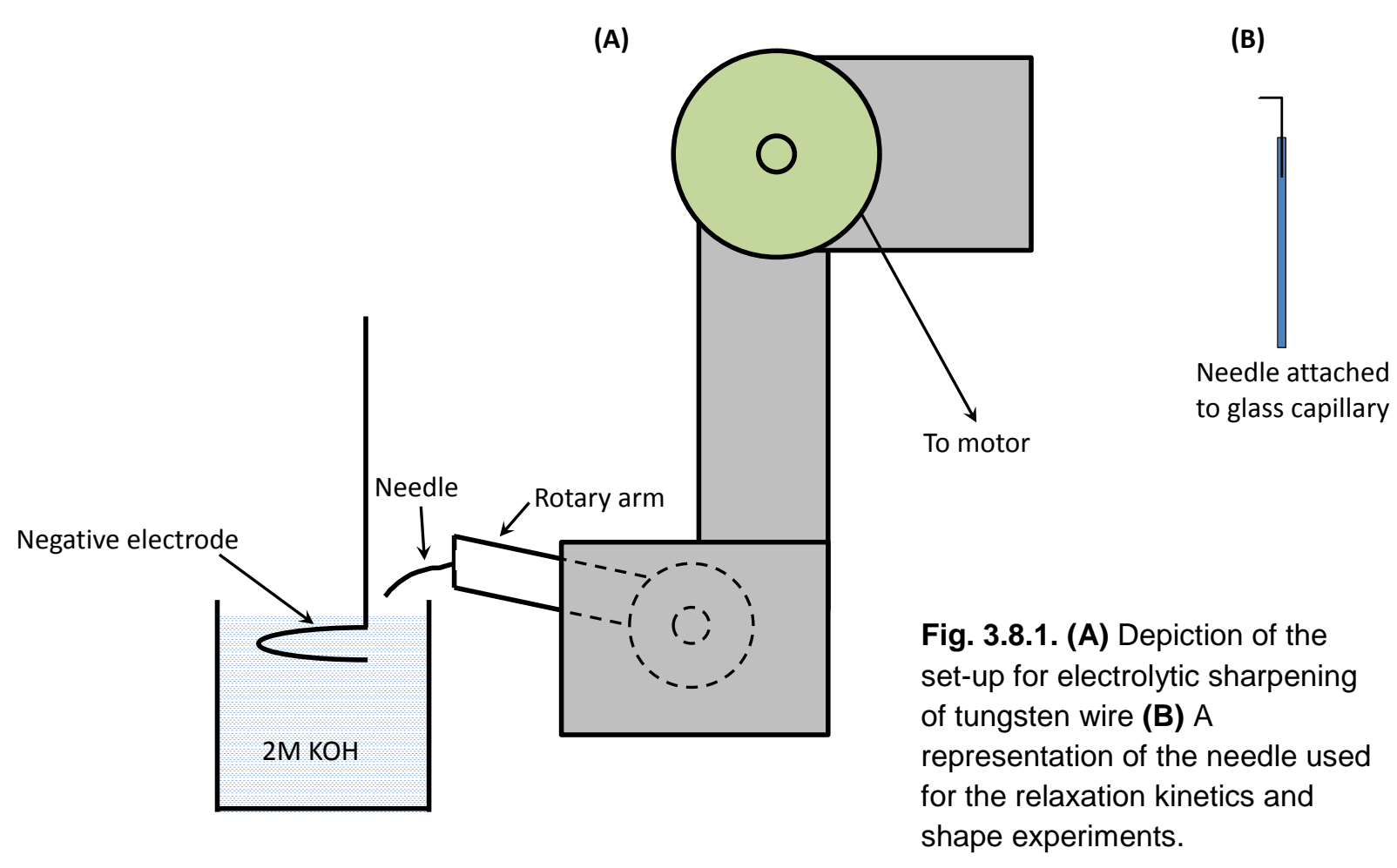


3.8.4. Preparation and dissection of Drosophila larvae

\begin{tabular}{|c|c|c|}
\hline Tool & Application & $\begin{array}{l}\text { Catalog number } \\
\text { (if applicable) }\end{array}$ \\
\hline $\begin{array}{l}\text { Dissection pins: Pieces of steel } \\
\text { wire of } 0.1 \mathrm{~mm} \text { diameter. These } \\
\text { were sourced from the machine } \\
\text { workshop at the DPI, Faculty of } \\
\text { Physics, University of } \\
\text { Göttingen. } \\
\text { (OR) } \\
\text { Austerlitz Insect Pins } ®, 0.1 \mathrm{~mm}\end{array}$ & $\begin{array}{l}\text { To pin the larva in place during } \\
\text { dissection }\end{array}$ & $\begin{array}{l}\text { Steel wire: not applicable } \\
\text { Insect pins: catalog } \\
\text { number } 26002-10\end{array}$ \\
\hline DuMont \#55 Forceps & $\begin{array}{l}\text { Holding the dissection pins: for } \\
\text { this, old forceps that had become } \\
\text { blunt were used (new forceps } \\
\text { were reserved for gut removal, } \\
\text { since using them for pin holding } \\
\text { would damage them) } \\
\text { Pulling out the gut of the larva } \\
\text { fillet: for this I used new, sharp } \\
\text { forceps. }\end{array}$ & $11295-51$ \\
\hline Ultra-Fine Clipper Scissors & $\begin{array}{l}\text { To cut open the larva along its } \\
\text { midline }\end{array}$ & $15300-00$ \\
\hline Vannas Spring Scissors & $\begin{array}{l}\text { To cut away the muscles of the } \\
\text { larva }\end{array}$ & $15000-04$ \\
\hline
\end{tabular}

Table 3.8.1. The various dissection tools, and their uses. All tools were sourced from Fine Science Tools (FST), Heidelberg, Germany, unless otherwise mentioned in the table.

I used third instar CS or OrR wild type larvae for all experiments and essentially followed published procedures for dissection (43). Dissection was carried out on circular PDMS slabs of $30 \mathrm{~mm}$ diameter and $\sim 4 \mathrm{~mm}$ edge height with a shallow central depression of $\sim 2 \mathrm{~mm}$, which were prepared as 
described in section 3.8.2. The dissected larva thus prepared is known as a fillet (43). The dissection procedure was as follows:

1. The PDMS slab was placed in a plastic petri dish of $10 \mathrm{~cm}$ diameter, and pressed down so that its bottom adhered to the petri dish. Then the following steps were carried out using a stereomicroscope (Leica M80, Leica Microsystems, Wetzlar, Germany) at 2.5X magnification.

2. The larva was pinned at its caudal end, approximately in the middle of the PDMS slab.

3. Next, the larva was held taut - but not stretched excessively - and pinned at its rostral end.

4. Ringer solution was added with a plastic dropper till the PDMS slab was filled and the larva was completely submerged.

5. Using the Ultra-Fine Clipper Scissors, a cut was made close to the caudal end. The gut was seen to float upwards. It was partly pulled out, and then the cut was extended till it reached the rostral end. Ringer solution was taken out of the PDMS slab and sprayed back on the sample, till the gut was partly disengaged from the cuticle and rendered easy to remove.

6. Using sharp forceps, the gut was gently pulled out, taking care not to damage the fillet.

7. 4 more pins were used to open out the fillet and make it flat (Fig. 3.8.2).

8. The preparation (fillet) was washed 2-3 times using Ringer's solution.

9. The larvae were then kept under fresh Ringer's solution and used in the experiments within $10 \mathrm{~min}$ after beginning the preparation and for not longer than $2 \mathrm{~h}$.

In some preparations, I additionally excised muscles using Vannas Spring Scissors. This was done to expose the lch5 organs and compare the relaxation properties to the case where the muscles were intact. The same stereo-microscope was used, and the excision was done at 6X magnification. This method was learnt from Dr Chonglin Guan in the group of Prof. Dr. Christoph F. Schmidt, Third Institute of Physics - Biophysics, Faculty of Physics, University of Göttingen. 


\subsubsection{Imaging and mechanical manipulation}

The fillet preparation was viewed using a 20X water-dipping objective (W Plan-Apochromat 20x/NA 1.0, Carl Zeiss Microscopy AG, Göttingen, Germany) in the upright microscope on the set-up described in section 3.4 that had been originally designed for optical trapping experiments (27). For the purpose of these experiments, we replaced the CCD camera, which could only achieve a maximum frame rate of $20 \mathrm{fps}$, with a high-speed camera (Photron Fastcam, VKT Video Kommunikation $\mathrm{GmbH}$, Pfullingen, Germany). The sample was placed on a stage that could be moved along $X, Y$ and $Z$ axes, from Newport Corporation, Irvine, USA. The following software was used for imaging: MicroManager (https://micro-manager.org) (139) which works in combination with ImageJ (https://imagej.nih.gov/ij/) (140).

First, the meniscus of the Ringer solution layer on the fillet was brought in contact with the objective. Then the larva sample was observed, and moved into focus such that an lch5 organ was visible. Then the sample was rotated such that the lch5 organ was oriented roughly parallel to the long axis of the image window of MicroManager (Fig. 3.8.2, lower part, also Fig. 4.5.1 in Results). After focusing on the Ich5 organ, the sample was lowered. Then, using a micromanipulator (Scientifica UI1000-i, Multichannel Systems, Reutlingen, Germany), the tungsten needle was brought into focus (in air). The sample stage was raised and the lch5 organ was focused on once again. The needle, which had by now gone out of focus, was lowered to bring it back in focus and in contact with the Ich5 organ. Once this was done, a lateral pushing or a pulling force was exerted at an angle of $90^{\circ}$ to the Ich5 organ, to deform and stretch the organ. The needle was then rapidly disengaged using the "step" function of the manipulator. Videos were recorded at frame rates of 250 and 1000 fps. For the larval preparations with intact muscles, the fast time constant was resolved equally well at both frame rates. For the preparations with muscles excised, a frame rate of $1000 \mathrm{fps}$ was used, since the fast time constant was smaller than in the previous case. Details are given in section 4.4. The videos were analyzed using the Manual Tracking plugin on ImageJ (https://imagej.nih.gov/ij/plugins/track/track.html), either by tracking several points along the organ or by specifically tracking the motion of the distinctive scolopales.

In the preparations with intact muscles, four Ich5 organs, each from a different animal, were tested (Animal 1: 2 trials - A1 \& A2, Animal 2: 3 trials each from 2 different organs - B1-B3 and C1-C3, Animal 3: 5 trials - D1-D5, Animal 4: 5 trials - E1-E5). The results from these trials are depicted in Figs. 4.4.4 - 4.4.5 and Table 4.4.1. For the case with excised muscles, three trials each were performed on two organs from two different animals (Animal 1: W1 - W3, Animal 2: X1 - X3). The results from these trials are depicted in Figs. 4.4.4 - 4.4.5 and Table 4.4.2. The position data obtained from ImageJ was fitted by single or double exponentials in OriginPro 
(http://www.originlab.com/Origin). Some further videos were recorded at $20 \mathrm{fps}$, using the CCD camera. These were used to analyze the shape change of the organ as it was deformed by the needle and then released. The set-up is depicted in Figs. 3.8.2 and 3.8.3.

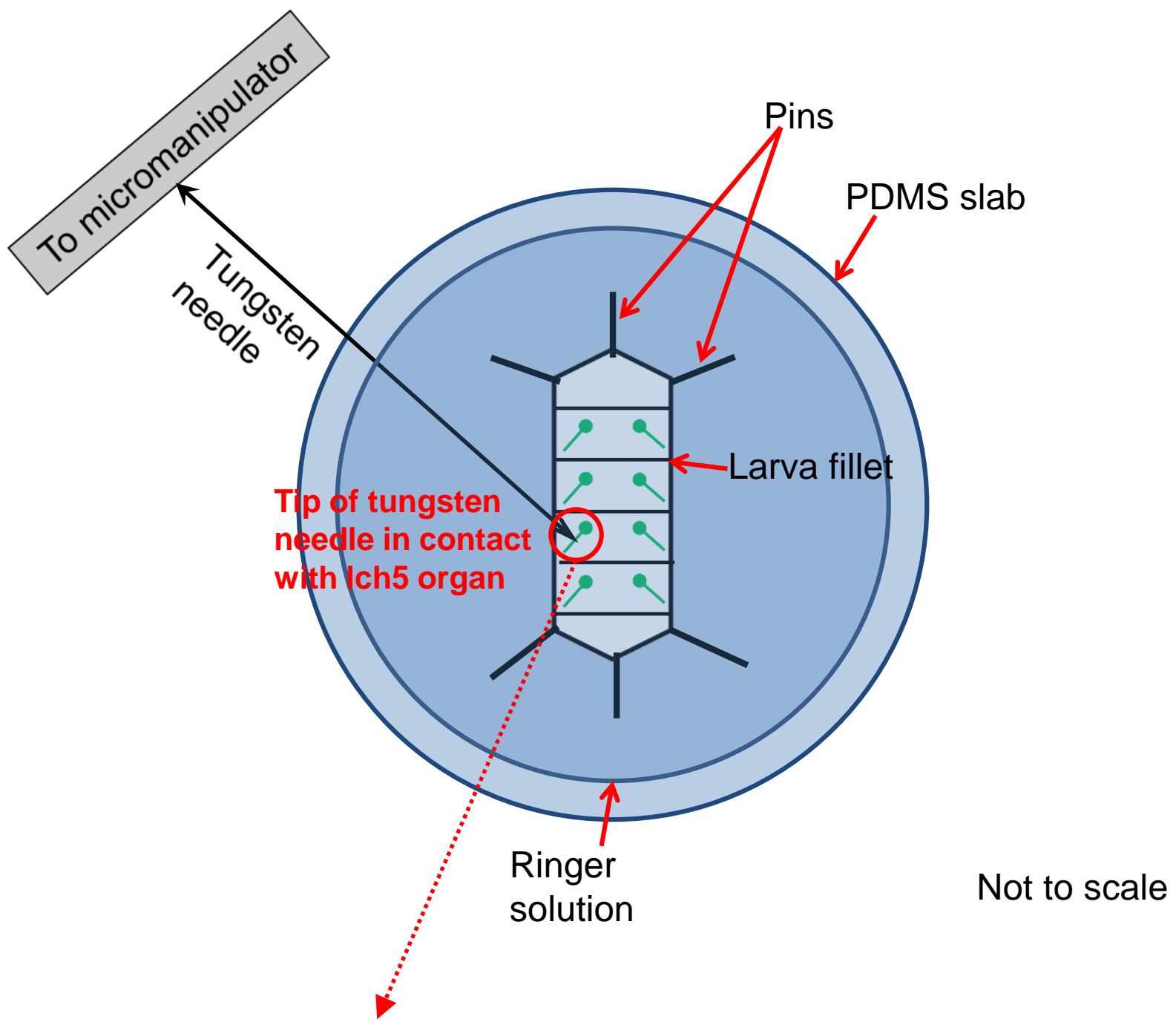

CA \& LA: end points of Ich5 organ
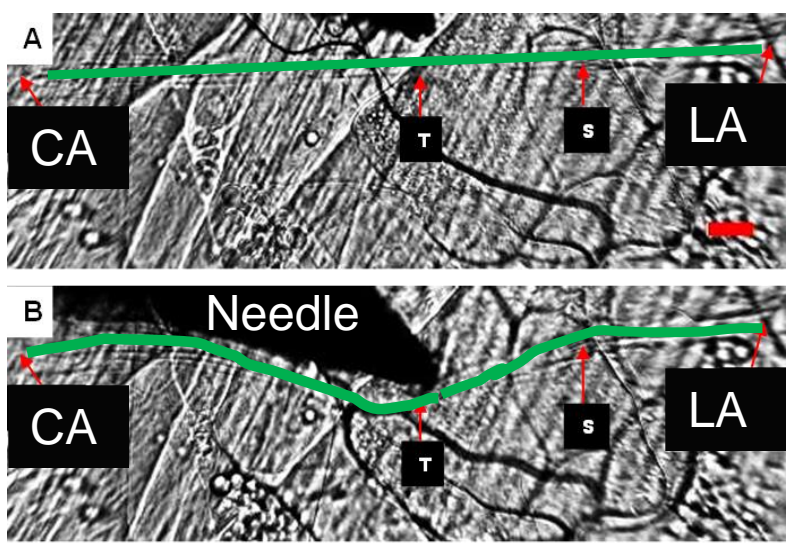

Fig. 3.8.2. Schematic depiction of the relaxation kinetics and shape experiments as described in sections 4.4 and 4.5 . The green curve in the inset depicts the change in shape of the Ich5 organ as it is displaced by the needle. For more details, refer Fig. 4.5.1 in Results.

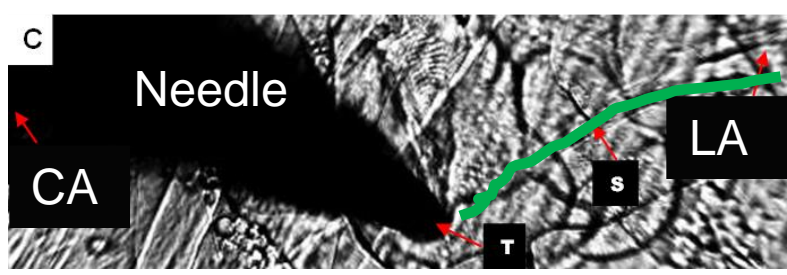




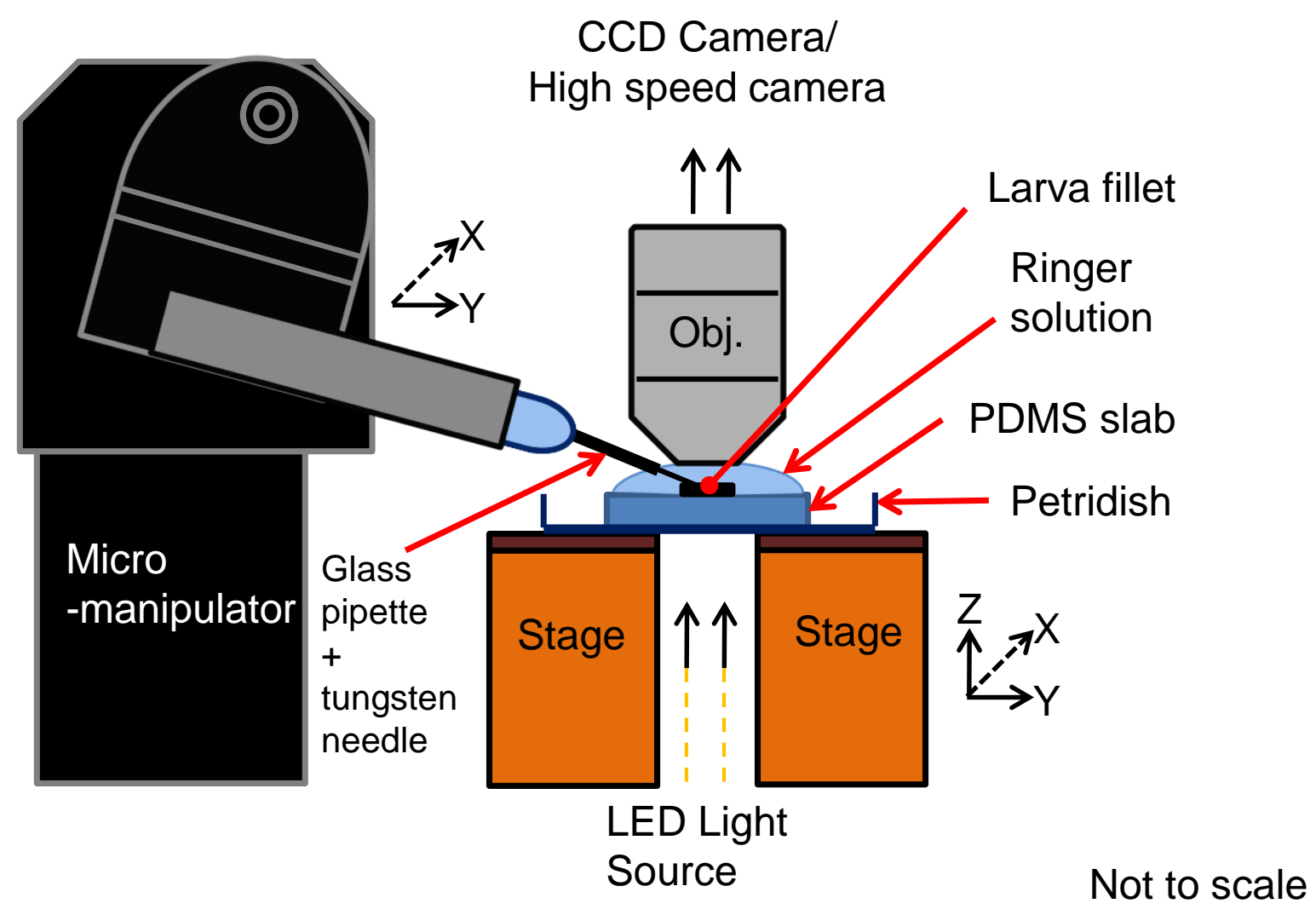

Fig. 3.8.3. Depiction of the set-up used for the relaxation and shape experiments described in sections 5.4 and 5.5, showing the relative placement of various components. Image modified from Dr Christopher Battle.

\subsection{Laser ablation}

Laser ablation can be defined as using a high-power laser to create an incision or a hole in a material, whether biological or not. The technique is practically as old as lasers themselves, with several discoveries in the 1960s paving the way for ablation techniques that are in use today (141). In the life sciences, laser ablation has a wide range of applications, which we shall discuss in some detail below, before moving on to an introduction to the laser ablation experiments that form a part of this thesis. Vogel and Venugopalan, in their 2003 review, define ablation in a biological context as "any process of tissue incision or removal, regardless of the photophysical or photochemical processes involved" (142).

The foundation for lasers in experimental science was laid by Maiman in 1960, and researchers were quite keen to delve into the possibilities of manipulating and destroying biological tissues using lasers $(142,143)$. Since then, several investigations were carried out especially in the fields of dermatology and ophthalmology $(142,144-147)$. However, the first experiment that was medically feasible was carried out in the 1970s $(142,148)$. In more recent times, several 
biophysical experiments have been conducted in connection to the actomyosin cortex. Examples of such investigations are discussed below.

Many discoveries in developmental biology have been made using laser ablation. Mechanical responses to laser ablation of various tissues in the Drosophila embryo have been studied, and the role of these tissues in dorsal closure has been investigated (149). In the nematode worm Caenorhabditis elegans, four specific actin-rich cells have been shown to be required for the process of ventral closure, by the fact that laser inactivation of these cells abolishes ventral closure (150). Laser nano-dissection has been used to examine the mechanics of Drosophila embryonic elongation (151). Laser ablation has also been used to create an opening in the zona pellucida of the mouse embryo without interfering with formation of the blastocyst (152). Wound healing in the Drosophila embryo has been shown to have striking parallels to the mechanism of dorsal closure (153).

Stress fiber mechanics have been extensively studied by ablation. Ablating a single stress fiber has been shown to be more detrimental to cell shape and cytoskeletal mechanics than ablating multiple fibers at once (154). Laser nanosurgery has been employed to map variance in stress fiber mechanics in different regions of the cell (155). The data from Kumar et al. (154) has been the basis of a predictive model of stress fiber mechanics (156).

Microtubule mechanics have also been investigated (157). Forces arising due to friction and dynein motors have been shown to have a greater effect on microtubule shape than elastic forces do (158). In the mitotic spindle, it has been shown that severing kinetochore fibers using a laser leads to them growing back towards the outside (159). Rates of growth, contraction and rescue of microtubules have been measured concurrently in living cells (160).

Cell deformation and migration is another area where laser ablation has found far-reaching application. Focal laser ablation has been used to generate blebs at different positions on a cell and find that bleb expansion is cortical tension-dependent and requires a minimum threshold tension to take place (161). MDCK cells have been shown to migrate actively under the guidance of a "leader" MDCK cell, and laser ablation has shown that the tensile stress is greatest at the position of this "leader" cell (162). A laser has been used to induce inflammation in the Drosophila embryo, and enzymes known as Rho-family small GTPases have been found to be critical to the motility of haemocytes migrating towards the site of inflammation (163). 
The laser ablation experiments in this thesis were done in collaboration with Deqing Kong in the group of Prof. Dr. Jörg Großhans at the Institute of Developmental Biochemistry, University Medical Centre, University of Göttingen, and also with Dr. Chonglin Guan (specifically the experiments related to Sqh-GFP and Sqh-RNAi X Pinta GAL4 larvae).

The larva was prepared by squeezing between a slide and a coverslip (as described in section 3.6 in Materials and Methods). A spinning disc confocal microscope (CSU-X1, Carl Zeiss) was used to visualize the Ich5 organ with a 100X oil objective (NA 1.4, Plan-APOCHROMAT, Carl Zeiss). Some experiments were performed with a 40X oil objective (NA 1.3, EC Plan-NEOFLUAR, Carl Zeiss), to obtain a large enough field of view to image the complete retraction of the cap cells (see Results and Discussion). The slide was placed such that the coverslip was in contact with the oil on the objective (Fig. 4.6.2). Ablation was carried out using a $355 \mathrm{~nm}$ pulsed YAG UV laser, average power $14 \mathrm{~mW}$ (Rapp OptoElectronic, Wedel, Germany) in click-and-fire mode, with 20\% intensity and $300 \mathrm{~ms}$ pulse duration per click. The energy delivered was $\sim 0.84 \mathrm{~mJ} /$ pulse. The experiments were controlled by the ROE SysCon-Zen software (Rapp OptoElectronic).

The laser was focused either just in front of the scolopales, at the initial part of the cap cells, on the neuronal dendrites between the scolopales and the neuronal somata, or on the ligament cells. The laser was aimed either at one scolopidium at a time (point focus) or at all scolopidia together (line focus). Videos of the recoil of the organ following ablation were recorded with an AxioCam MRm camera (Carl Zeiss) at a rate of $1 \mathrm{fps}$.

We used fluorescence microscopy, so that only the neurons and the scolopales were visible in the larvae (in case of Sqh-GFP larvae, it was the cap cells and ligament cells that were visible). However, In case of the myosin mutants and controls, we used brightfield illumination, since the lch5 organs were not labeled. While this did not give us as clear a view as in case of the fluorescence imaging, we could still see the important structures, and easily compare the videos to the ones recorded using fluorescence. The set-up is shown in Fig. 3.9.2. 


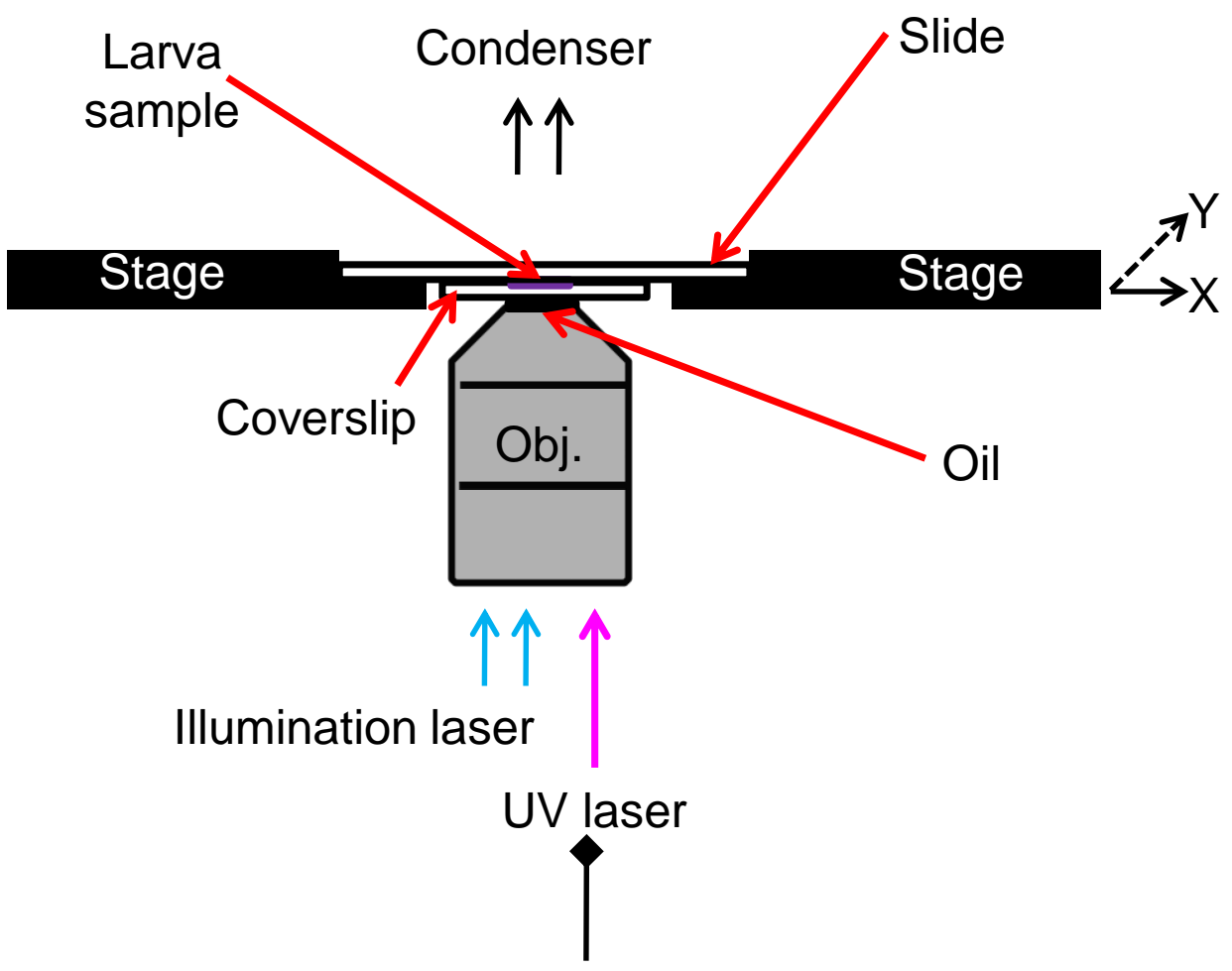

ROE SysCon-Zen

software

Fig. 3.9.2. Set-up used for the UV laser cutting experiments, showing the relative placement of components. 


\subsection{Force measurements using calibrated needles}

This is the last set of experiments that was performed, in collaboration with Christian Kreis in the group of Prof. Dr. Oliver Bäumchen at MPI-ds, Göttingen, and Dr. Chonglin Guan. For this, a large glass slide was taken, and a PDMS layer was coated on it (see section 3.8.2 for details on PDMS preparation). A larva fillet with excised muscles (section 3.8.4) was prepared and oriented as shown in Fig. 3.10.2 A. Two cut O-rings were placed around the fillet. The space was filled with Ringer solution, and another glass slide of similar size as the first one was clamped on top (Fig. 3.10.1). Into the fluid cell, a specially-made L-shaped glass pipette, prepared by C. Kreis, was inserted using a micromanipulator. The pipette had a long arm, a short arm, and a microscopic tip perpendicular to both arms. Figs. 3.10.1 and 3.10.2 illustrate the shape of this pipette. The tip of the pipette was brought in contact with the Ich5 organ as shown in Fig. 3.10.2 B. Then, the sample stage was moved in a direction perpendicular to the Ich5 organ, such that the Ich5 organ was pushed against the pipette tip. At some point, the pipette tip automatically disengaged from the lch5 organ, and the organ relaxed back. In an ideal situation, the pipette tip would get deflected and from this deflection one can measure the force exerted by the organ on the pipette. Some preliminary results were obtained (section 4.7).

Videos were recorded using a custom-made set-up at the lab of Prof. Bäumchen. Some videos were additionally recorded at a set-up built by Dr. Guan at the Third Institute of Physics. Here, we used the inbuilt software provided by Scientifica to operate the micromanipulator. Rather than move the stage, we moved the glass pipette and deflected the lch5 organ by known distances.

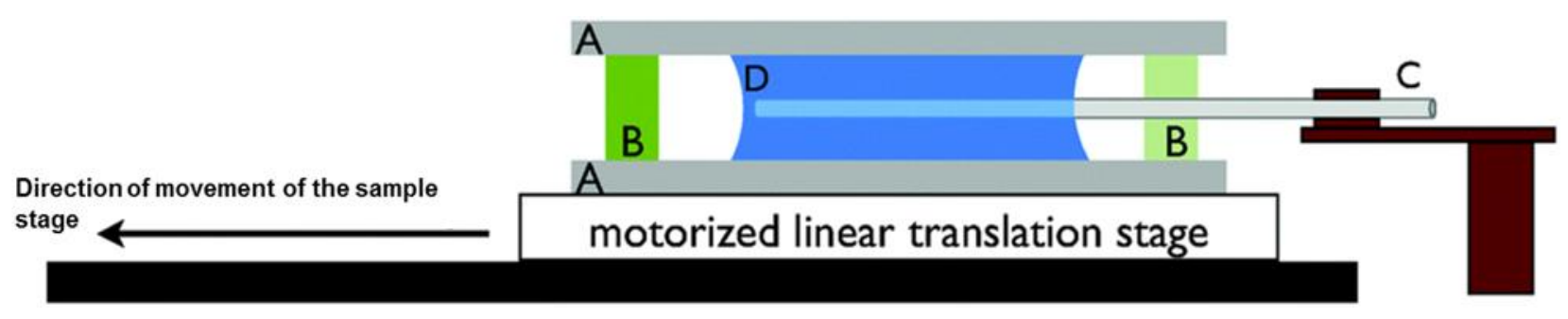

Fig. 3.10.1. Set-up used for the force measurement experiments. CD is the long arm of the pipette. The short arm is perpendicular to $C D$ at $D$, and goes into the plane of the paper. The tip is bent downwards, perpendicular to both the long arm and the short arm. A: glass slides, B: rubber O-ring. The larva sample is on the lower glass slide. Figure modified from Haefner, Bäumchen et al., 2015 (164). 

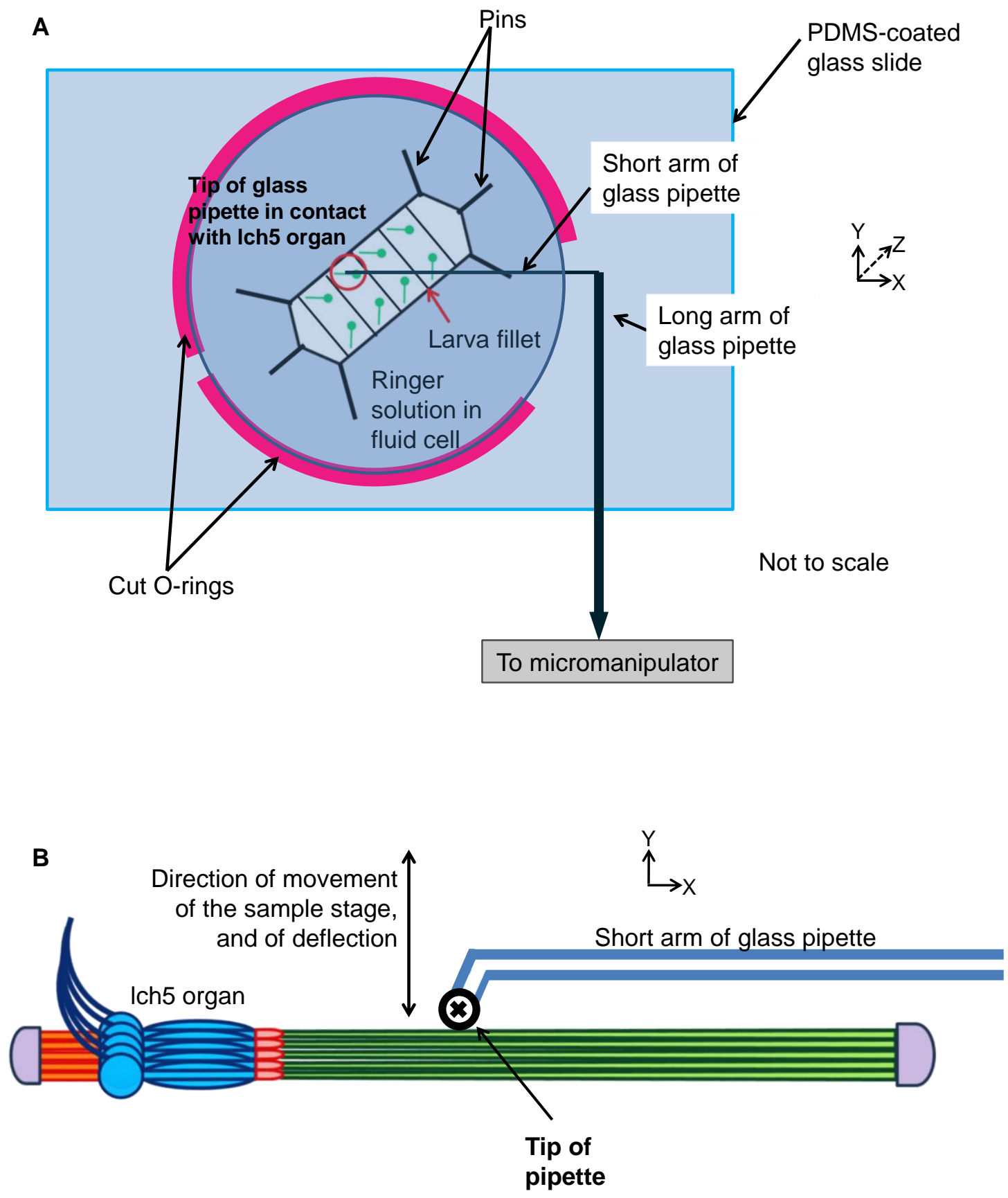

Fig. 3.10.2. (A) Orientation of the larva sample relative to the glass pipette (view from above). In this diagram, the larva has been enlarged several times from its actual size relative to that of the other components, in order to make its orientation clear. The bent tip of the pipette is not shown, since it goes into the plane of the paper. (B) A closer look at the glass pipette in contact with the lch5 organ (view from below). In practice, only the tip of the pipette is visible in the videos. The short arm is well above the focal plane. 


\section{Results and discussion}

\subsection{Measuring forces using optical tweezers}

We attempted to use optical trapping to (a) measure active fluctuations in the cilia of Johnston's or Ich5 organ using a trapped bead, and (b) deliver forces to these systems and measure the response by calcium imaging or electrophysiology. For these experiments, I used elaV-GCaMP6 flies and larvae. GCaMP6 is a calmodulin-tagged version of GFP, which increases its fluorescence upon encountering a calcium signal (165), and is thus specially suited for calcium imaging. The experiments did not yield the desired outcome, the reasons for which are discussed below.

One initial direction we explored was to measure fluctuations from Johnston's organ (JO) in the adult fly (Section 2.3). The origin of this idea lay in literature describing active amplification in the Drosophila ear that produces very similar results to what is observed in the vertebrate ear $(5,19$, $20,103)$. Several molecular motors are involved in this process, and we believed that the active fluctuations assumed to be generated by these motors in the chordotonal cilia of JO could be measured using optical trapping or a similar technique. Before taking a closer look into the mechanics of the $\mathrm{JO}$, we thought of studying the arista, since that is the structure that first receives the auditory stimulus before passing it on to the JO. We had hoped that an optically trapped bead would suffice for this purpose. I attempted the following experiment (Section 4.3.1): A fly head, or in some cases an intact fly, was stuck to the bottom of a petridish using a small amount of superglue, and the petridish was filled with water. We then added polystyrene beads using a pipette. Beads of $4 \mu \mathrm{m}$ and $8 \mu \mathrm{m}$ diameter were used. The beads were trapped using the IR laser, and the arista, visible as a branched structure, was moved closer to the trapped bead. However, as soon as the arista came in contact with the laser, there was a large bubble, owing to thermal damage (Fig. 4.1.1). Also, the arista is large in size and rigid, and we could not use an optically trapped bead to displace it. Another issue was that when placed under water, the antenna was not in an alive state anymore, and so the experiment did not yield any useful information. We also repeated the experiment with the somatosensory bristles on the mouth parts (Fig. 4.1.2). 


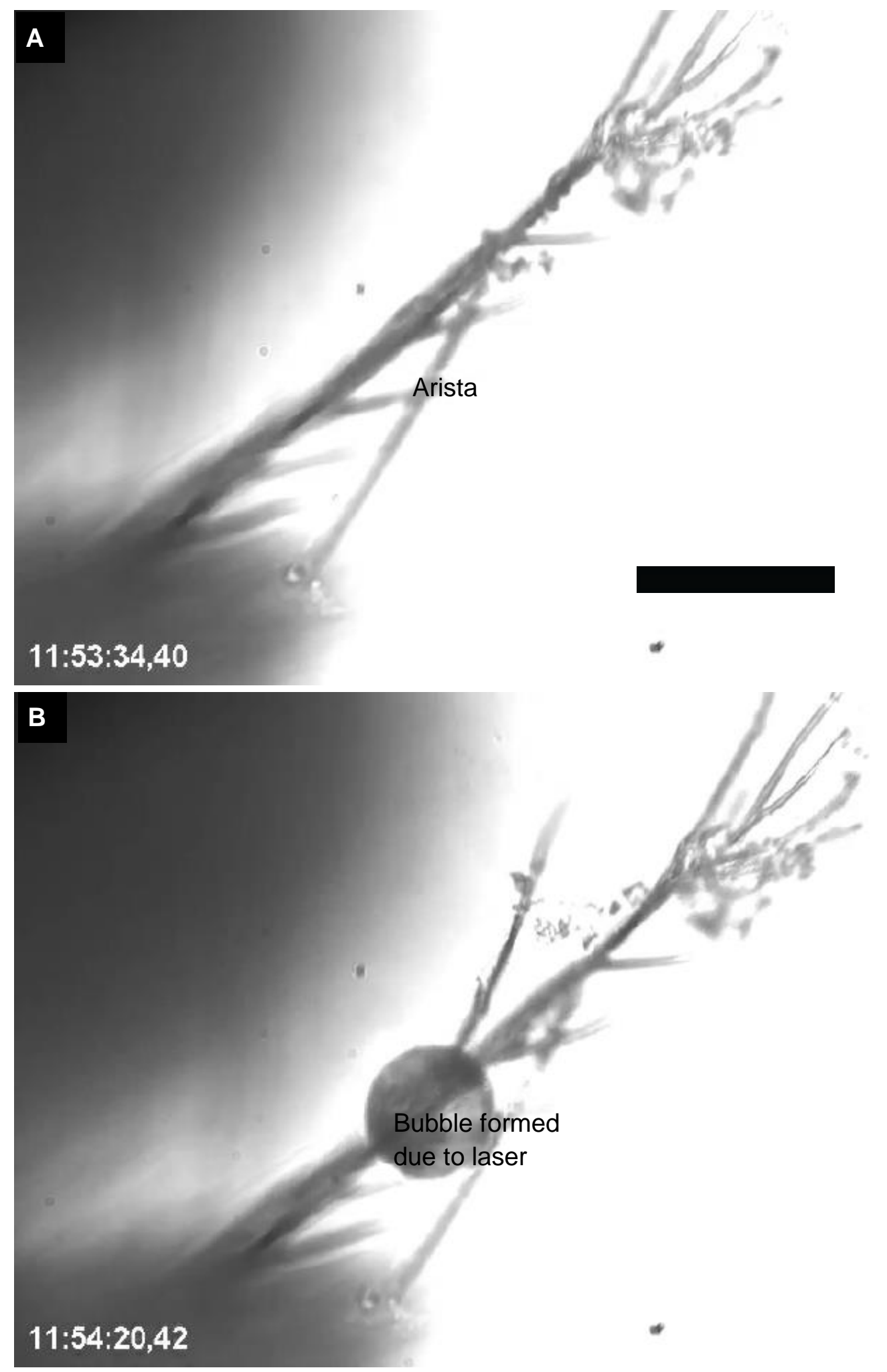

Fig 4.1.1. The arista before and after bringing in contact with the optically-trapped bead. (A) Before trapping (B) A bubble formed on the arista at the point where it was in contact with the bead, indicating thermal damage. Scale bar $=80 \mu \mathrm{m}$. 


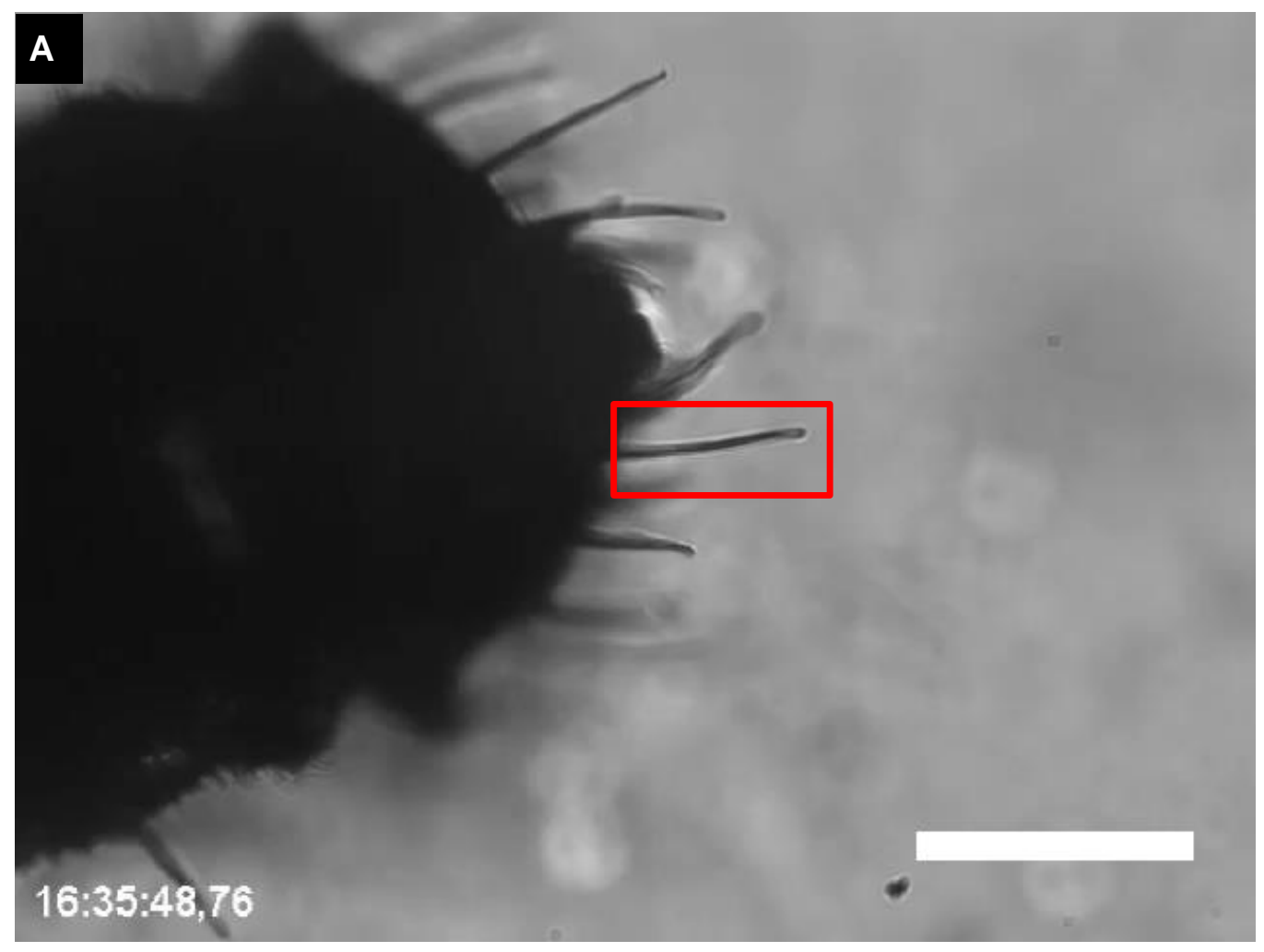

\section{B}

$16: 36: 09,54$

Fig 4.1.2. A bristle on the mouthparts indicated by the red rectangle (A) Before trapping (B) The bristle in a bent configuration, indicating thermal damage. Scale bar $=80 \mu \mathrm{m}$. 
As for probing the mechanics of the $\mathrm{JO}$ neurons directly, we soon understood that this would not be possible using optical tweezers. In order to come in direct contact with the neurons, we would have to puncture the pedicel of the antenna, in which the $\mathrm{JO}$ is housed. This would alter the mechanics of the organ. We then decided to change our approach. After detailed discussions with our collaborators (the group of Prof. Dr. Martin Göpfert at the Schwann-Schleiden Center, Faculty of Biology, University of Göttingen), and a study of literature, mainly Zhang et al. 2013 (44), we changed gears and shifted our interest to the lch5 organ, which one can access much more easily in a filleted larva (Section 4.4.4). Since this is a chordotonal organ as well, albeit less complex than the $\mathrm{JO}$, we assume that understanding its mechanics will give us a better understanding of the functioning of chordotonal organs in general. Given that active amplification is thought to be the basis of functioning of the $\mathrm{JO}$, we speculated whether such a process exists in the Ich5 organ and whether the fluctuations arising from molecular motors can be measured directly in this organ. Since the cilia were located inside the scolopales, we could not get direct access to them. Hence we needed a method to measure the fluctuations externally.

We proceeded to try to measure these fluctuations using optical tweezers, with the aim of placing a bead near the scolopales and trapping it. In our initial trials we were not successful, because the muscles acted as a barrier between the lch5 organ and the bead, and once the bead came in contact with the muscles it could not be trapped anymore. The power spectra (refer section on optical trapping) of the bead floating in the Ringer buffer and closer to the scolopales of the lch5 organ were not highly different from one another (Fig. 4.1.3). If there were active mechanical fluctuations emanating from the chordotonal cilia, the power spectrum closer to the scolopales would have appeared different from the case where the bead was away from the scolopales, because the fluctuations from the cilia would have added to the passive Brownian fluctuations (137). However, this was not observed. 


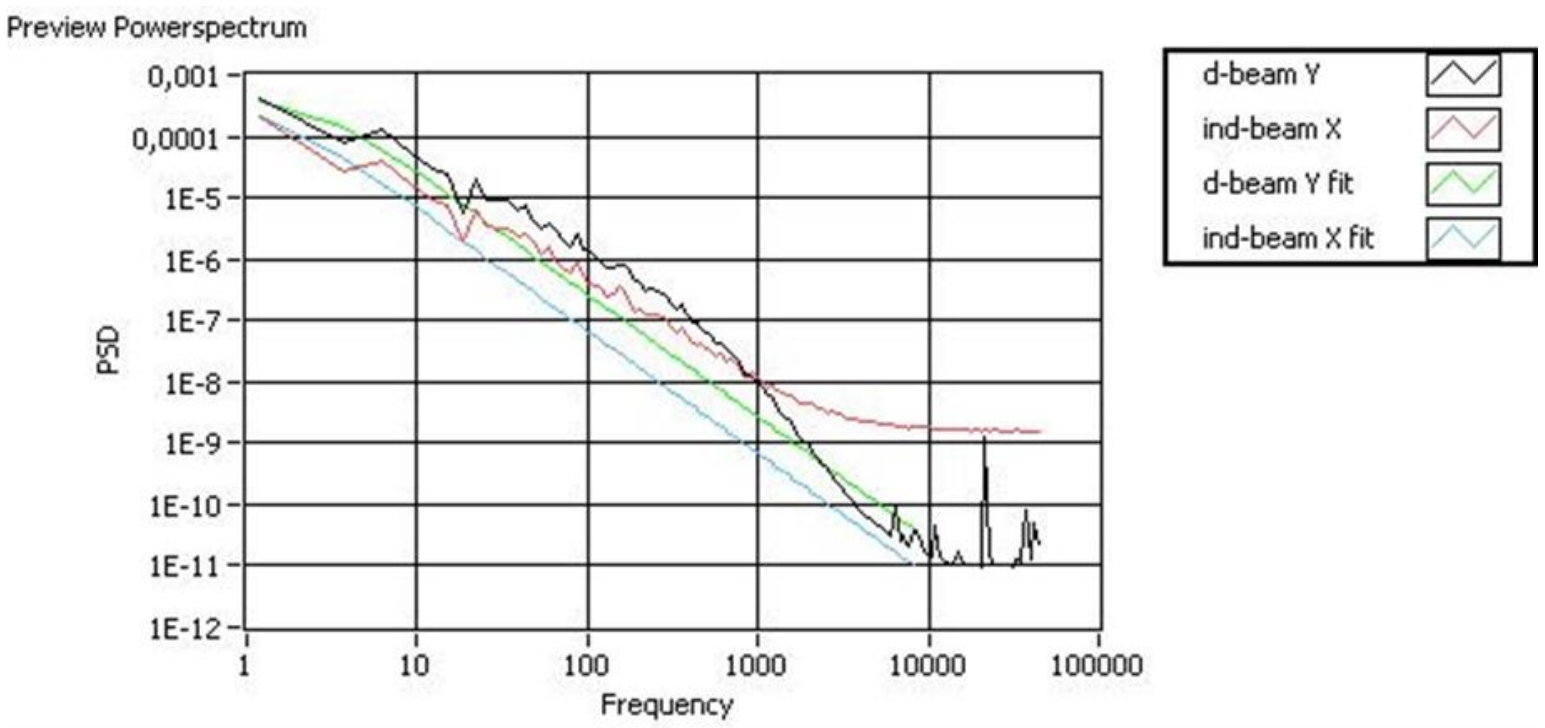

\section{Bead in Ringer}

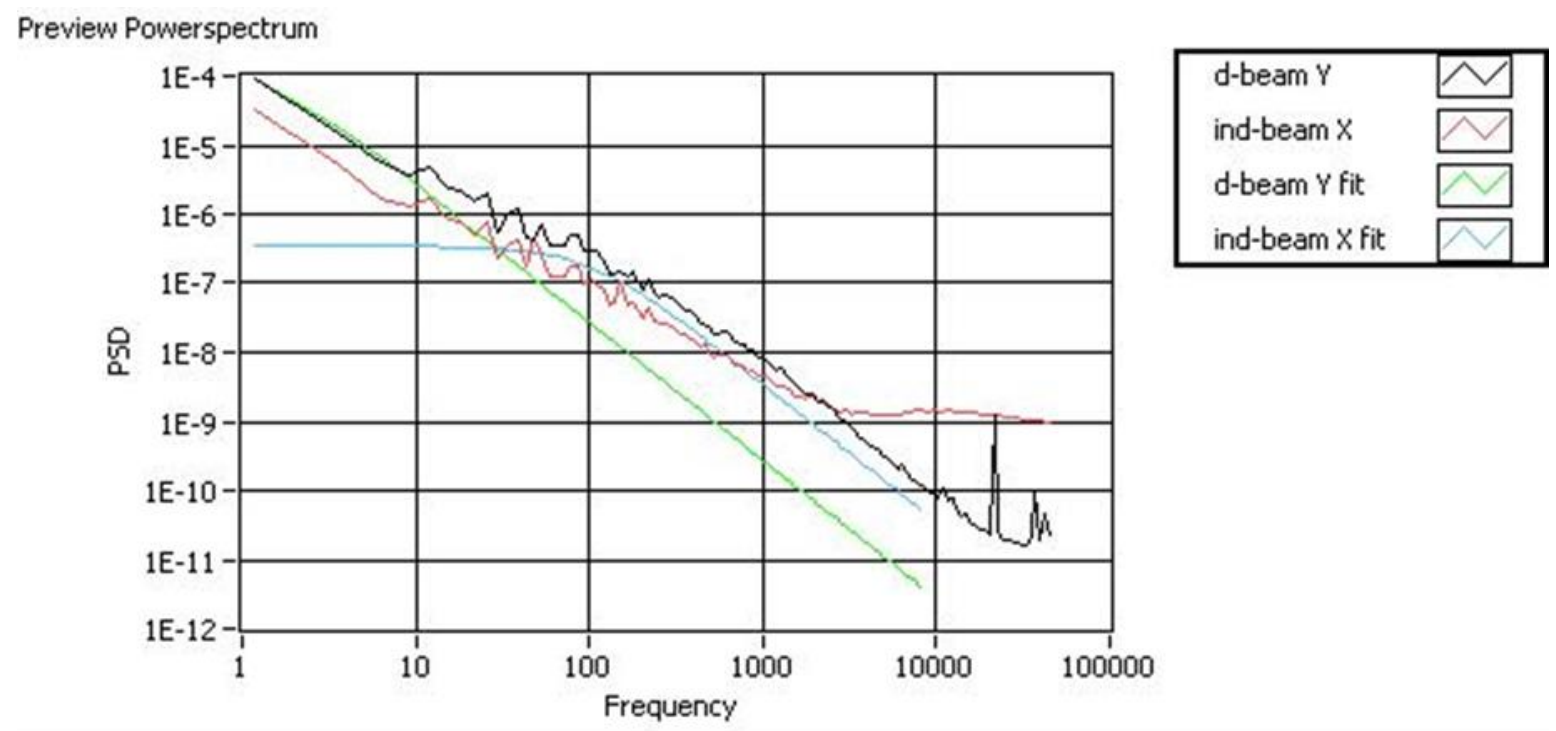

Bead near muscles

Fig 4.1.3. Power spectral densities of optically-trapped beads (A) Bead floating in the Ringer buffer (B) Bead trapped nearer to the Ich5 organ 
Our next attempt was to reach the Ich5 organ by pushing a glass pipette under the muscles and injecting beads onto the organ. This was done by "weaving" the pipette through the muscles (as per Dr. Ben Warren at the group of Prof. Dr. Göpfert) (Figs. 4.1.4 and 4.1.5), but here too we failed to trap them with the laser.

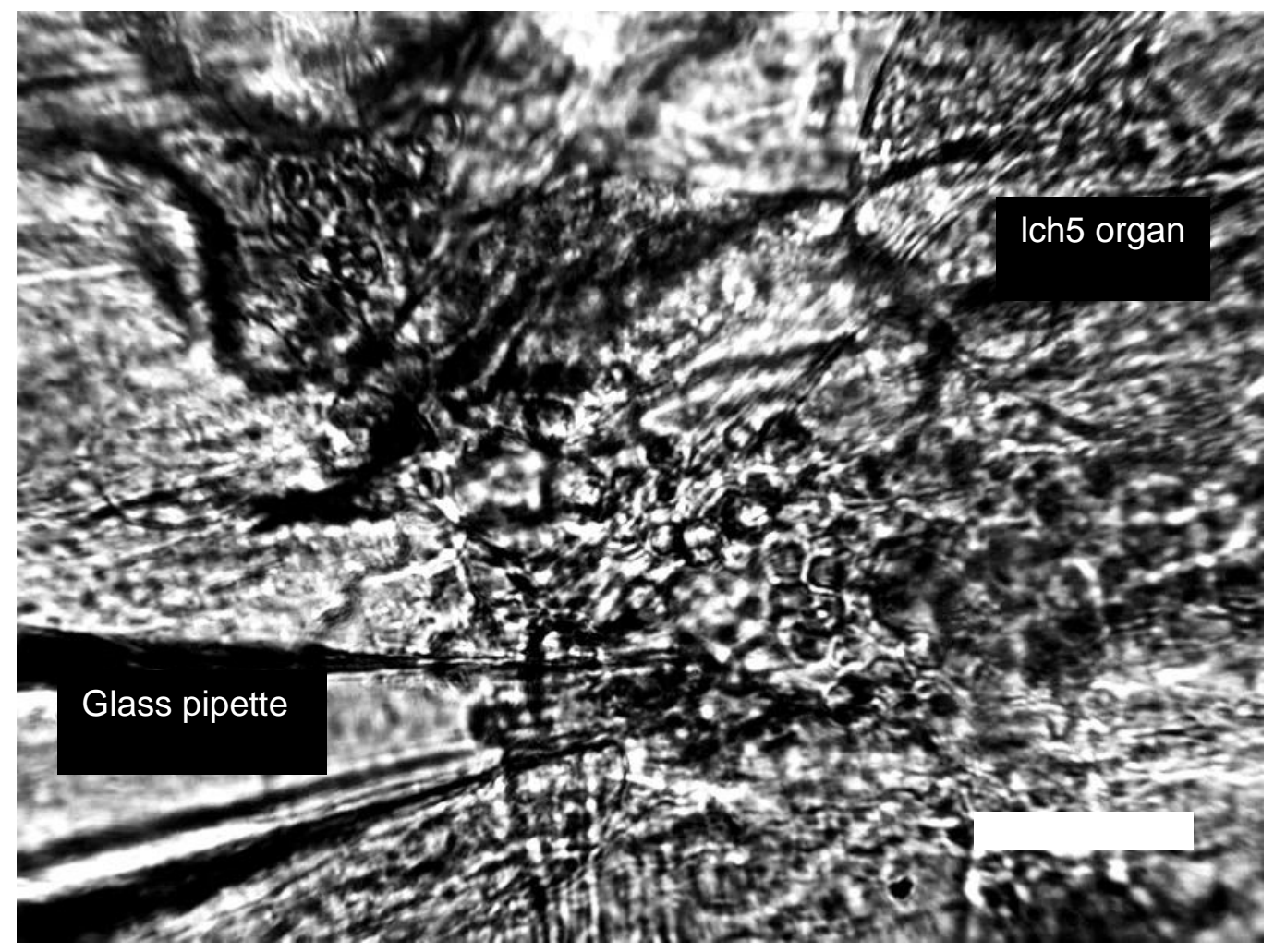

Fig 4.1.4. The glass pipette in contact with the Ich5 organ. Polystyrene beads are also visible. Scale bar $=50$ $\mu \mathrm{m}$. 


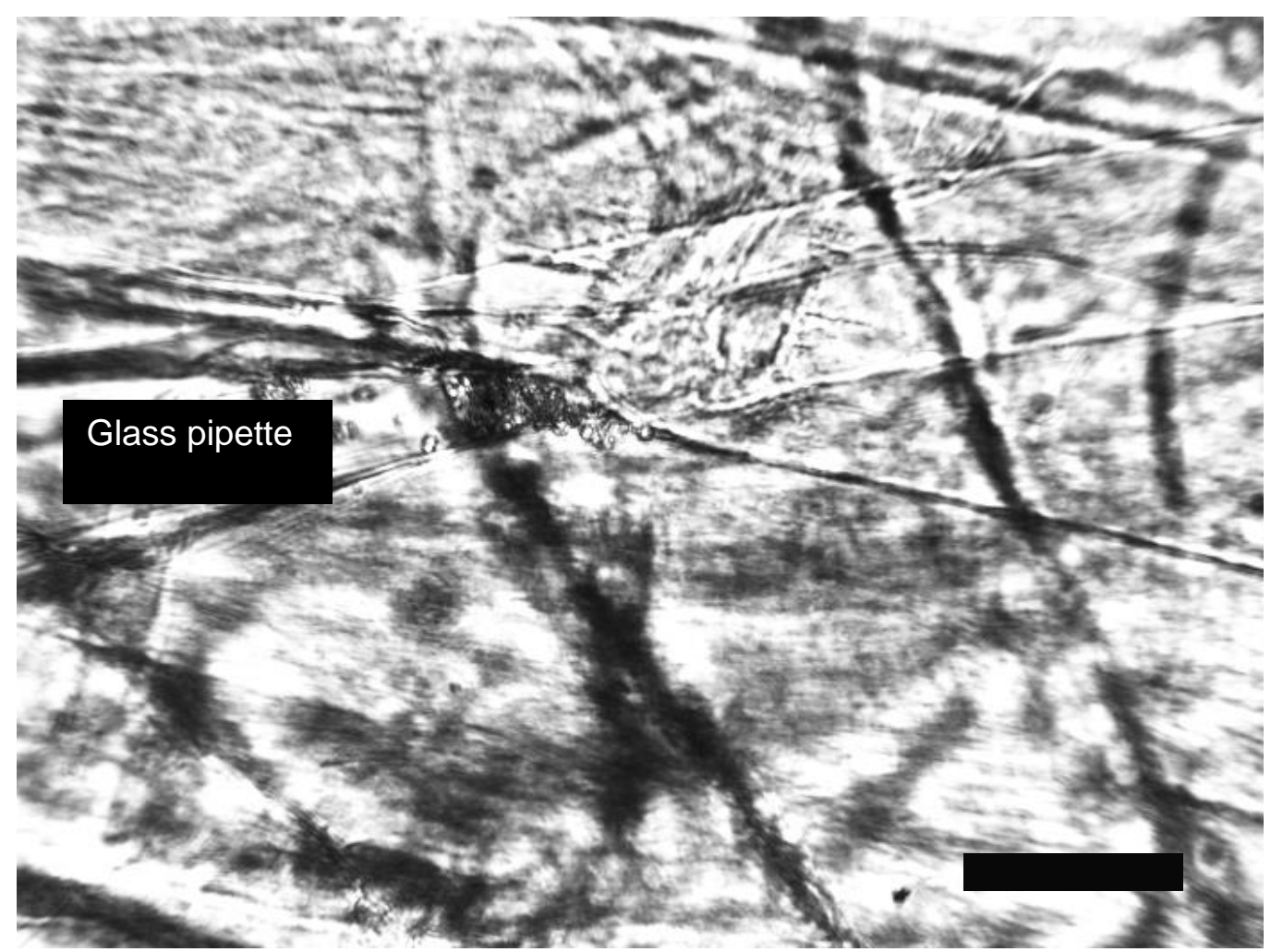

Fig. 4.1.5. Weaving the pipette through the muscles. Scale bar $=50 \mu \mathrm{m}$. 

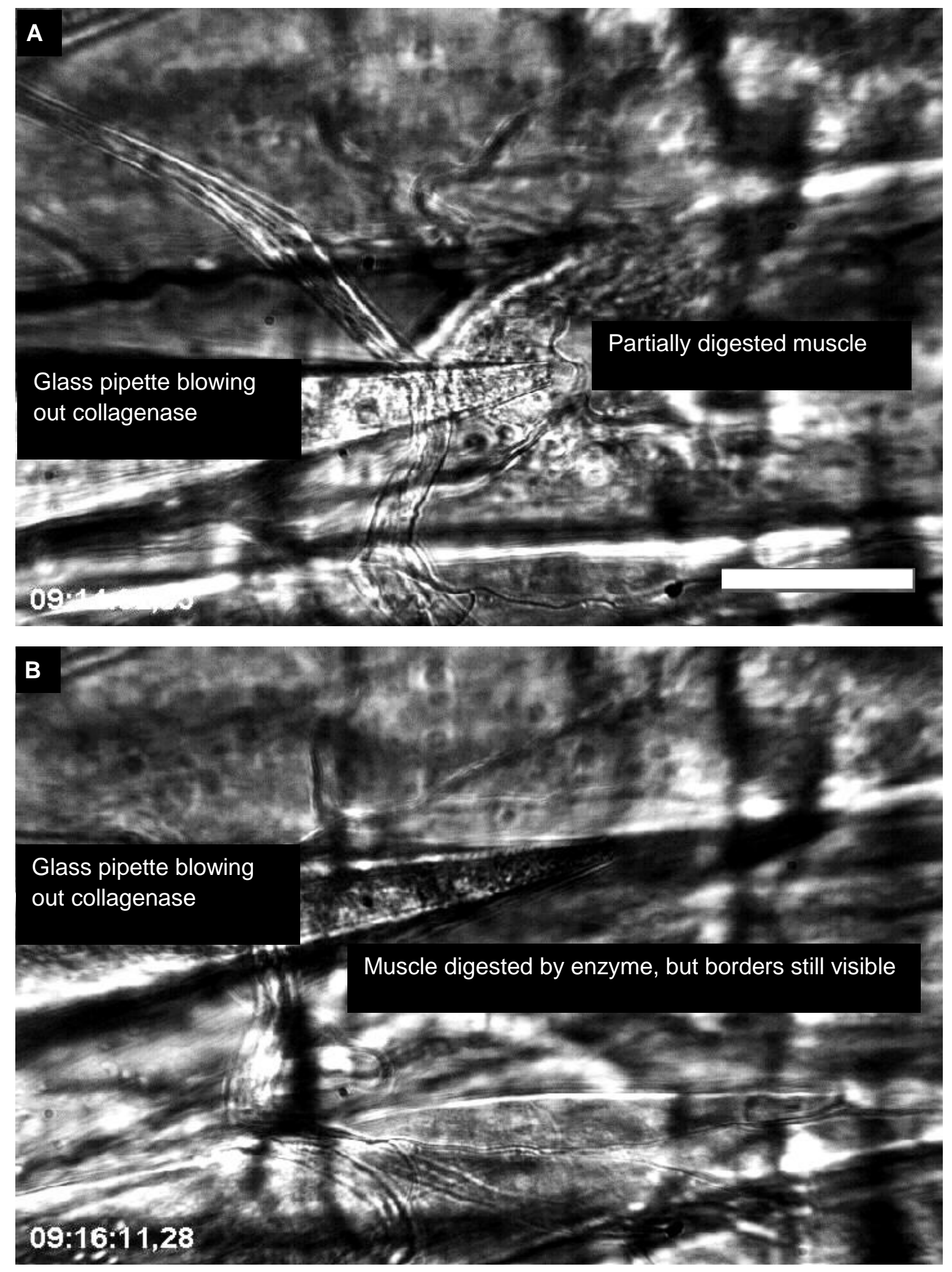

Fig. 4.1.6. (A) A longitudinal muscle partially digested by collagenase. (B) Muscle fully digested, but a transparent band of tissue was still left behind. Scale bar $=50 \mu \mathrm{m}$.

In one of our trials we were indeed successful in freeing the organ, after collagenase treatment and pulling and pushing of the muscles with the glass pipette, and we also managed to get a bead in contact with the organ, but this bead could not be trapped anymore once it was stuck to the organ (Fig. 4.1.7), and thus no fluctuations could be measured. Also, we did not have direct access to the 
cilia - since they are enclosed in the scolopales. Furthermore, the chordotonal organs are much larger than typical sizes of beads used in trapping experiments. We then decided to modify our approach and study the mechanics of the entire lch5 organ, using laser ablation and mechanical manipulation with tungsten needles.

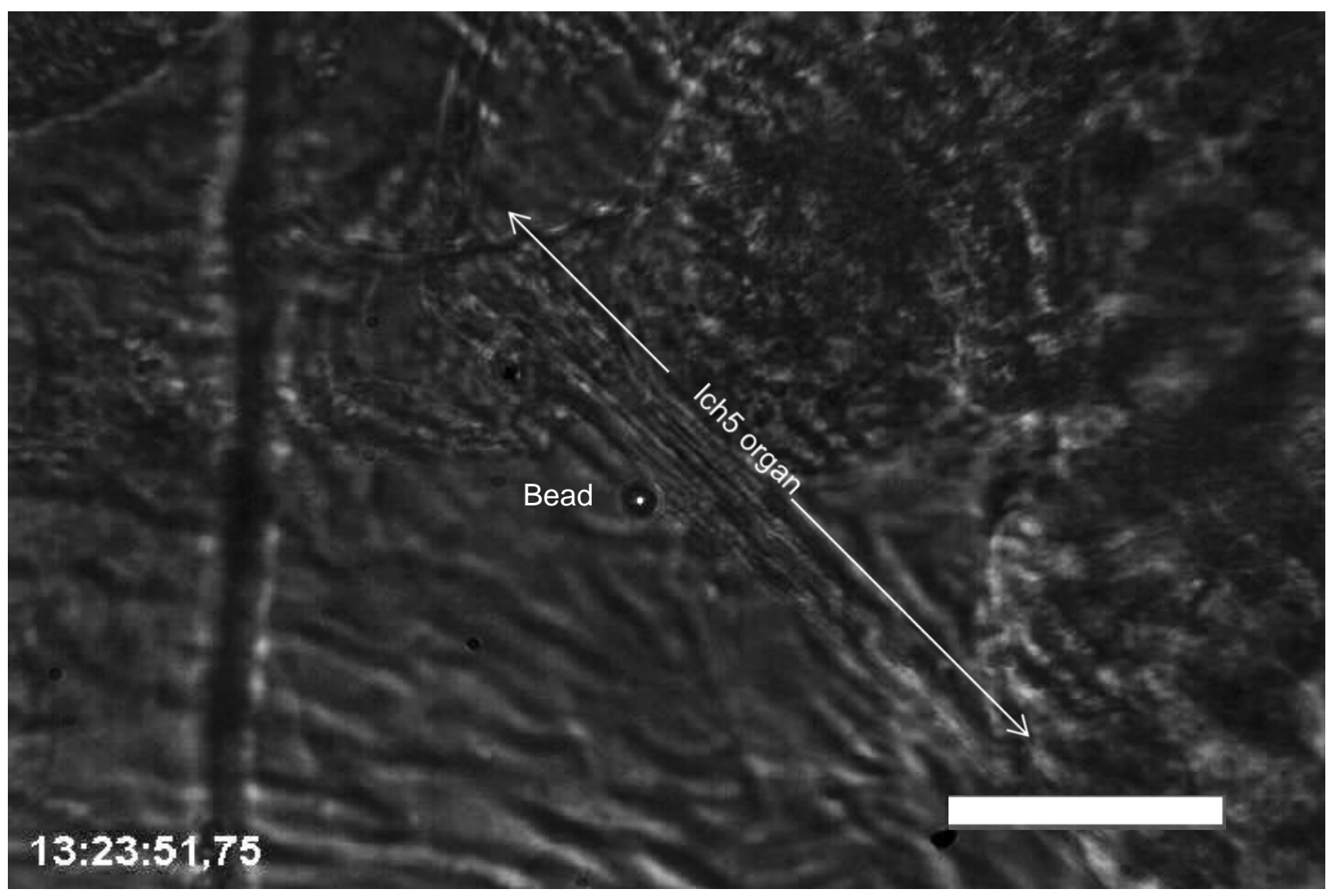

Fig. 4.1.7. A $4 \mu \mathrm{m}$ polystyrene bead in contact with the dendrites of the lch 5 organ. We observe that it is stuck to the organ and cannot be trapped anymore. Scale bar $=50 \mu \mathrm{m}$. 


\subsection{Study of crawling motion in the larva}

We placed larvae in a groove in an agarose gel coated on a glass slide (Fig. 4.3.1 in Materials and Methods), and observed the deformations of the cuticle in the segments, on the ventral side. At every segment boundary, there are rows of spike-like structures called cuticular denticles (166)

(Fig. 4.2.1 A). These denticles presumably provide the larva with a grip on the substrate as it crawls. The denticle rows were seen under the microscope (details) to undergo torsion and move closer together to form bunches. These denticle bunches were instrumental in pulling the segment boundaries closer together by undergoing torsion and exerting a push. The segment size was seen to reduce by $\sim 50 \%$ (Fig. 4.2.1 B\&C, Fig. 4.2.2 B). Also, a groove was seen to form on approximately the midline of the segment, and to deepen as the segment contracted (Fig. 4.2.1 $\mathrm{A} \& \mathrm{~B})$. To assess how these segment contractions influence the lch5 organ would require a mechanical model for their coupling. I initially used objective oil (section 3.1) as a substrate with reduced friction for the larva to crawl, and obtained some videos this way, but since it is better to have a situation closer to what the larva would experience in nature, I switched to using agarose gel (section 3.5). The videos obtained this way were of sufficient quality. 

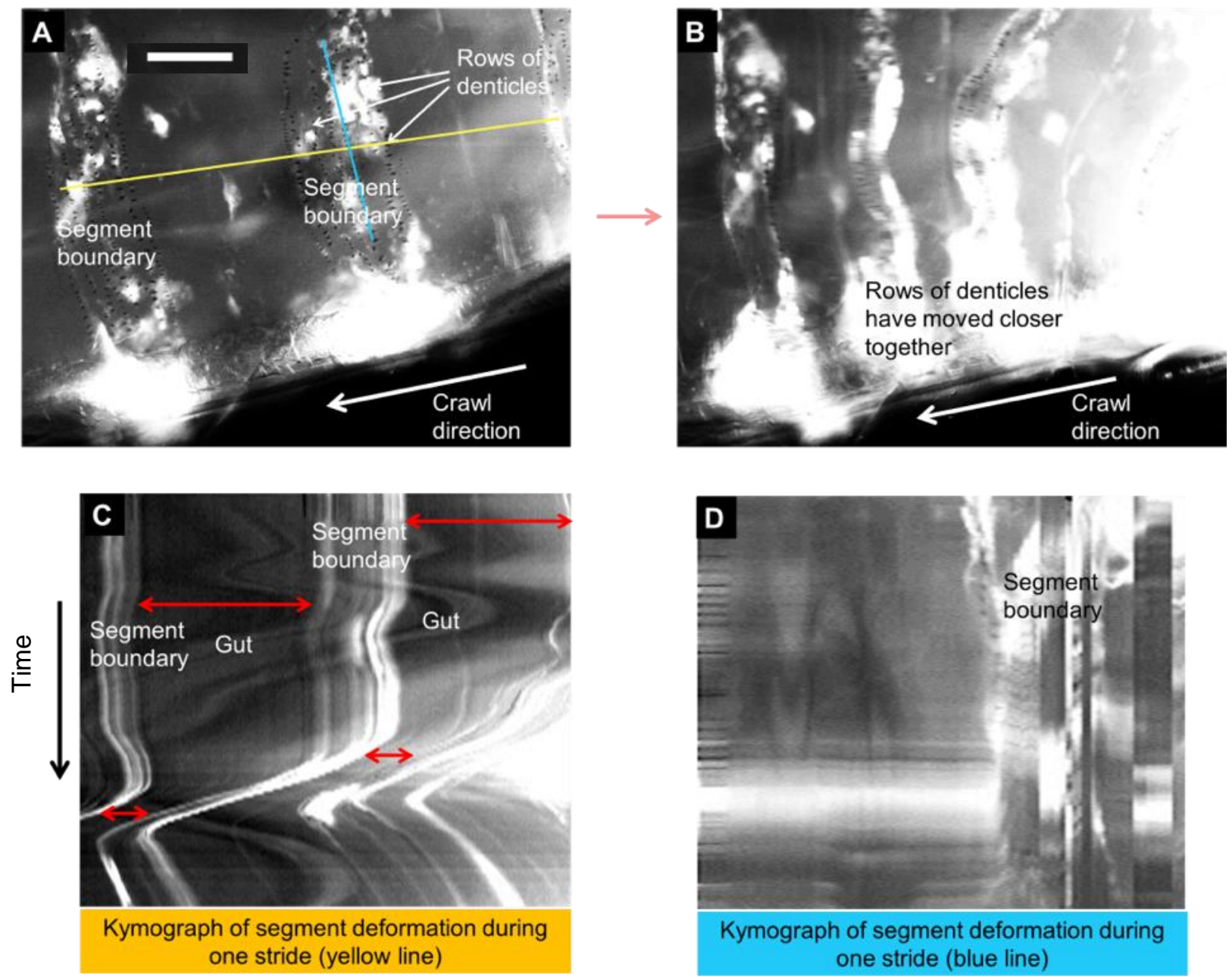

Fig. 4.2.1. Segment deformation in the Drosophila larva during crawling. The ventral side of a third instar larva is pictured, viewed under 20X magnification. It is crawling on objective oil. Scale bar $=0.2 \mathrm{~mm}$. (A) Prior to segment contraction. Cuticular denticles and segments are visible. (B) Contraction of the segments. The denticle rows have bunched up and there is torsion in the bunches. The segment length has reduced. (C) Kymograph indicating the contraction of the segment with time, plotted along the larva's body axis (yellow line in (A)). The double-headed red arrows denote the segment length. (D) Kymograph of the segment deformation, plotted along the axis of the segment boundary (blue line in $(A)$ ). 


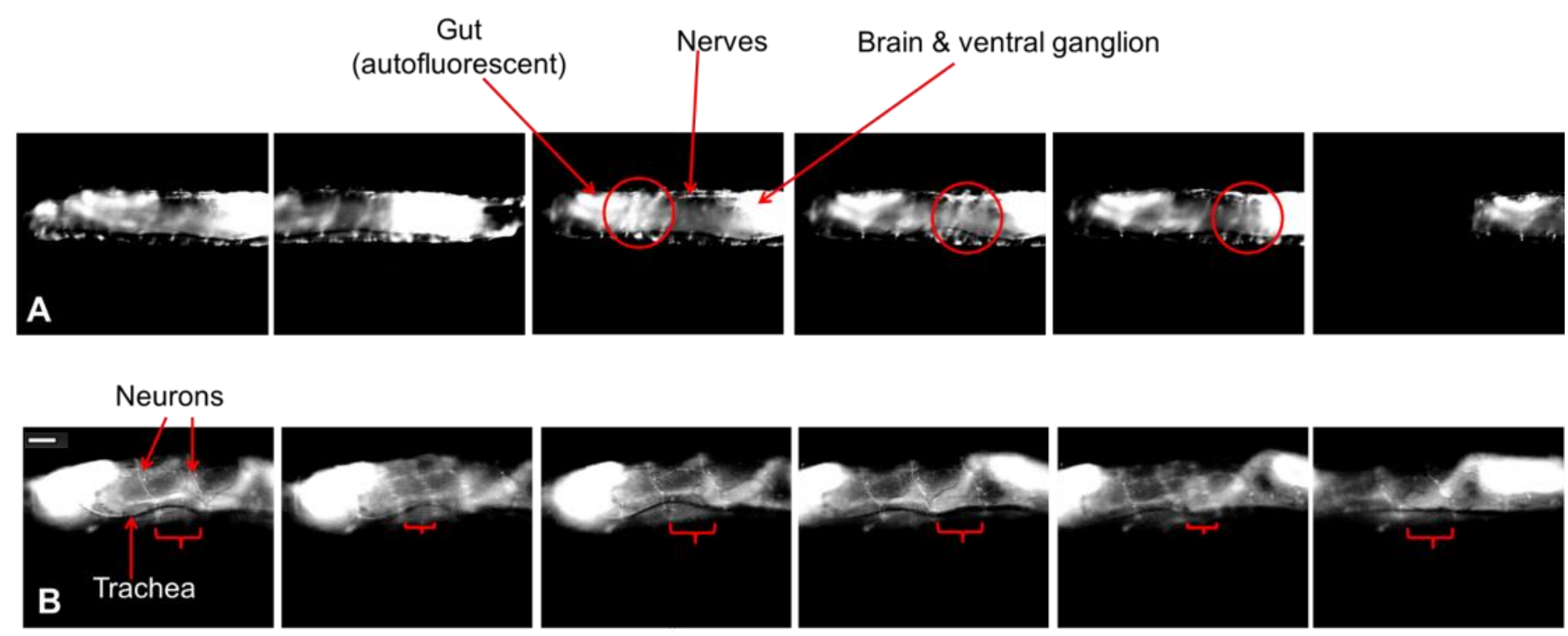

Fig. 4.2.2. Segment deformation in the Drosophila larva during crawling (lateral view). (A) The red circle indicates the position of the contracting segments, and shows the direction of the propagating peristaltic wave. (B) The red flower bracket depicts the segment boundaries moving closer together as the segment contracts. Scale bar $=0.5 \mathrm{~mm}$.

\subsection{DIC images of the Ich5 organ}

We next wished to obtain high-magnification and high-contrast images of the cuticle, and different components of the Ich5 organ (section 3.7 in Materials and Methods. Differential Interference Contrast (DIC) microscopy was the best imaging method for this purpose. The fillet preparation was not suitable for these experiments, since pins were used for it, and detaching it from the PDMS dish would damage it. I therefore devised an alternative preparation method, the "squished prep" described in section 3.6. This way a thin enough sample was obtained for the purpose of carrying out DIC microscopy.

In these images, the attachment cells for the ligament and cap cells were visible as brush-like structures. High-contrast images of the ligament, neurons, cap cells and scolopales were obtained (Figs. 4.3.1-4.3.8). The scolopales were observed to be marked with dot-like structures (Fig. 4.3.3) at the end in contact with the cap cells (49). These dot-like structures were presumably the ciliary dilations (section 2.2). 
In one video which I recorded using this technique, I observed the muscles contracting and relaxing. The longitudinal muscles did not affect the lch5 organ in any way, but when the lateral transverse muscles contracted, the middle muscle was seen to exert a push on the scolopales of the organ. This appeared to indicate that the LT muscles were somehow directly influencing the lch5 organ. I did not observe this in subsequent experiments, however. Recent literature (167) suggests that it is only the cuticle that exerts force on the lch5 organ as it contracts, hinting at an indirect, more subtle influence of the muscles.

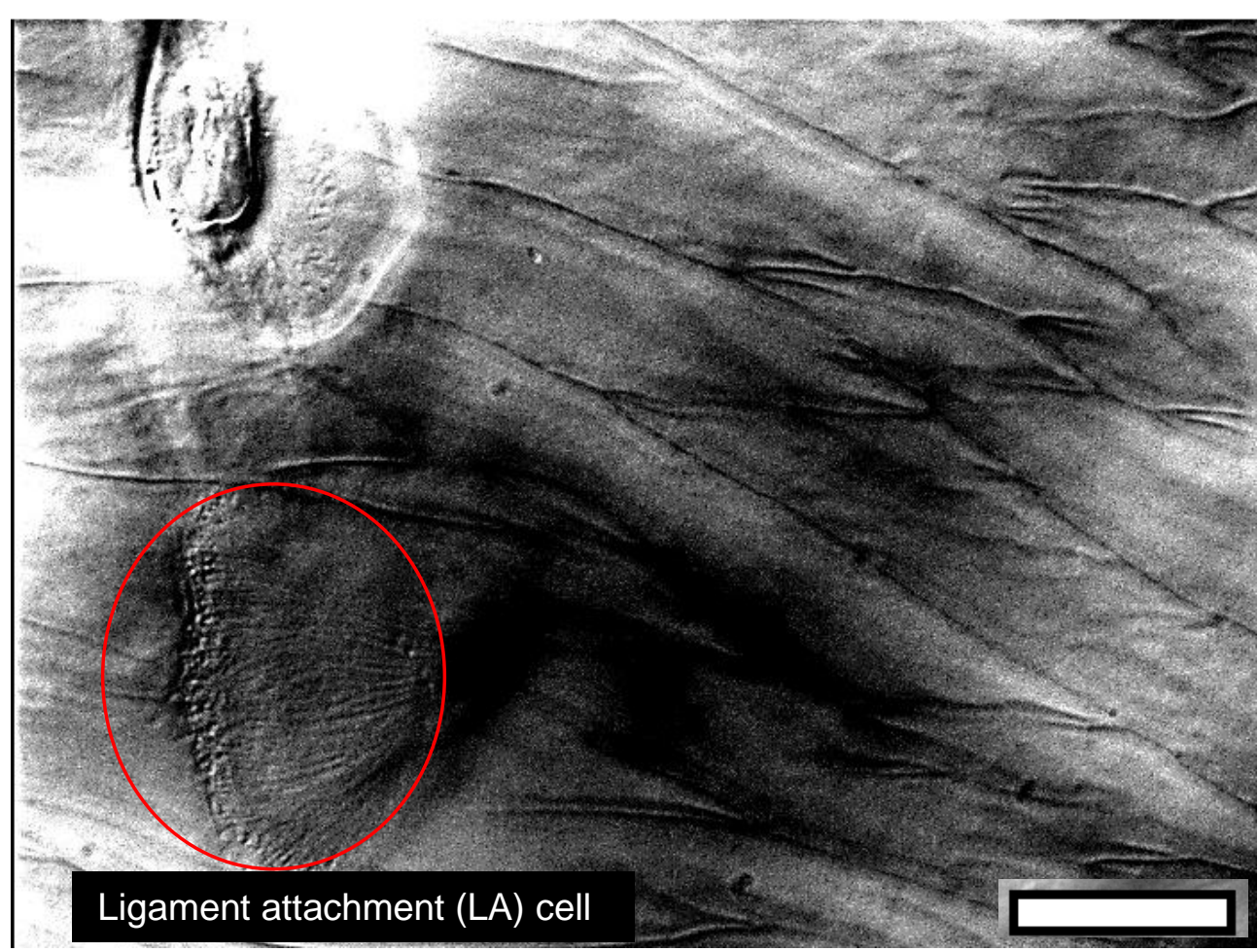

Fig. 4.3.1. The ligament attachment cell of the Ich5 organ. Scale bar $=15 \mu \mathrm{m}$. 

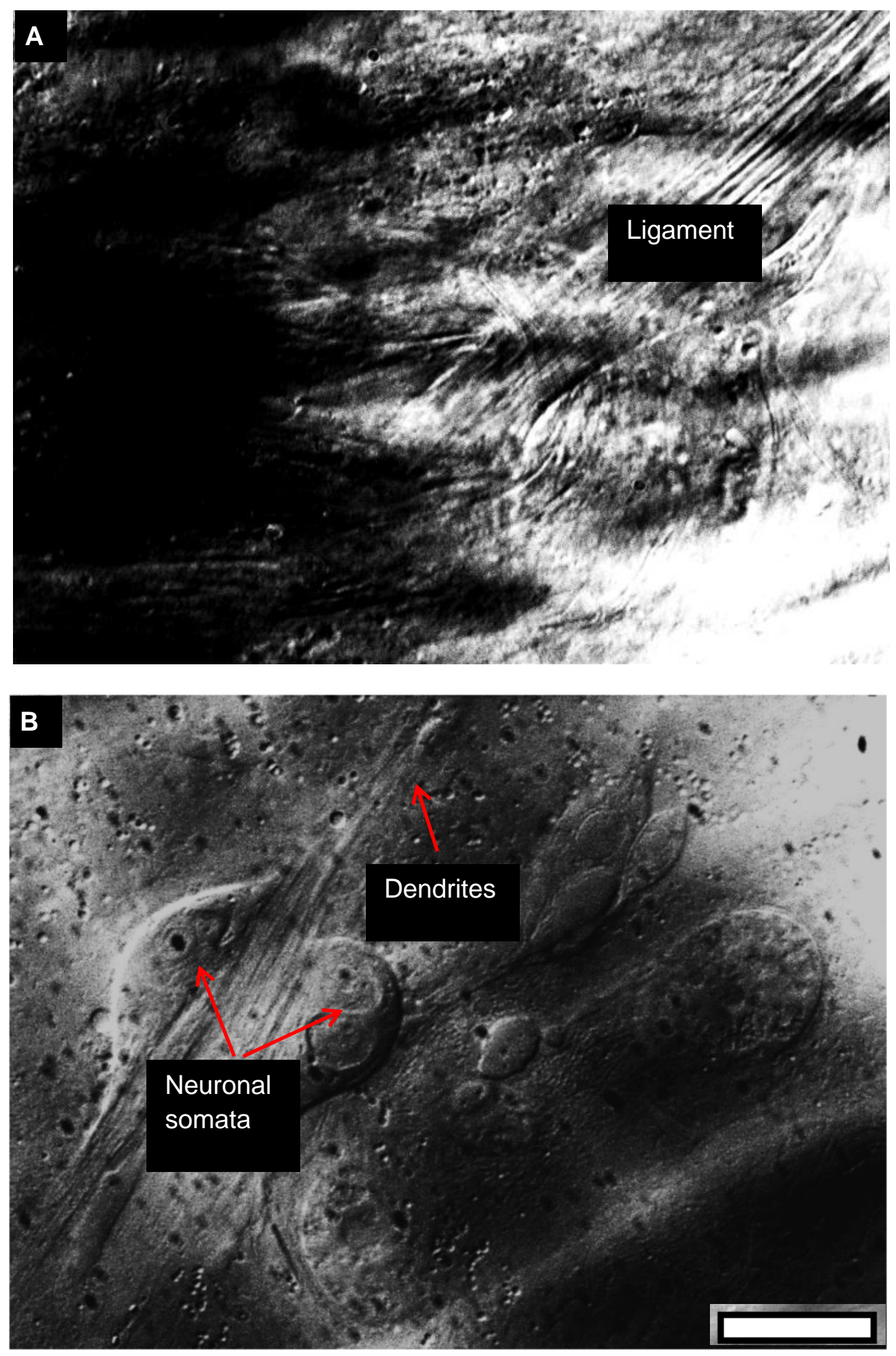

Fig. 4.3.2. (A) The ligament cells of the Ich5 organ (B) The ligament flanked by the neuronal somata, with the dendrites emerging from them. Scale bar $=15 \mu \mathrm{m}$. 


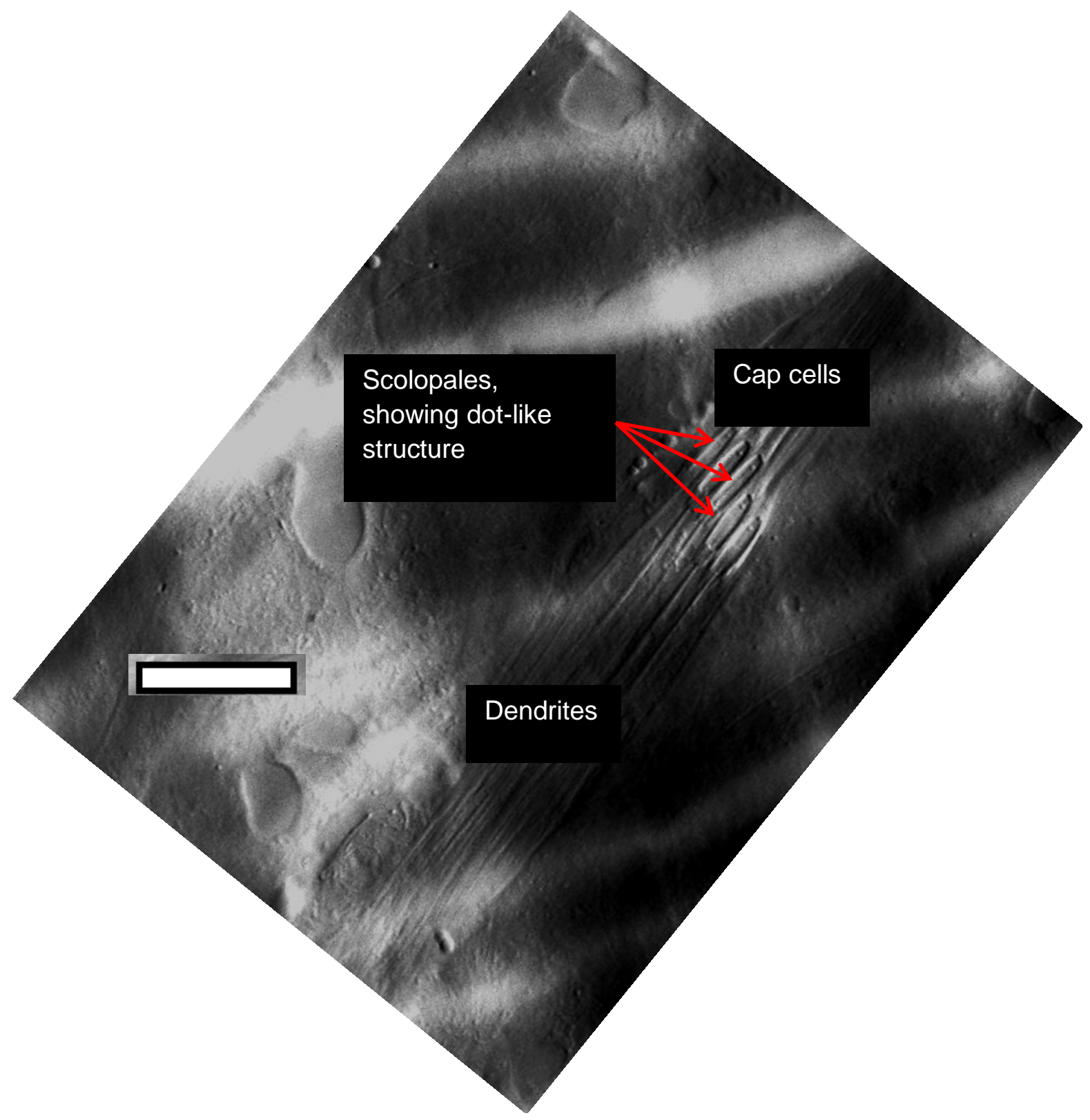

Fig. 4.3.3. The dendrites of the Ich5 neurons, covered in scolopale cells. At their apices, the scolopales are visible, as are the dot-like structures (most probably the ciliary dilations). The cap cells are seen to emerge from the apices of the scolopales. Scale bar $=15 \mu \mathrm{m}$. 

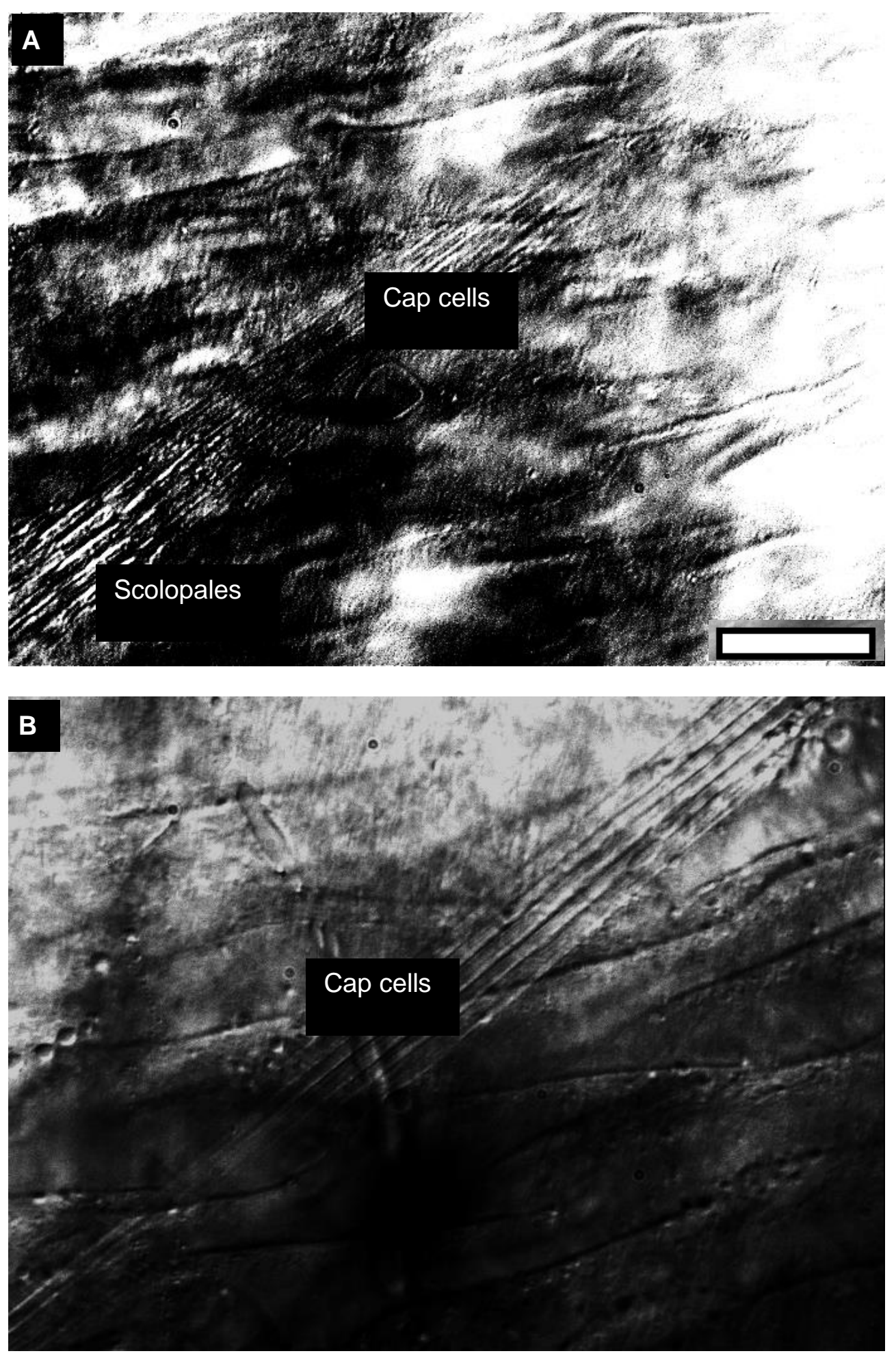

Fig. 4.3.4. (A) Scolopales and cap cells. (B) Cap cells alone. Scale bar $=15 \mu \mathrm{m}$. 

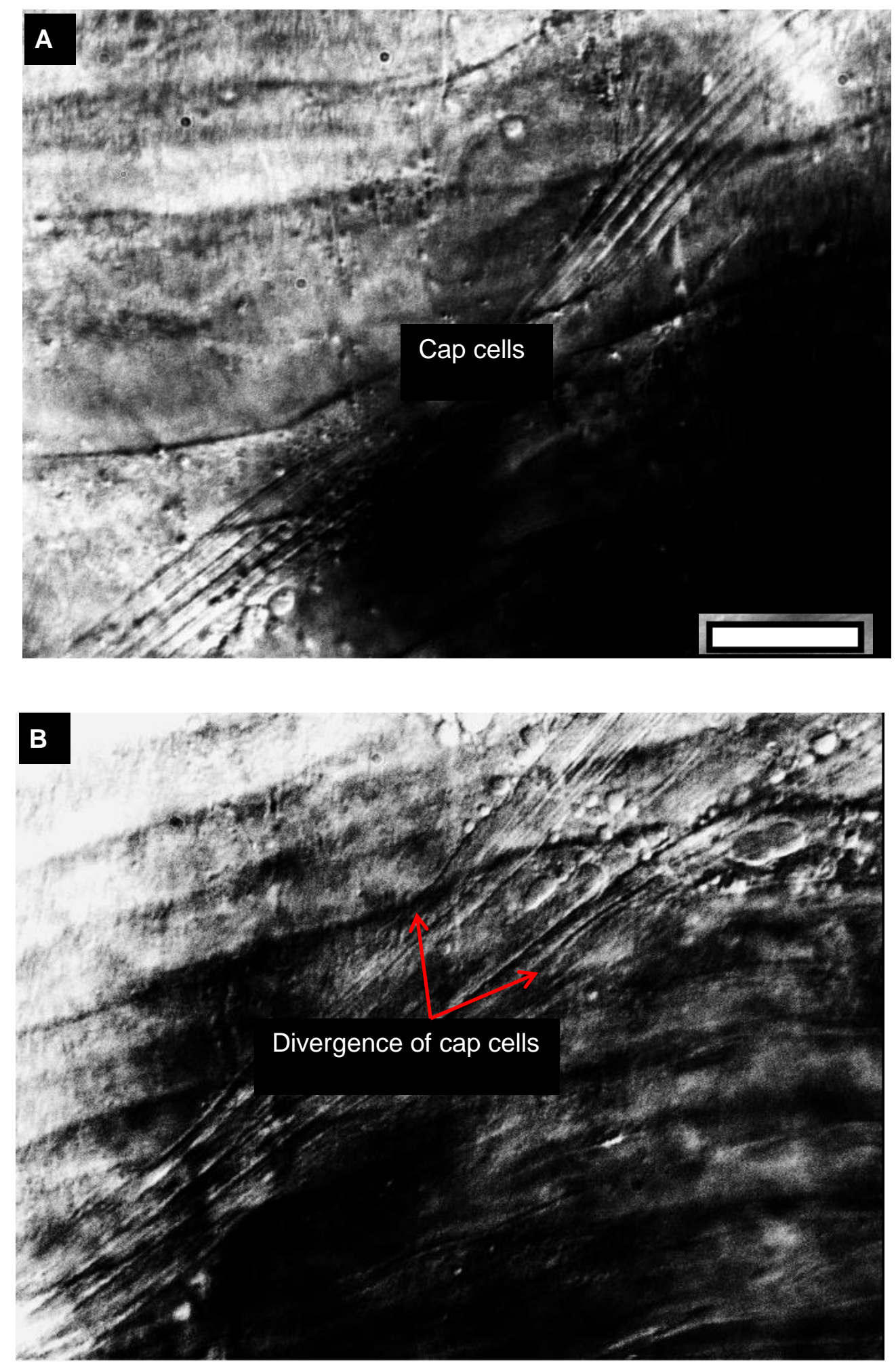

Fig. 4.3.5. (A) Distal part of cap cells. (B) Sometimes the cap cells are seen to split into two branches containing 2 and 3 cap cells respectively. 

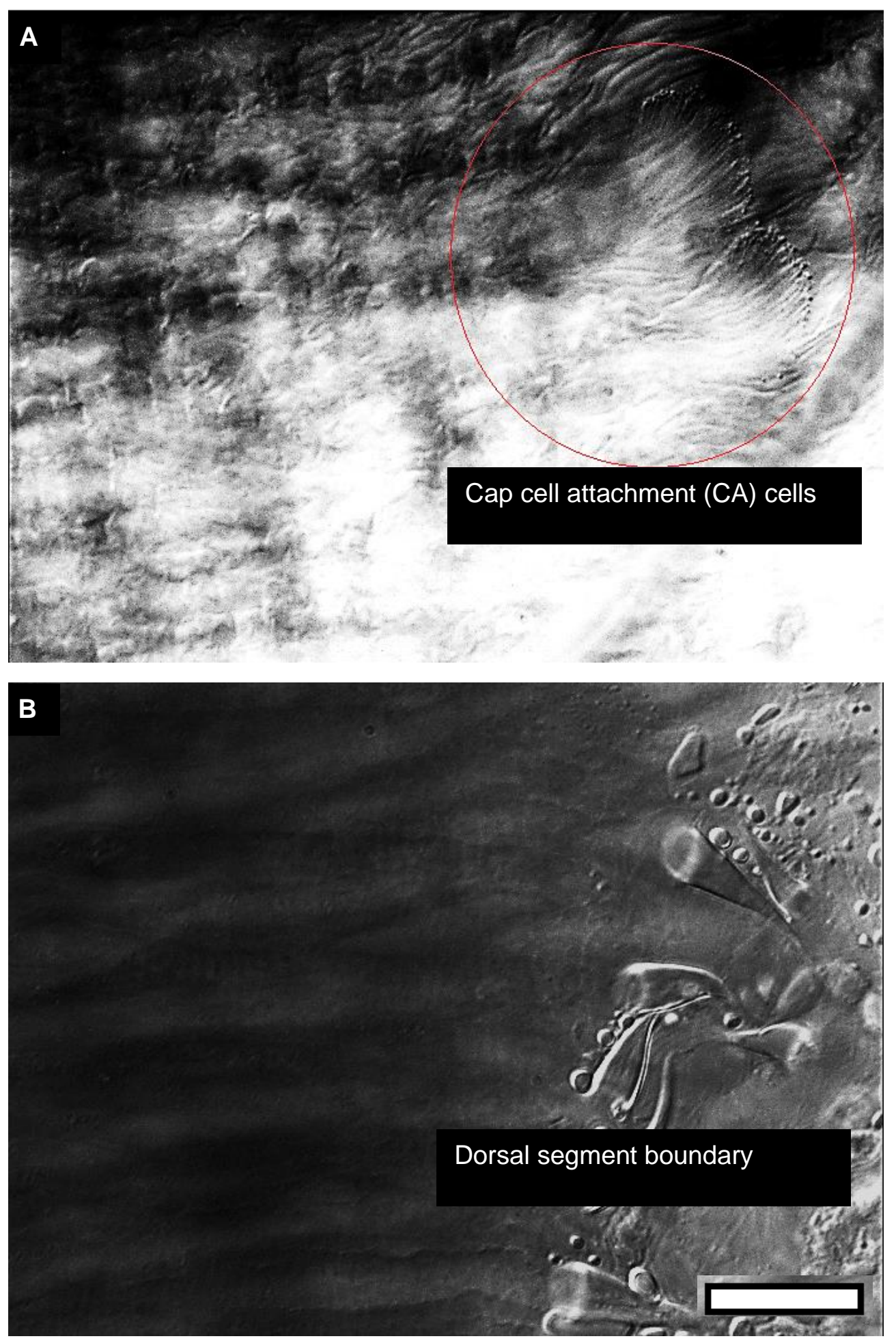

Fig. 4.3.6. (A) Cap cell attachment cells (2 in number). (B) Denticle-like structures seen at the segment boundary on the dorsal side. Scale bar $=15 \mu \mathrm{m}$. 


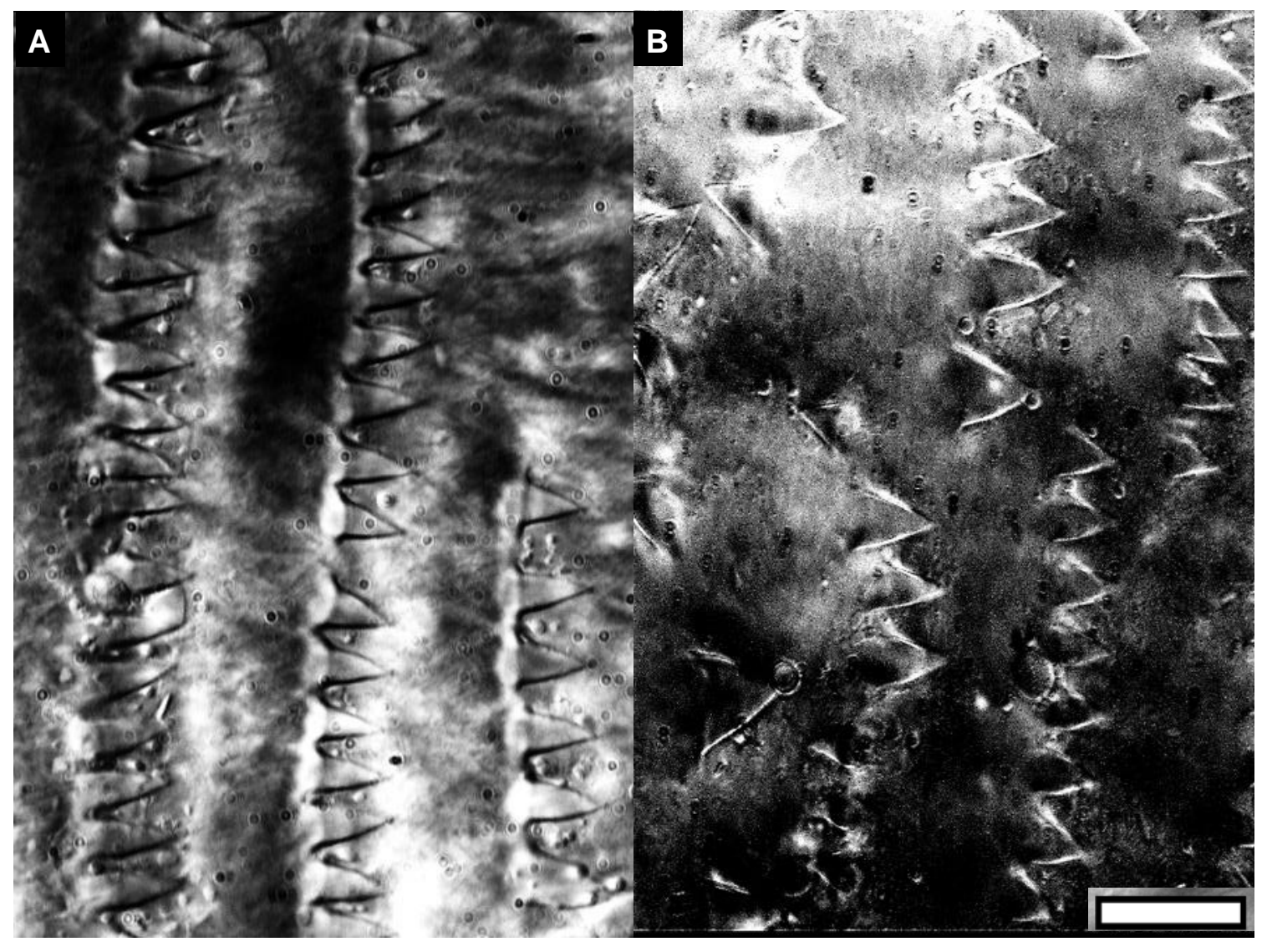

Fig. 4.3.7 (A) and (B). Denticle-like structures seen at the segment boundary on the dorsal side. Scale bar = $15 \mu \mathrm{m}$. 


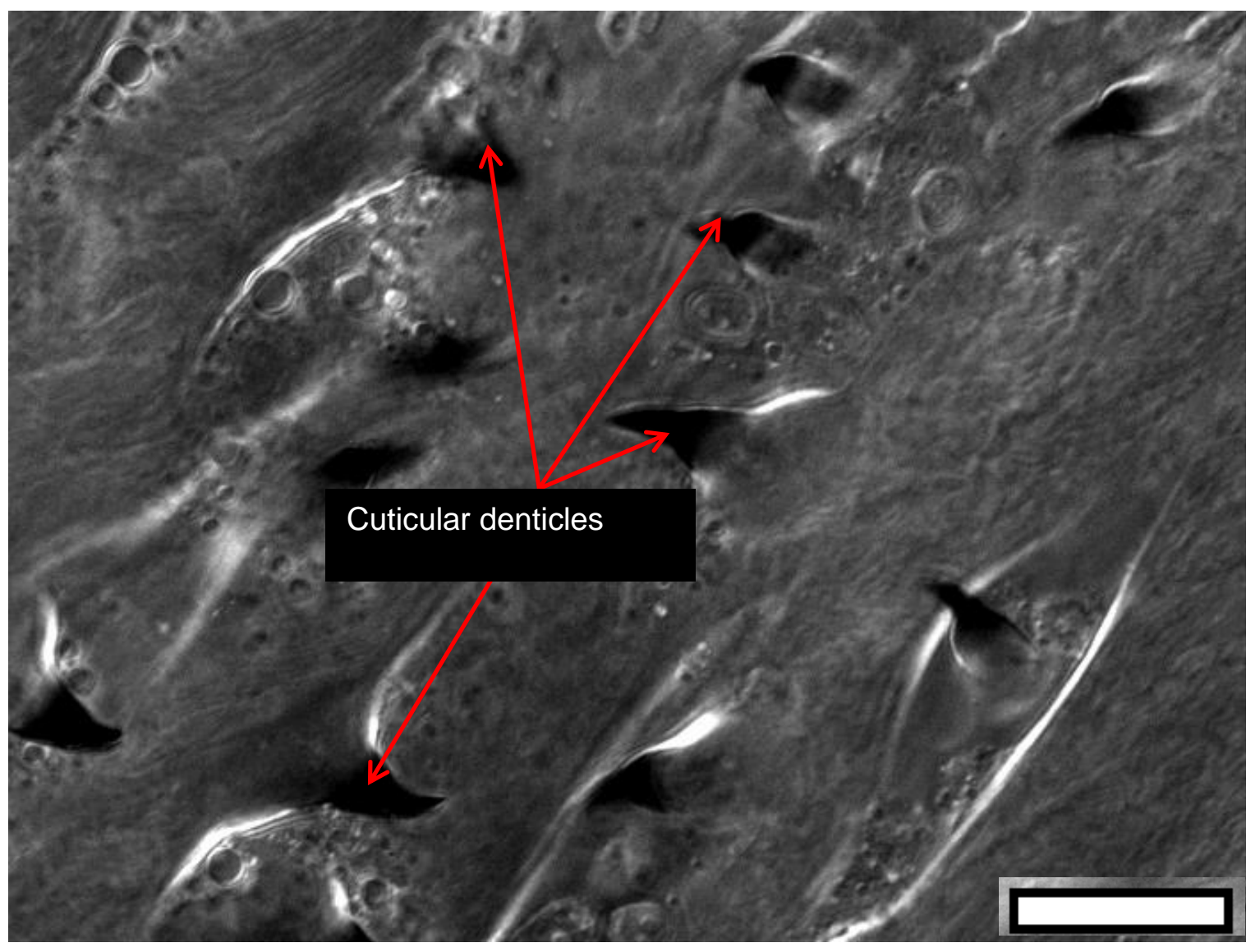

Fig. 4.3.8. Cuticular denticles on the ventral boundary (section 4.2). Scale bar $=15 \mu \mathrm{m}$. 


\section{Relaxation dynamics of the intact Ich5 organ}

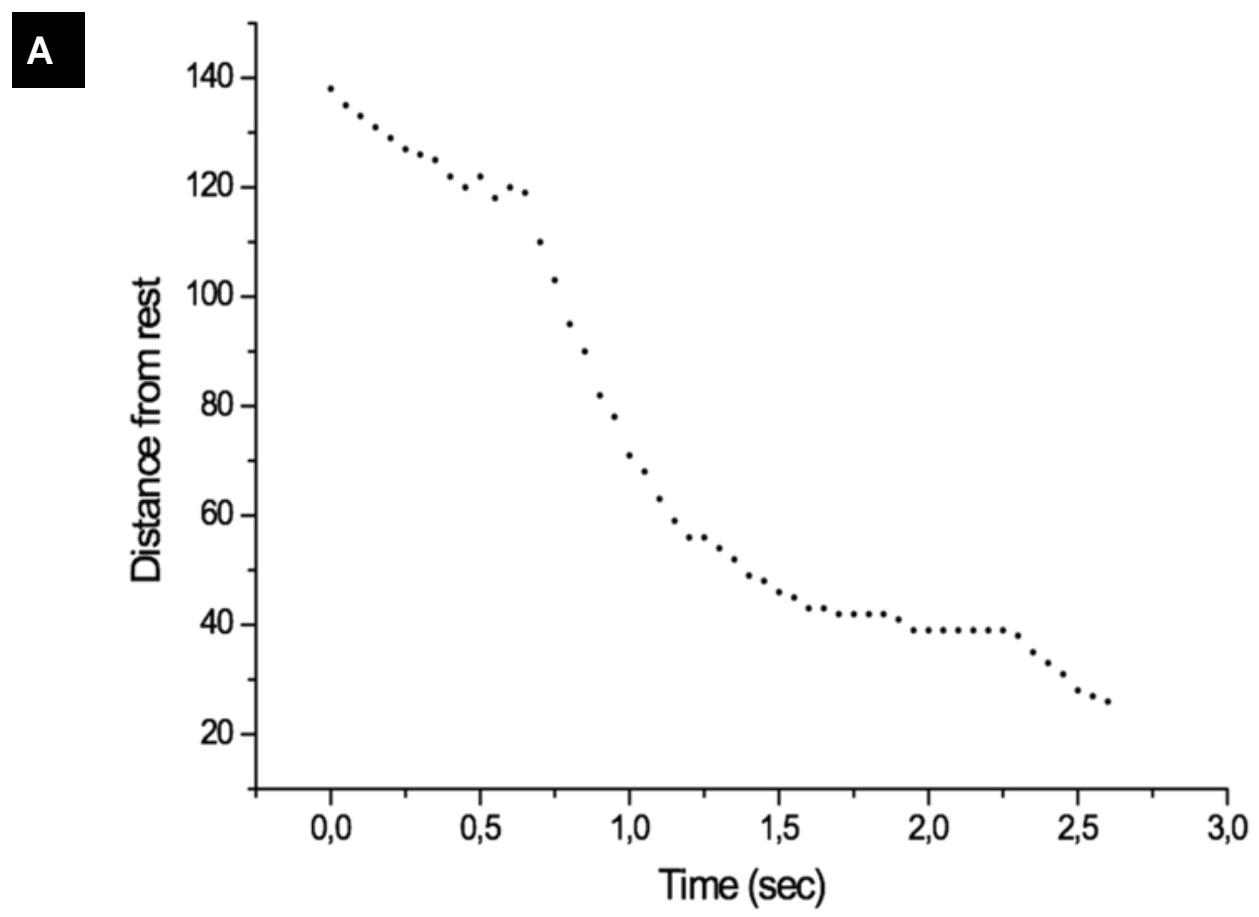

B

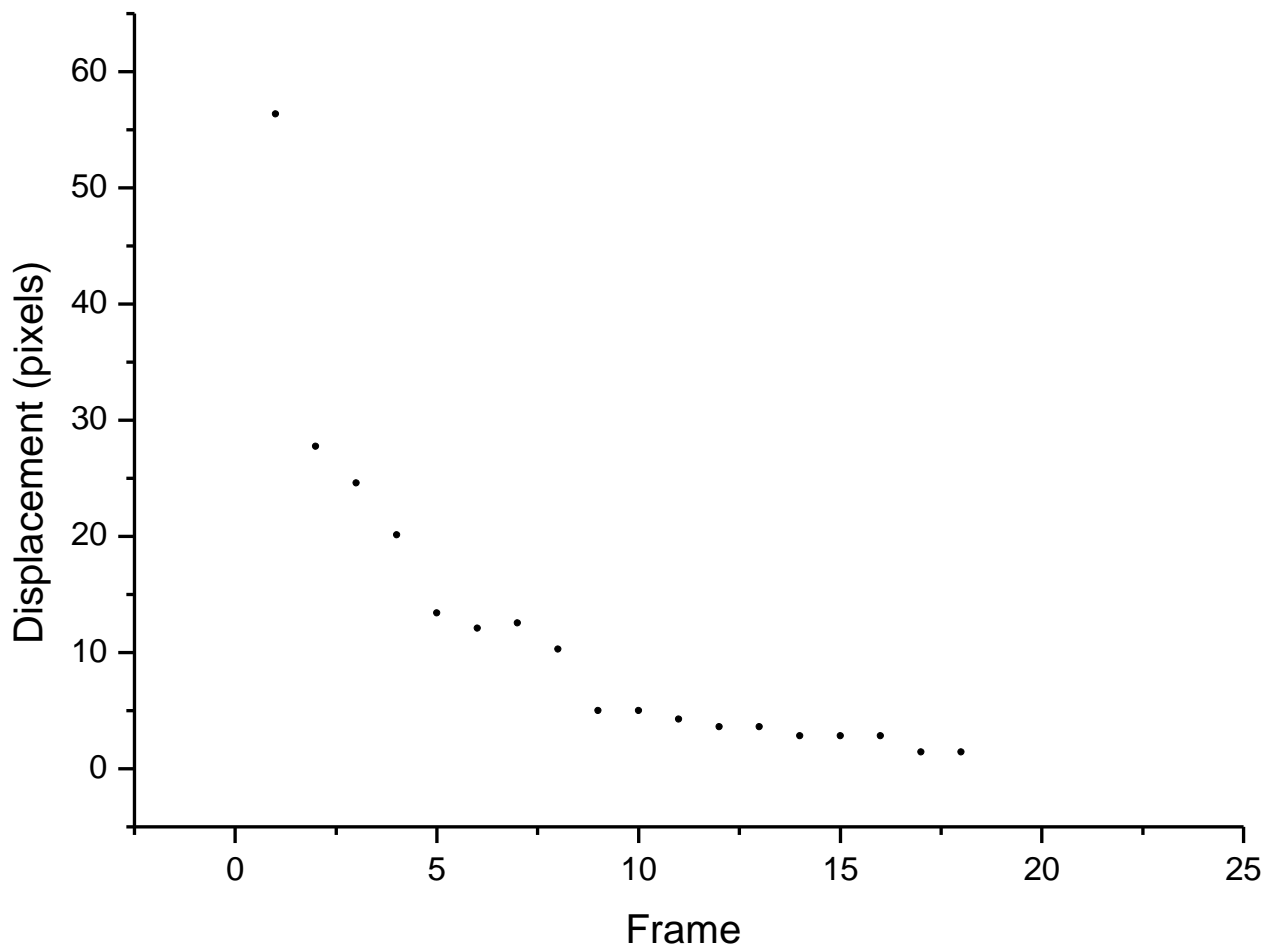

Fig. 4.4.1. (A) Incomplete disengagement of the Ich5 organ from the tungsten needle, indicated by a plateau in the graph at around $0.5 \mathrm{sec}$. Displacement values are in pixels. (B) Data points missing from the displacement vs time plot, owing to low frame rate of the camera. In this plot, displacement is in pixels, and the $\mathrm{X}$-axis represents the frame number of the video.

We next observed the relaxation dynamics of the deformed organ. I displaced the Ich5 organ perpendicular to its long axis with a tungsten needle placed against the cap cells adjacent to the 
scolopales. I then rapidly released the needle and tracked the relaxation of the organ to its original straight shape, in the form of displacement versus time plots. I fitted the data as an exponential curve. A fast snap-back and a slow relaxation were obtained (Fig. 4.4.1-4.4.3).

In our initial experiments, there were the following difficulties:

1. I was lifting the needle by manually rotating the dial of the manipulator. This meant that the needle was not immediately released from the Ich5 organ, resulting in a creep represented as a plateau on the displacement versus time plot (Fig. 4.4.1A). The exponential fit was inaccurate due to this reason. To counteract this problem, we changed the method. I shifted to using the "step" button on the micromanipulator, which rapidly lifts the pipette and needle by a finite distance. Sometimes, quickly pressing the button twice was required for complete release of the needle. When this was done, I did not observe the plateau anymore.

2. I was using the CoolSnap camera, with which one can achieve a maximum frame rate of only $22 \mathrm{fps}$. Due to this, many points on the displacement versus time plot were missing (Fig. 4.4.1B). The initial fast snap-back of the organ could not be captured at all. To solve this, we replaced the CoolSnap camera with the Photron FastCam which can capture images at a maximum of $1000 \mathrm{fps}$. I was then able to track the motion of the Ich5 organ during the fast snap-back.

3. Often I found that the needle was slipping against the muscles, because it was not properly bent at the tip. Producing a bent-tipped needle proved difficult. We eventually succeeded in producing such a needle, by using a combination of glass capillaries (section 3.8.3). I used the same needle for all subsequent experiments.

4. I was initially using $40 \mathrm{X}$ magnification. This meant that in the CoolSnap camera, only a small part of the organ could be observed (Fig. 4.4.2) in the relaxation experiments, since a small region of interest had to be chosen to make the frame rate higher. We then ordered a special 20X water-dipping objective from Zeiss, with which the entire organ became visible during the experiment, after rotating the sample so that it was parallel to the image plane. 

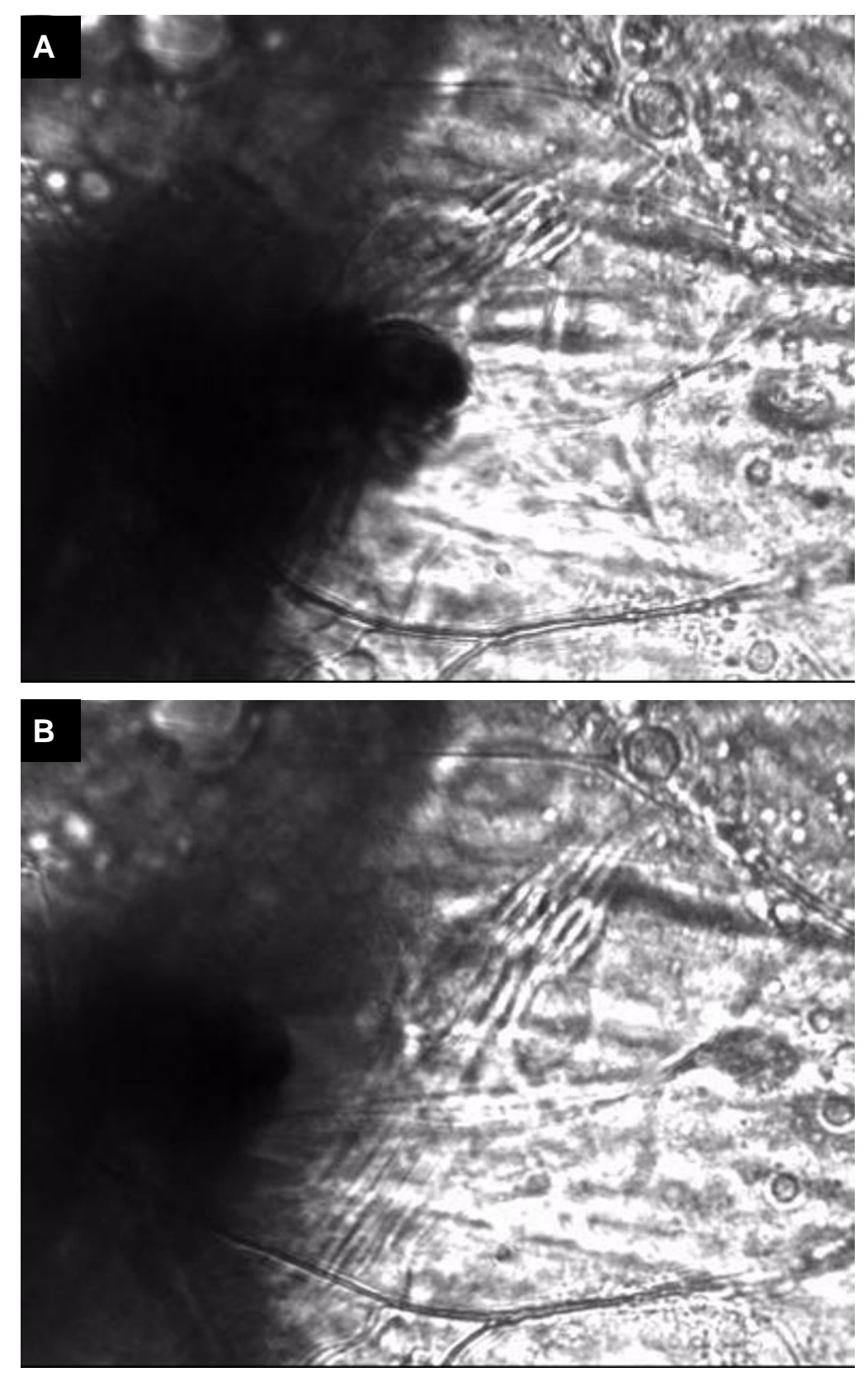

Fig. 4.4.2. View of the Ich5 organ under 40x magnification, (A) engaged with the tungsten needle, and (B) released from it. Only the dendrites and scolopales are visible, unlike in 20X where the entire organ is visible from the ligament attachment cells to the cap cell attachment cells.

5. We were using ivTrace to track the movement of the scolopales as they were released from the tungsten needle. ivTrace detects conspicuous structures and tracks their movement, and yields $X Y$ data. For this we needed to use larvae with GFP-fluorescent lch5 organs, from the fly line w[-];PBac[20XUAS-6XGFP]VK00018;iav-GAL4,w[+]/+. However the contrast was too poor when fluorescence was being used, in the sense that the organ was visible as a bright patch, but individual components could not be discerned (Fig. 4.4.3). We then reverted to 
using bright-field videos, and used the ImageJ Manual Tracking plug-in to track the scolopale movements manually.
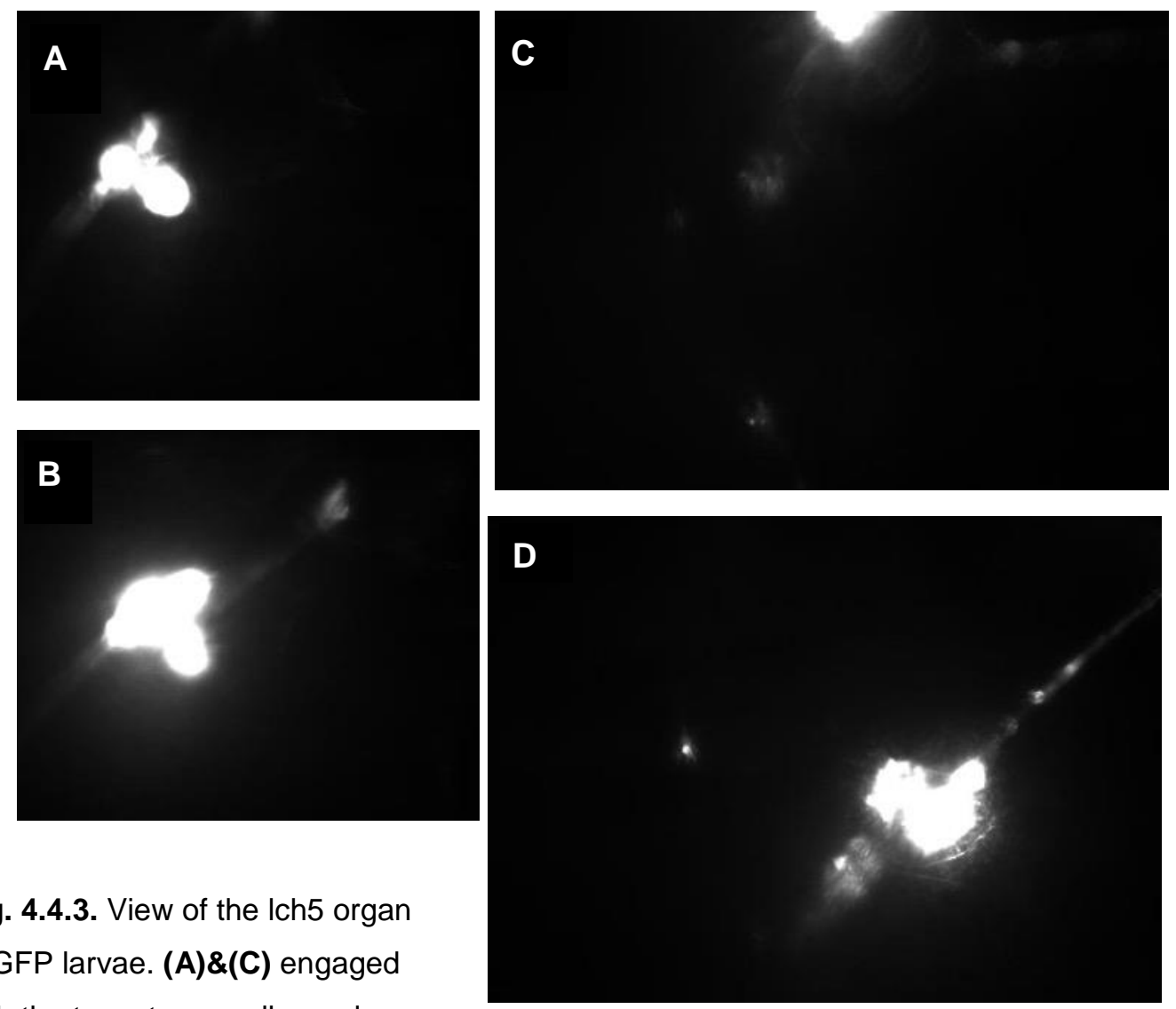

Fig. 4.4.3. View of the Ich5 organ in GFP larvae. (A)\&(C) engaged with the tungsten needle, and

(B)\&(D) released from it.

In fillet preparations with the muscle layers above the organ left intact, we observed an initial rapid snap-back after retraction of the needle, followed by a slow relaxation back to a straight conformation (Fig. 5.4.3 A and Fig. 5.4.4). The displacement time course was well fitted by a double exponential, which was verified by comparing a double-exponential fit with a single-exponential fit using Akaike's information criterion (168). In all cases - except for two recordings for one of the lch5 organs observed - the AIC value for the double exponential was lower, indicating that this was the better fit (Table 5.4.1). The two time constants obtained from this fit, $\tau_{1}$ and $\tau_{2}$, ranged from $10 \mathrm{~ms}$ to $150 \mathrm{~ms}$ for the initial fast relaxation, and $100 \mathrm{~ms}$ to $3 \mathrm{~s}$ for the slow relaxation (Fig. 5.4.3 C). In fillet preparations with the muscles excised, in contrast, a single exponential was sufficient to fit the data, because only a rapid snap-back was observed which was faster than in the preparations with intact muscles (Fig. 5.4.3 B, and Table 5.4.2). The time constants obtained in this case were about an order of magnitude smaller than the $\tau_{1}$ values for the fillets with intact muscles, and ranged from 3 
ms to $15 \mathrm{~ms}$ (Fig. 5.4.3 C and Fig. 5.4.5 A). The lch5 organs are embedded between muscle layers and the cuticle and bathed in haemolymph. In this situation, a rather high elastic stiffness would be needed to produce an underdamped or oscillatory response as has been speculated in the literature (74-76). Although the lateral deformation we applied with the tungsten needles is arguably nonphysiological, it is likely that the viscous damping in the system is similar to what occurs when muscles deform the cuticle and stretch the organ axially. The fact that we observe exponential relaxation and no sign of oscillatory response thus argues for overdamped dynamics. In contrast to a resonant response, an overdamped response would make the organ sensitive to perturbations over a broad frequency range, rather than only near a well-defined resonance frequency.

Most biological materials show a strongly non-linear elastic response $(169,170)$. To probe for nonlinear response of the Ich5 organs, we analyzed the dependence of the relaxation times on the amplitude of the lateral displacement. Within the margins of experimental error, we could not observe an amplitude dependence of these time constants (Fig. 5.4.3 D and Fig. 5.4.5 B). This means that nonlinearities are not strong enough to show clear effects, even at strains of $\sim 20 \%$. This is large compared to the strain for which actin filaments in typical cortical cytoskeletal networks become non-linear ( 5\%) (171) and might reflect a geometry of the elastic elements in the cap cells that is optimized for a broad linear response range.

Since the muscle tissue is arranged in layers above the lch5 organ, it might be possible that at least the outermost muscle layer (the lateral muscles) could directly attach to and activate the Ich5 organs. This possibility is already discounted in the literature $(71,72)$, but we nevertheless displaced the muscles with the tungsten needle to see if there was any direct coupling between muscle and Ich5 displacements. We could not discern any movement of the Ich5 organ directly elastically coupled to the movement of the muscles. Our data suggest, however, that there is a viscous coupling between the musculature and the lch5 organ, given that the relaxation dynamics are markedly slower in the presence of muscles than those of the bared lch5 organs. 
A

Displacement vs time plots: larval preparation with intact muscles
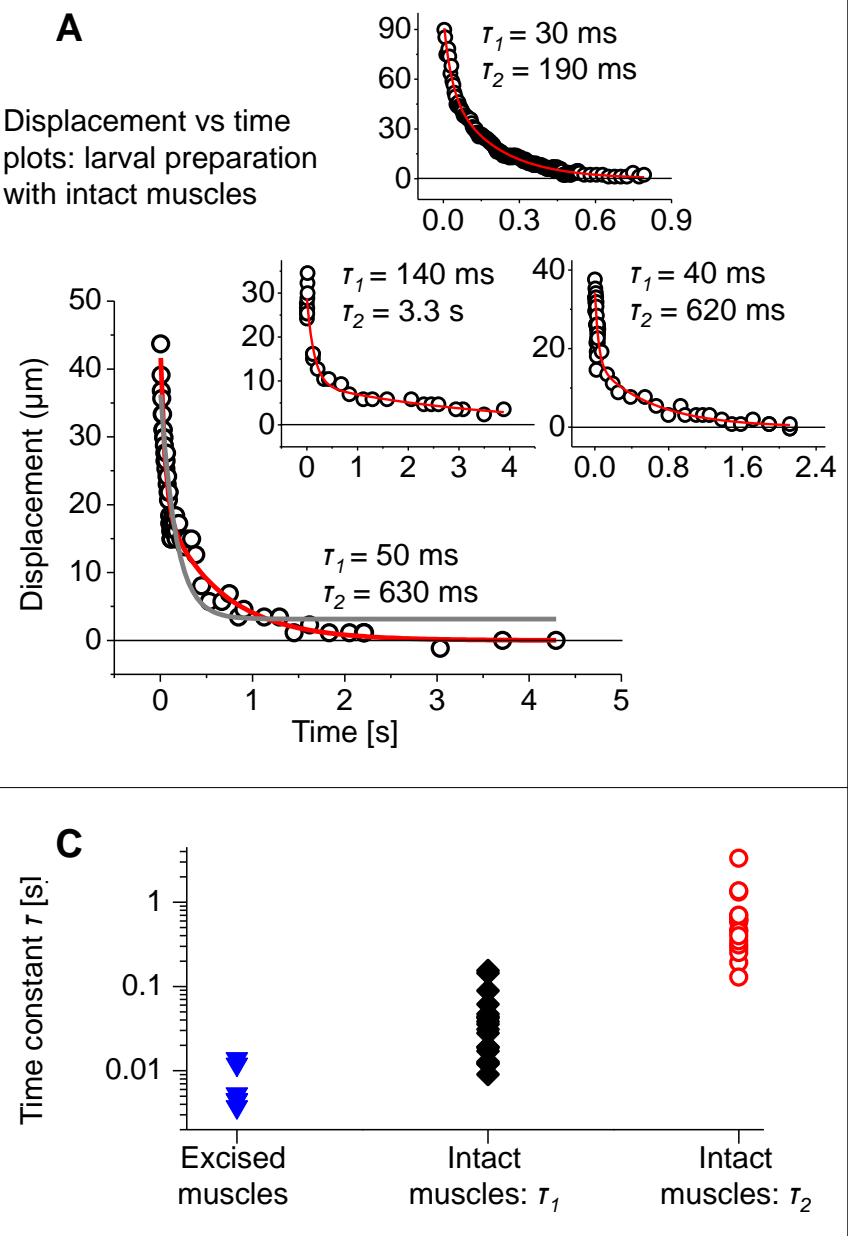

B

Displacement vs time plots: larval preparation with excised muscles
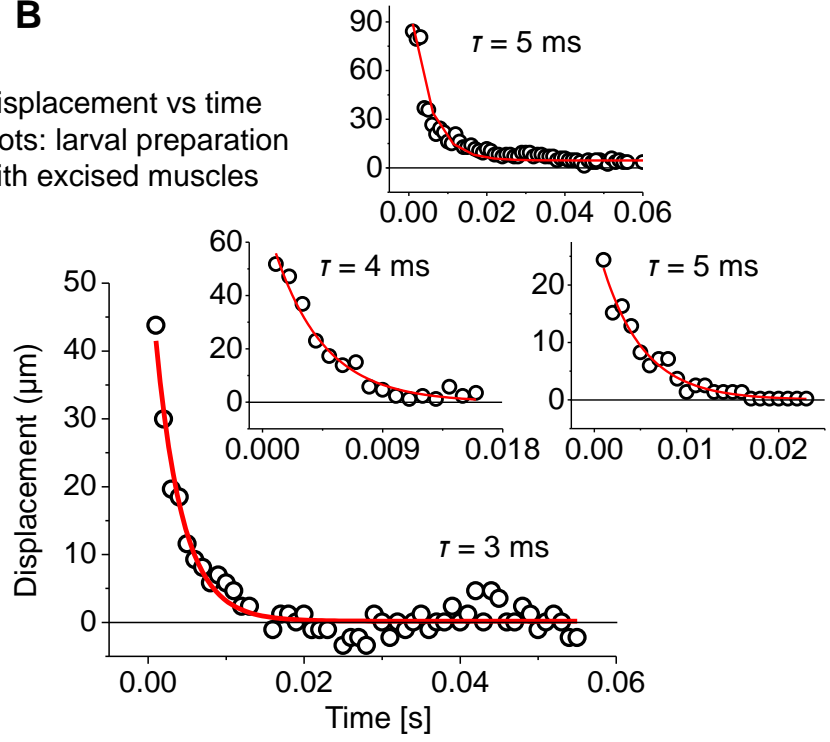

Fig. 4.4.4. Relaxation dynamics of the Ich5 organ. (A) Relaxation of the midpoint of the organ after lateral deflection and release with intact muscle layers. Each curve is for a different organ in a different animal. Red lines are doubleexponential fits with the two time constants $\tau_{1}$ and $\tau_{2}$ given. The grey line in the bottom graph is a single exponential fit. (B) Relaxation of the midpoint of the organ after lateral deflection and release with excised muscle layers. Red lines are single-exponential fits with the time constants $\tau$ given. The bottom and middle left plots are for the same Ich5 organ from one larva, and the top and middle right plots are for the same Ich5 organ from a different animal. (C) Relaxation time constants of Ich5 organs with the covering muscles excised (blue triangles), and for Ich5 organs covered by intact muscles, $T_{1}$ (black carets) and $T_{2}$ (red circles). Preparations with intact muscles: 4 animals studied. A total of 5 Ich5 organs were investigated. Animal \#1: 1 organ, 2 repeats. Animal\#2: 2 organs, 3 repeats each.

Animal \#3: 1 organ, 5 repeats. Animal \#4: 1 organ, 5 repeats. Preparations with excised muscles: 2 organs studied, each from a different animal, with 3 repeats per organ. (D) Amplitude dependence of Ich5 relaxation time constants with muscle layers intact (same experiments as the black and red plots in $\mathbf{C}$ ). 

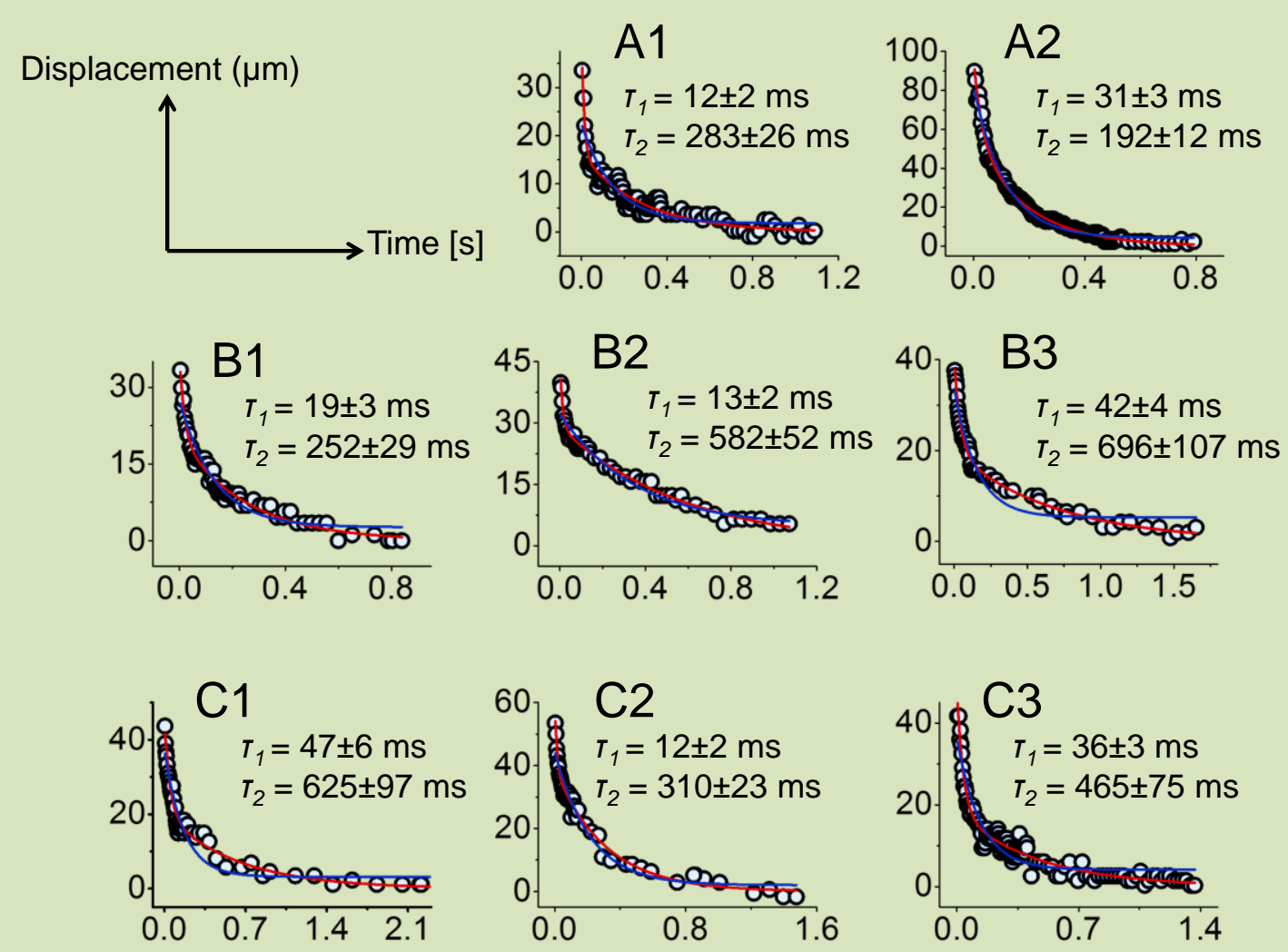

Displacement $(\mu \mathrm{m})$
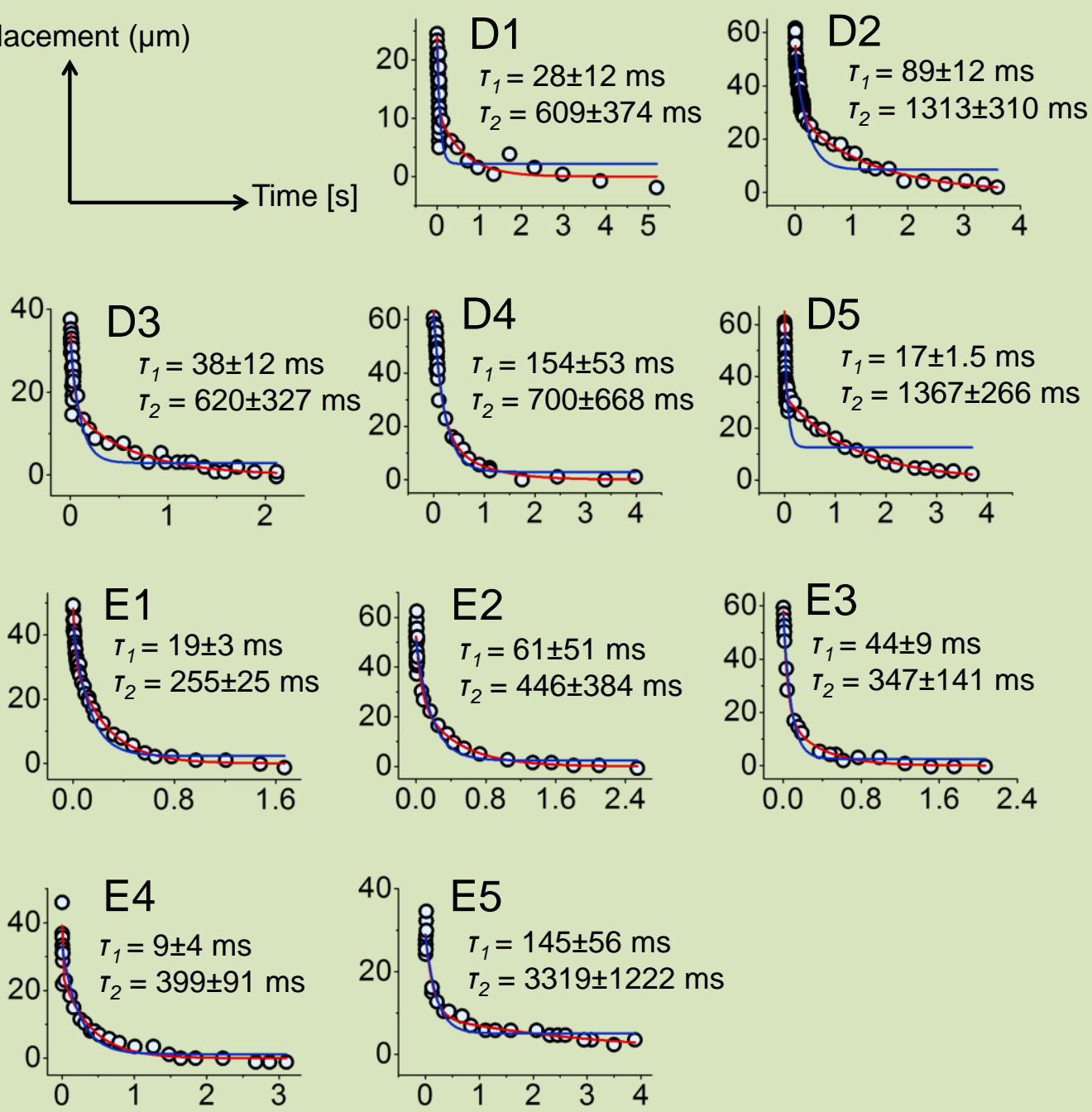
Fig. 4.4.5. Relaxation curves of laterally deflected Ich5 organs for all the relaxation experiments in larval preparations with intact muscles, showing double exponential fits (red curves) and single exponential fits (grey curves). Akaike information criterion (172) values for single and double exponential fits are given in Table 4.2.1 on the next page.

A1, A2: Animal 1, Ich5 organ 1

B1, B2, B3: Animal 2, Ich5 organ 1

C1, C2, C3: Animal 2, Ich5 organ 2

D1-D5: Animal 3, Ich5 organ 1

E1-E5: Animal 4, Ich5 organ 1 


\begin{tabular}{|c|c|c|c|c|}
\hline \multirow{2}{*}{$\begin{array}{l}\text { Experimental } \\
\text { animal }\end{array}$} & \multirow{2}{*}{$\begin{array}{l}\text { Ich5 } \\
\text { organ }\end{array}$} & \multirow[t]{2}{*}{ Trial } & \multicolumn{2}{|l|}{ AIC values } \\
\hline & & & Double exponential fit & Single exponential fit \\
\hline \multirow[t]{2}{*}{1} & \multirow[t]{2}{*}{ A } & A1 & 48.5 & 163.3 \\
\hline & & A2 & 96.4 & 255.4 \\
\hline \multirow[t]{6}{*}{2} & \multirow[t]{3}{*}{ B } & B1 & -4.2 & 67.6 \\
\hline & & B2 & -29.9 & 71.7 \\
\hline & & B3 & -18.0 & 89.9 \\
\hline & \multirow[t]{3}{*}{ C } & C1 & 50.1 & 116.6 \\
\hline & & C2 & 41.1 & 97.9 \\
\hline & & C3 & 105.3 & 261.2 \\
\hline \multirow[t]{5}{*}{3} & \multirow[t]{5}{*}{ D } & D1 & 160.0 & 165.3 \\
\hline & & D2 & 569.4 & 984.0 \\
\hline & & D3 & 136.8 & 149.2 \\
\hline & & D4 & 128.8 & 129.6 \\
\hline & & D5 & 153.9 & 280.0 \\
\hline \multirow[t]{5}{*}{4} & \multirow[t]{5}{*}{$\mathrm{E}$} & E1 & 71.5 & $\underline{70.3}$ \\
\hline & & E2 & 25.1 & 107.0 \\
\hline & & E3 & 148.9 & 147.8 \\
\hline & & E4 & 48.8 & 59.2 \\
\hline & & E5 & 183.3 & 307.3 \\
\hline
\end{tabular}

Table 4.4.1. Exponential fits of the relaxation curves of laterally deflected lch5 organs. Quality of fit is judged by the Akaike information criterion (172). AIC values for double and single exponential fits for the data plots from relaxation experiments for Ich5 organs in larval preparations with intact muscles. AIC values indicate the relative goodness of fit. The model that has a lower AIC value is considered as a better fit for the data. In all the cases except two, the double exponential model was seen to be the better fit. 


\begin{tabular}{|c|c|c|c|c|}
\hline \multirow{2}{*}{$\begin{array}{l}\text { Experimental } \\
\text { animal }\end{array}$} & \multirow{2}{*}{$\begin{array}{l}\text { Ich5 } \\
\text { organ }\end{array}$} & \multirow[t]{2}{*}{ Trial } & \multicolumn{2}{|l|}{ AIC values } \\
\hline & & & $\begin{array}{l}\text { Double } \\
\text { exponential } \\
\text { fit }\end{array}$ & $\begin{array}{l}\text { Single } \\
\text { exponential } \\
\text { fit }\end{array}$ \\
\hline \multirow[t]{3}{*}{1} & \multirow[t]{3}{*}{ W } & W1 & 19.2 & 16.6 \\
\hline & & W2 & 191.56 & 226.75 \\
\hline & & W3 & $\underline{40.3}$ & 121.7 \\
\hline \multirow[t]{3}{*}{2} & \multirow[t]{3}{*}{$x$} & $\mathrm{X} 1$ & 73.8 & 71.9 \\
\hline & & $\mathrm{X} 2$ & 54.4 & 44.7 \\
\hline & & X3 & 71.7 & 66.2 \\
\hline
\end{tabular}

Table 4.4.2. Exponential fits of the relaxation curves of laterally deflected Ich5 organs. Quality of fit is judge by the Akaike information criterion (172). AIC values for double and single exponential fits for the data plots from relaxation experiments for Ich5 organs in larval preparations with excised muscles. AIC values indicate the relative goodness of fit. The model that has a lower AIC value is considered as a better fit for the data. In all the cases except two, the single exponential model was seen to be the better fit. 

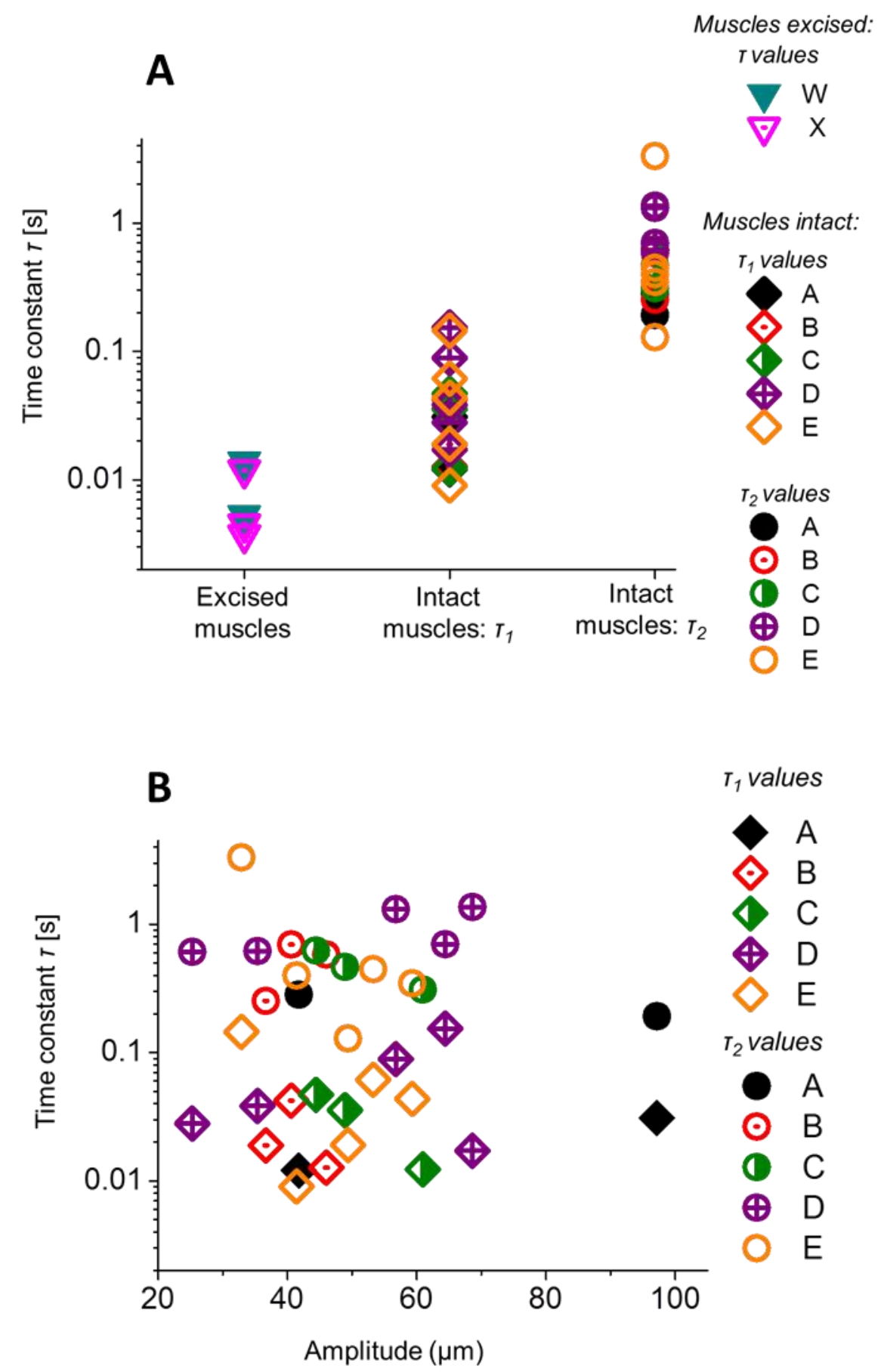

Fig. 4.4.6. Time constants for all relaxation experiments. $W$ and $X$ represent the larval preparations with muscles excised (time constants shown as inverted triangles), and $A, B, C, D, E$ refer to the experiments on preparations with intact muscles (see Fig. 4.4.5 and tables 4.4.1 and 4.4.2). In this case, diamonds represent the first time constant $\tau_{1}$ and circles represent the second time constant $\tau_{2}$. (A) Distribution of time constant values (refer Fig. 4.4.4 C). (B) Plot of time constants versus amplitude for the preparations with intact muscles (refer Fig. 4.4.4 D). 


\subsection{Static elastic deformation of the Ich5 organ}

We studied the static deformation of the Ich5 organ under stretching by a lateral force applied using a tungsten needle controlled by a micromanipulator (Fig. 4.5.1). The needle was placed near the midpoint of the organ, against the cap cells. From video recordings, we tracked the positions of the scolopales and of the two attachment cells to map the motion and deformation of the entire organ. Lateral displacement near the middle of the organ could have different effects on the organ and its embedding structures: If the organ itself were rather rigid to axial stretching, the cuticle could deform, with the attachment cells moving inward to accommodate this deformation. Alternatively, or in addition, the different cells making up the organ could stretch to various degrees, increasing the length of the organ. In all experiments in which we observed the attachment cells, we could not detect any displacement of the cuticle and the attachment cells when we deformed the lch5 organs (Fig. 4.5.1). The organ itself is therefore distinctly more stretch compliant than the cuticle is to bending and indentation.

If we consider a third instar larva as a cylinder of diameter $1 \mathrm{~mm}$, then given that the lch5 organ can be upto $400 \mu \mathrm{m}$ long in the fillet, we obtain only a $3 \%$ increase in the length of the lch5 organ from the intact larva to the fillet. For details, see Appendix A1.

When the Ich5 organ was displaced by the tungsten needle, we measured a substantial, approximately $10 \%$ increase in the length of the organ (from $\sim 320$ to $\sim 350 \mu \mathrm{m}$ for a lateral deflection of $80 \mu \mathrm{m}$ ). This stretch was reversible and the organs relaxed back to their original lengths when the needle was released (Figs. 4.5.1-2). The length increase was almost entirely confined to the cap cells. This is demonstrated by tracking 4 points: the end points of the ligament (LA) and the cap cells (CA), the point of contact of the needle with the lch5 organ (T) and the position of the scolopales (S). We define the axis of the chordotonal organ - the line from CA to LA - as the X-axis (Fig. 2D). The portion from $S$ to CA corresponds to the cap cells. The length from CA to LA ranged between 300 and $400 \mu \mathrm{m}$. The portion between LA and S, i.e. the part of the organ consisting of the ligaments, neuronal somata, dendrites and scolopales, had a length between 90 and $100 \mu \mathrm{m}$ before lateral deflection. We observed that during stretches, LA and CA remained fixed (no detectable movement along the $X$ or $Y$ axes), while $T$ moved along a straight line parallel to the $Y$-axis, further away from the position of rest. The length from LA to $S$ also slightly increased, but much less than the length of the cap cells. The cap cells reportedly contain more myosin motors and actin filaments than the sensory neurons, and the scolopales lack myosin while actin is present (124-126). Myosins interacting with actin filaments might provide a mechanism to tense the cap cells and to create stretch elasticity to allow for substantial elongation and subsequent recovery. The observation that the 
largest part of the elastic strain in the stretched organ occurs in the cap cells implies that they might play an important role in the stretch-sensing mechanism and might be in charge of setting the prestrain and providing adaptation. The relative length increase of the lch5 organ as a function of the relative lateral displacement was reproducible between several organs from different larvae (Fig. 4.5.2 B).

The shape of the deformed organ allows us to draw qualitative conclusions about the elastic properties of the organ and of the embedding tissue. In the most stretched conformations, the Ich5 organ assumed a concave cusp-like shape in the cases where the muscle layers were not removed (Fig. 4.5.1 B\&C). This implies that the bending stiffness of the organ is low compared to the stretching stiffness. The shape of the organ would be convex if resistance to bending was dominant. Furthermore, the fact that we observe concave rather than straight shapes points to some coupling to the elastic embedding tissue layers (173). 

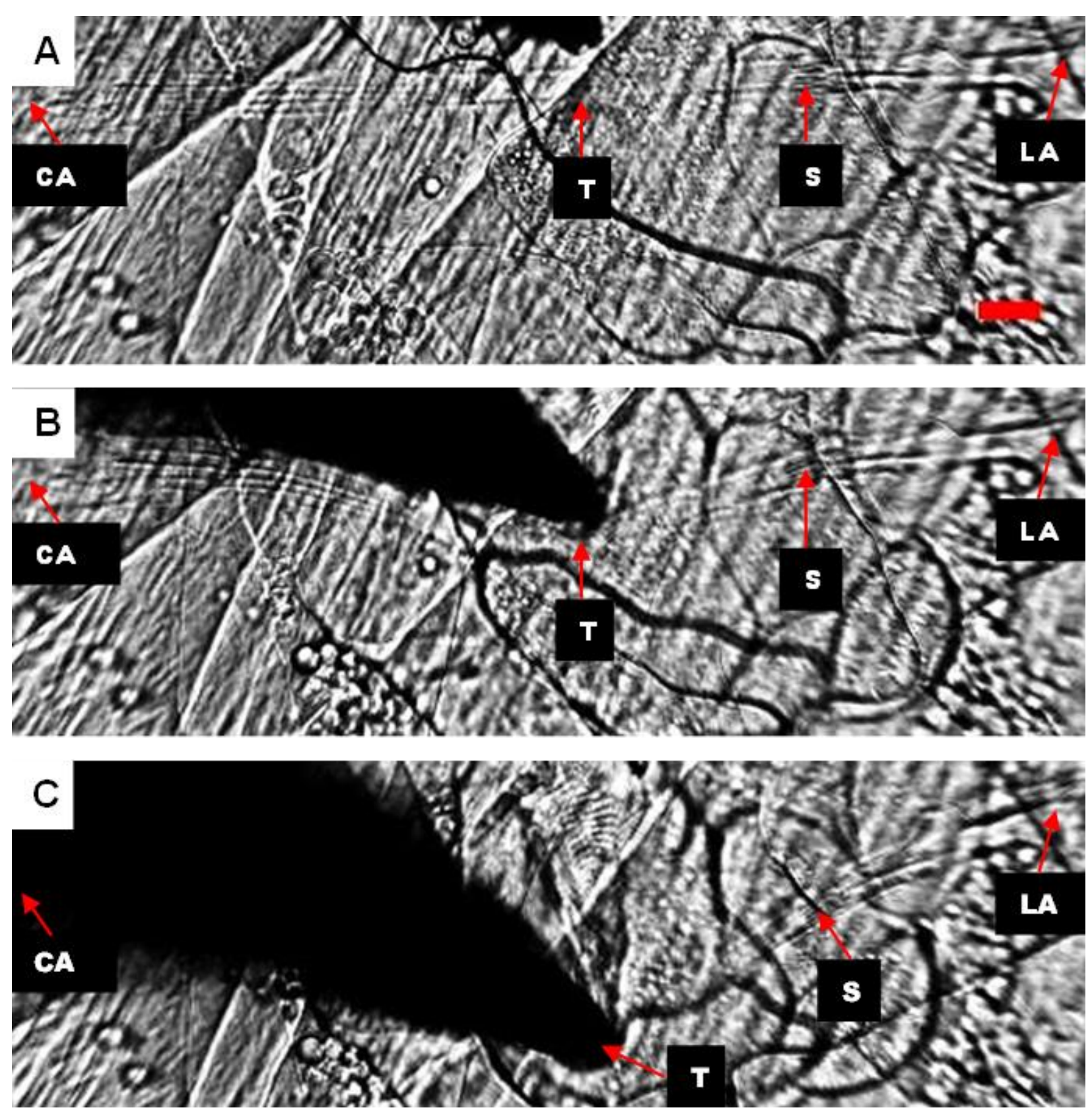

Fig. 4.5.1. Lateral deflection of the Ich5 organ. (A) Relaxed, (B) weakly deflected ( $34 \mu \mathrm{m}$ ), and (C) strongly deflected $(86 \mu \mathrm{m})$ configurations. The dark tip is the tungsten needle. The organ is oriented parallel to the long side of the image (neuronal cell bodies on the right). Scale bar: $20 \mu \mathrm{m}$. CA: attachment cell for cap cells, LA: ligament attachment cell, S: scolopales, T: point of contact of needle and Ich5 organ. 


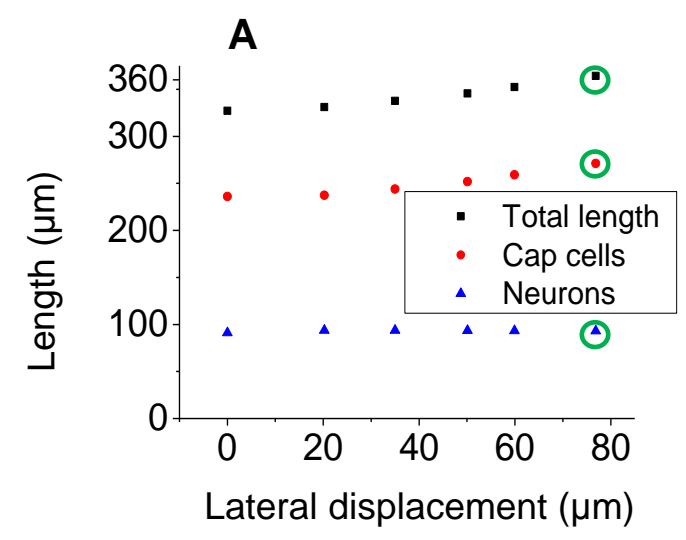

B

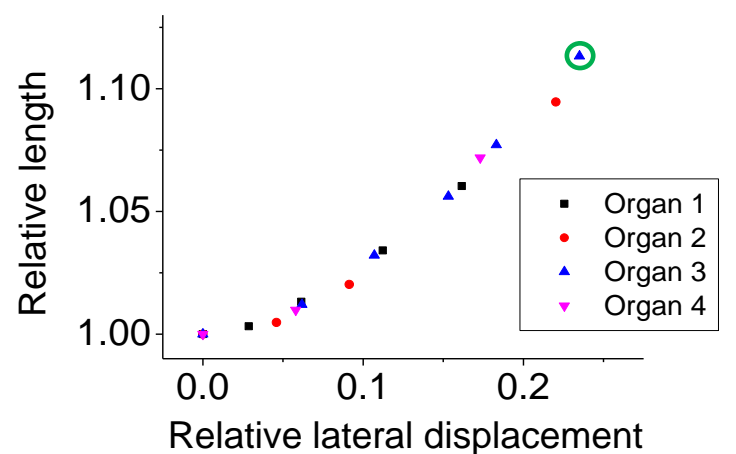

C

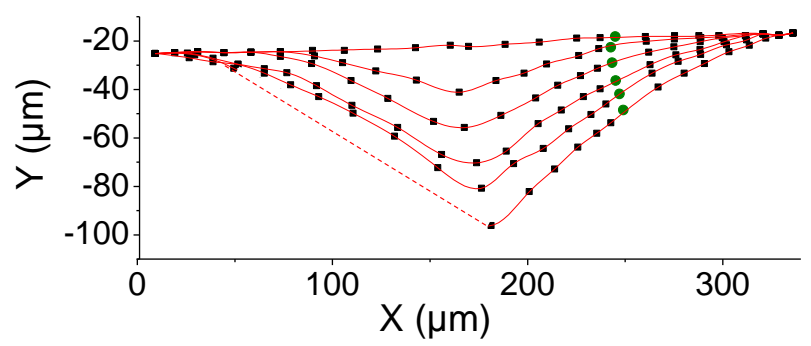

- Track points (extension from original length
- Scolopale position
- Track points (return to original length)
Cubic spline interpolation (extension)
Cubic spline interpolation (return)

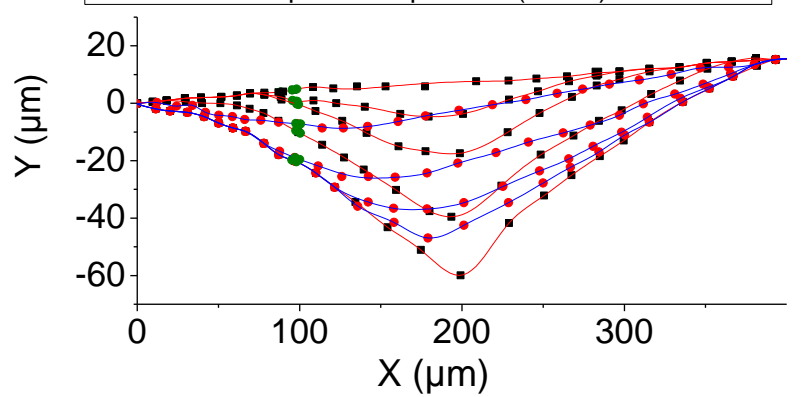

Fig. 4.5.2. Static elastic deformation of the Ich5 organ. (A) Increase of total length with lateral deflection, entire organ (black squares), cap cells (red circles) and neuronal part (blue triangles). (B) Relative increase in length versus relative lateral deflection amplitude for 4 different lch5 organs, each from a different larva. (C) Shapes of a representative Ich5 organ (same as Fig. 4.5.1) traced from a video recording while the organ was increasingly deflected. (D) Shapes of another representative Ich5 organ traced from a video recording while the organ was increasingly deflected (black squares and red cubic spline interpolation line) and during relaxation (red circles and blue cubic spline interpolation line).

In (C), part of the last curve has been shown as a dotted line, since this part of the organ was obscured by the needle in the frame of the video corresponding to this curve. The last points in $(A)$ and $(B)$ are encircled in green for the same reason, and represent a lower bound for the value of the length of the organ at that point. 


\subsection{Relaxation of the Ich5 organ after laser ablation}

For these experiments, I did not use the fillet preparation, because the imaging had to be carried out using slides and coverslips. Instead, I used the "squished" prep described in section 3.6, where the entire larva was squeezed between a slide and a coverslip. The gut was removed, and the larva was rendered transparent. We carried out most of the experiments using fluorescence, but even in bright field the lch5 organs were clearly visible.

The straight conformation of the Ich5 organ, appearing like a bundle of tense chords (which also inspired the name "chordotonal organs" (74-76) suggests that there is substantial axial pre-tension in the cells making up the organ. Tension is balanced along the whole organ, but the ensuing elastic strain might be different for the different cell types. To characterize the pre-strain in the different components of intact Ich5 organs, we used a UV laser to cut the organ in different planes - through the cap cells near the scolopales (Fig. 4.6.1 A and Fig. 4.6.2), through the ligament cells (Fig. 4.6.1 $\mathrm{B}$ ), and through the dendrites (Fig. 4.6.1 D and Fig. 4.6.3). The response of the organ was observed via the displacement of the GFP-expressing neurons (fly line: $w[-] ; P B a c[20 X U A S-$ 6XGFP]VK00018;iav-GAL4,w[+]/+) in a fluorescence microscope. We cut the cap cells one by one, by slowly moving the laser focus across the organ (Fig 4.6.2). When the cap cells were completely severed, the scolopales and neurons retracted towards the ligament cells by about $20 \mu \mathrm{m}$ (Figs. 4.6.1 A \& 4.6.2 A\&D), indicating a resting strain of $\sim 20-25 \%$ in this section of the organ. The neuronal somata did not deform noticeably, which indicates that the axons anchoring the somata in the surrounding tissue also relax with motion toward the ligament side of the organ, as shown in the sketch by the angled attachment of the axons (Fig. 1). The severed dendrite retracted and a kink formed where the dendrite enters the scolopale (Fig. 4.6.2). With each cap cell cut, the neurons retracted further. When the ligament cells were severed after cutting the cap cells, we observed a slow retraction of the neurons towards the cap cells by $\sim 30 \mu \mathrm{m}$ (Fig. 4.6.1 B). In the case where the ligament cells were severed leaving the cap cells intact, the neuronal somata were still anchored by their axons, and there was practically no retraction (Fig. 4.6.1 C). However, when the axons were severed, there was rapid retraction in the direction of the cap cells (section 7.3 in the appendix). When, in the third group of cutting experiments, the dendrites were severed, we could observe the relaxation of both sides of the organ by following the retracting fluorescent neuronal somata towards the ligament cell side and the retracting fluorescent dendritic fragments towards the cap cell side. We performed two types of cutting experiments, now cutting the dendrites all at once (Fig. 4.6.1 D), or one by one (Fig. 4.6.3). Either way, when the dendrites were all cut, the severed dendritic fragments, and the scolopales along with the cap cells retracted rapidly by $\sim 100 \mu \mathrm{m}-$ corresponding to a pre-strain of $\sim 50 \%$ in this section of the Ich5 organ. When the cap cells were cut, the dendrites and scolopales also retracted by about $30 \%$ of their initial length, but the neuronal somata remained 
more or less stationary. This points to a role of the cap cells as the dominant elastic elements in the Ich5 organs, consistent with what we found when we overstretched the organ by lateral deflection, where the additional strain was again mostly localized to the cap cells. Since the cap cells also contain substantial amounts of myosin and actin, it is intriguing to speculate that these cells build up - and regulate - organ tension.

We also carried out similar experiments on 2 other fly lines: Sqh-GFP (myosin labelled with GFP in cap cells and ligaments), and UAS Sqh-RNAi X Pinta GAL4 (myosin knockdown mutant). In SqhGFP we were mainly observing the retraction of the cap cells - which was not visible in the earlier case since in that fly line it was only the neurons that were fluorescent. Of course, when we severed the dendrites, the cap cell retraction was indicated by the movement of the scolopales, which were labelled. But in Sqh-GFP larvae we could directly observe the motion of the cap cells. Here we observed slightly less retraction ( $~ 80 \mu \mathrm{m}$ as opposed to $\sim 100 \mu \mathrm{m})$ (Fig. 4.6.3). It is possible that the labelling has a slight effect on the functioning of the myosin. In the knockdown mutant (Fig. 4.6.5 B), the retraction was much smaller $(\sim 50 \mu \mathrm{m})$ as compared to the control $\mathbf{w}^{118} \mathrm{X}$ Pinta GAL4 (Fig. 4.6 .5 A), which is a very interesting observation. These results correlate well with our hypothesis that myosins in the cap cells must play the dominant role in mechanical regulation. It is worth noting that while these last two fly lines exhibit no fluorescence, it is still possible to conduct ablation experiments on them. The only disadvantage is that the apparatus settings make it not possible to take very long videos if one does not use fluorescence, but this can at least be partially overcome by taking "before" and "after" images. 

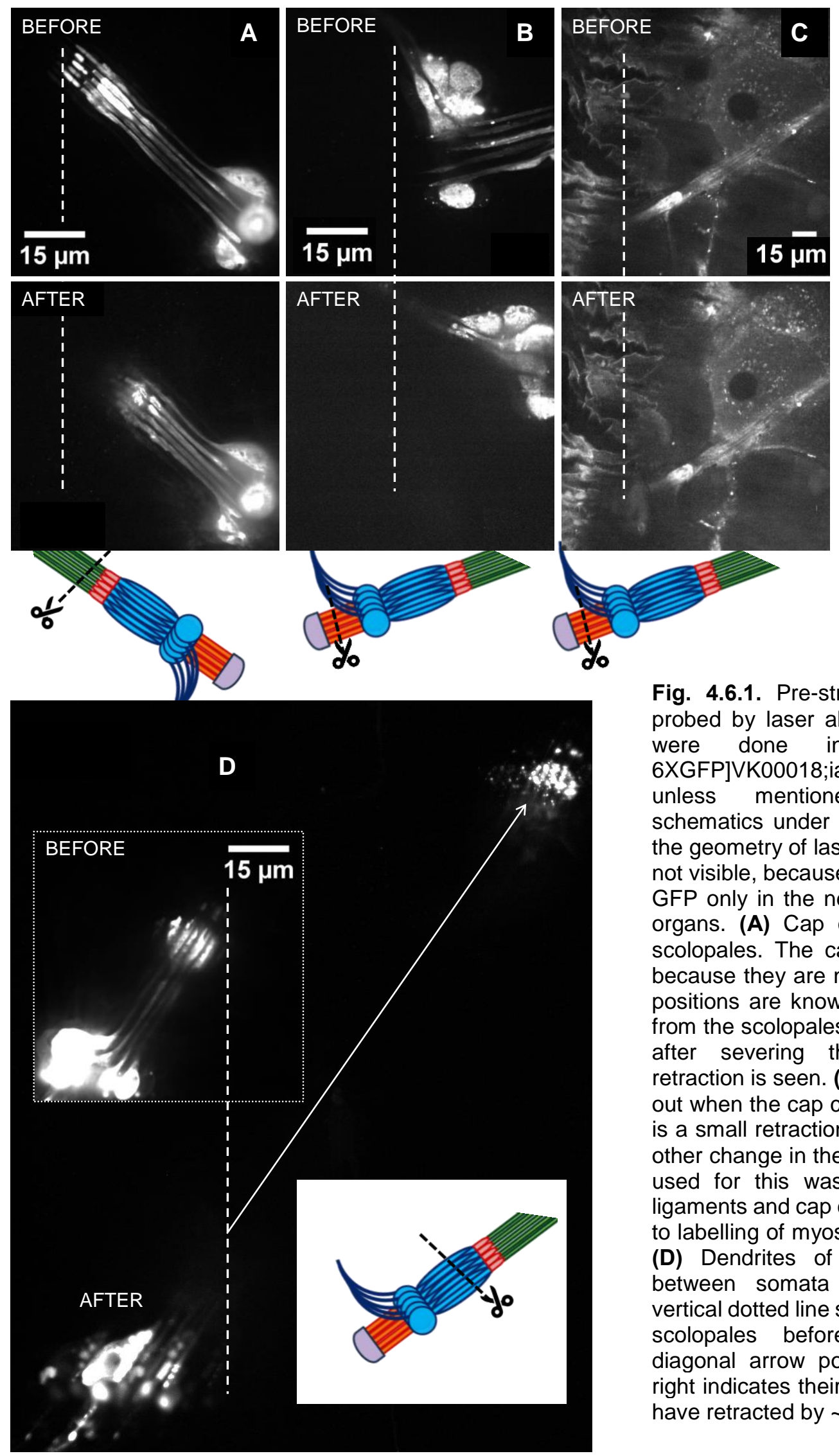

Fig. 4.6.1. Pre-strain in the Ich5 organ probed by laser ablation. All experiments were done in w[-];PBac[20XUAS6XGFP]VK00018;iav-GAL4,w[+]/+ larvae unless mentioned otherwise. The schematics under the sub-figures indicate the geometry of laser cutting. Cap cells are not visible, because the animals expressed GFP only in the neuronal part of the Ich5 organs. (A) Cap cells severed near the scolopales. The cap cells are not visible because they are not fluorescent, but their positions are known because they extend from the scolopales. (B) Ligament cells cut after severing the cap cells. Some retraction is seen. (C) If the same is carried out when the cap cells are still intact, there is a small retraction, less than in $\mathbf{B}$, but no other change in the lch5 organ. The fly line used for this was Sqh-GFP, where the ligaments and cap cells are fluorescent due to labelling of myosin, but not the neurons. (D) Dendrites of the neurons severed between somata and scolopales. The vertical dotted line shows the position of the scolopales before ablation, and the diagonal arrow pointing towards the top right indicates their new position after they have retracted by $\sim 100 \mu \mathrm{m}$. 


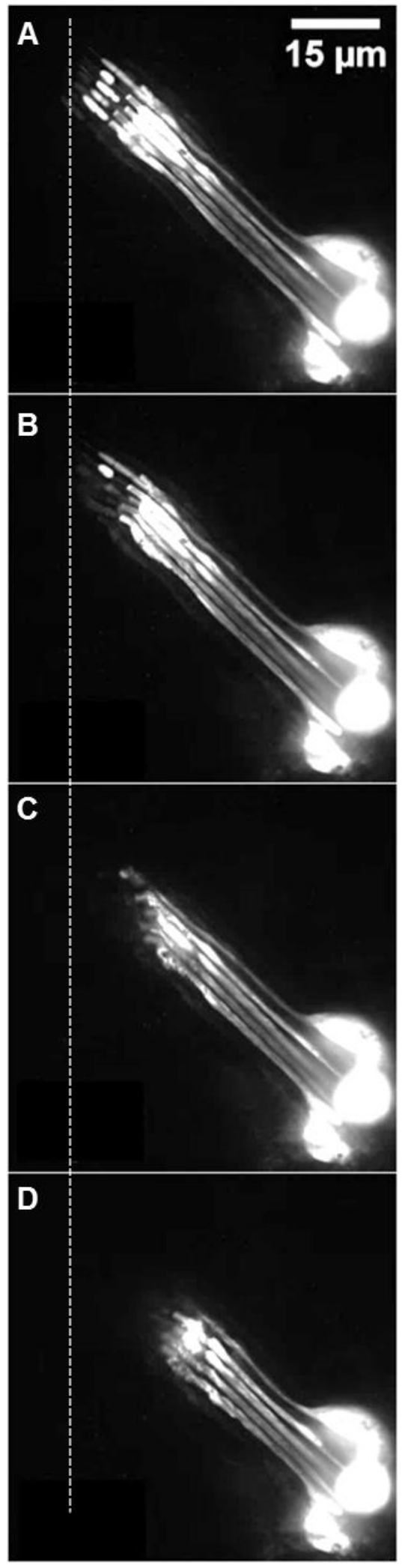

Fig. 4.6.2. Relaxation of Ich5 organ during sequential severing of cap cells by laser ablation near the scolopales (cf. Fig. 4.6.1 A). Only the neurons express GFP. (A) Before ablation. Panels (B) to (D) show retraction of the neuronal dendrites as the cap cells are severed one by one. (B) After severing one cap cell from the bottom. (C) After severing three cap cells from the bottom (D) after severing all five cap cells. 


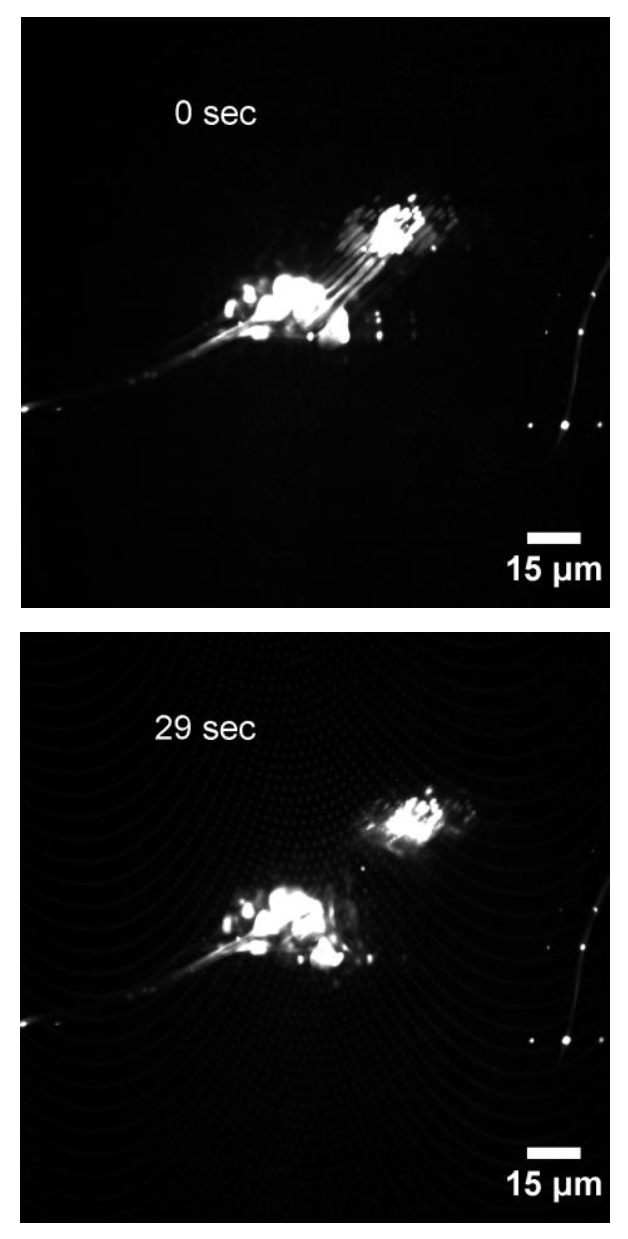

$24 \mathrm{sec}$
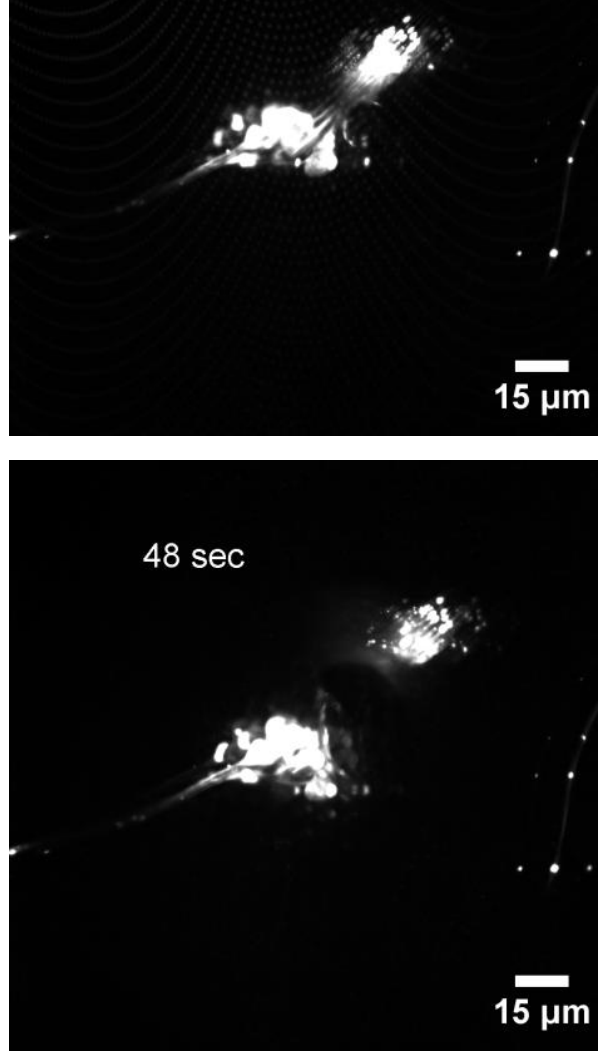

$96 \mathrm{sec}$
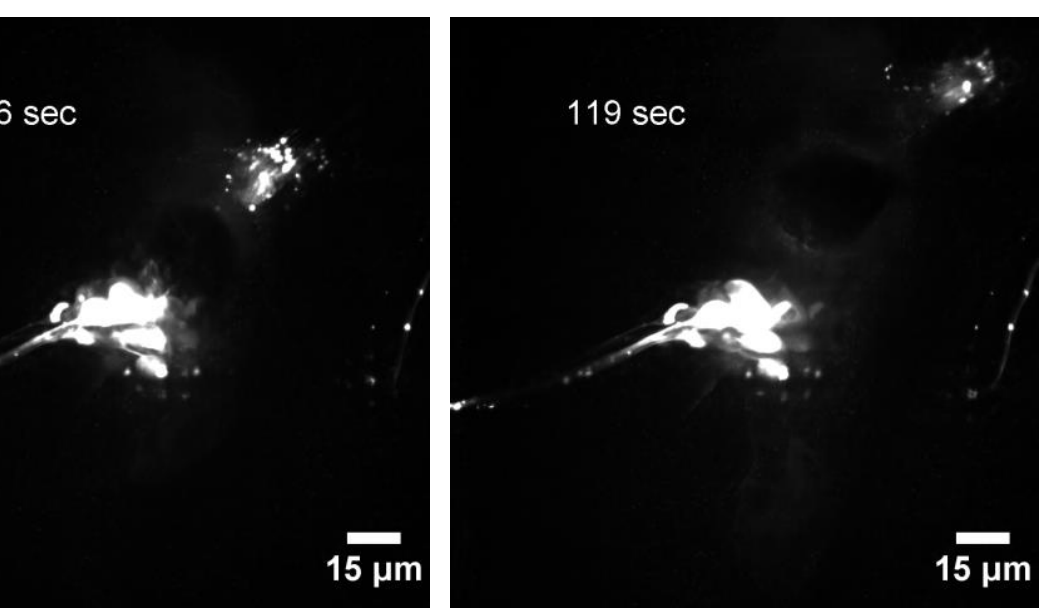

$131 \mathrm{sec}$

$127 \mathrm{sec}$

$15 \mu \mathrm{m}$

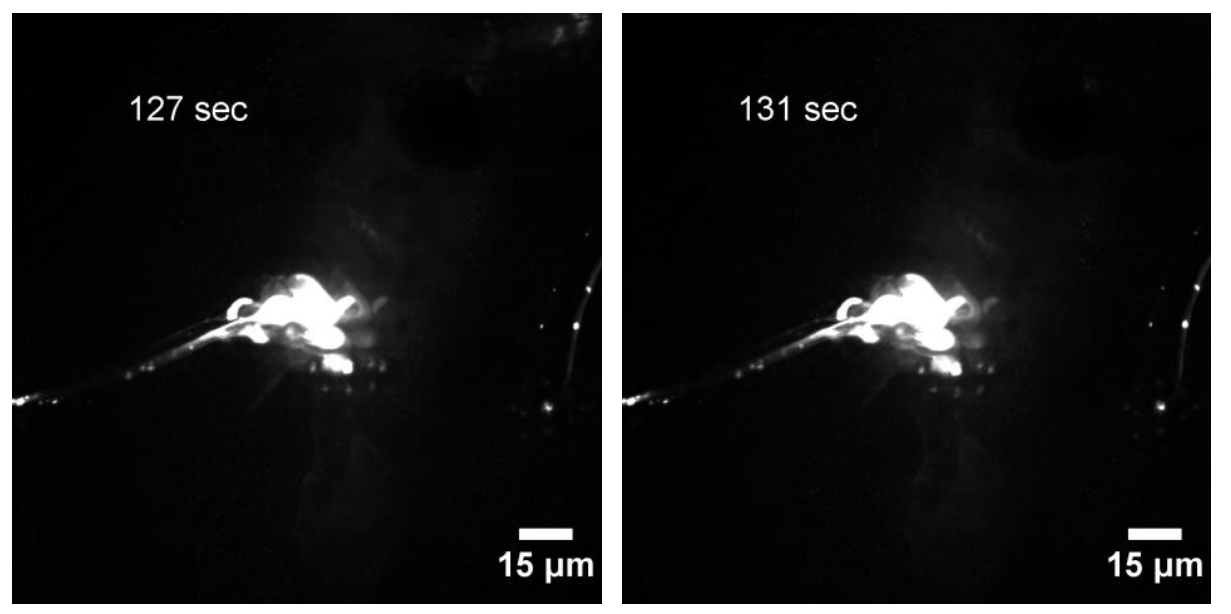

Fig. 4.6.3. Time course of laser ablation. Here, the dendrites of the Ich5 organ in a w[-];PBac[20XUAS6XGFPJVK00018;iav-

$\mathrm{GAL} 4, \mathrm{w}[+] /+$ larva were severed one by one. The ablation began at $20 \mathrm{sec}$, and by $120 \mathrm{sec}$ the rapid retraction had already begun. 

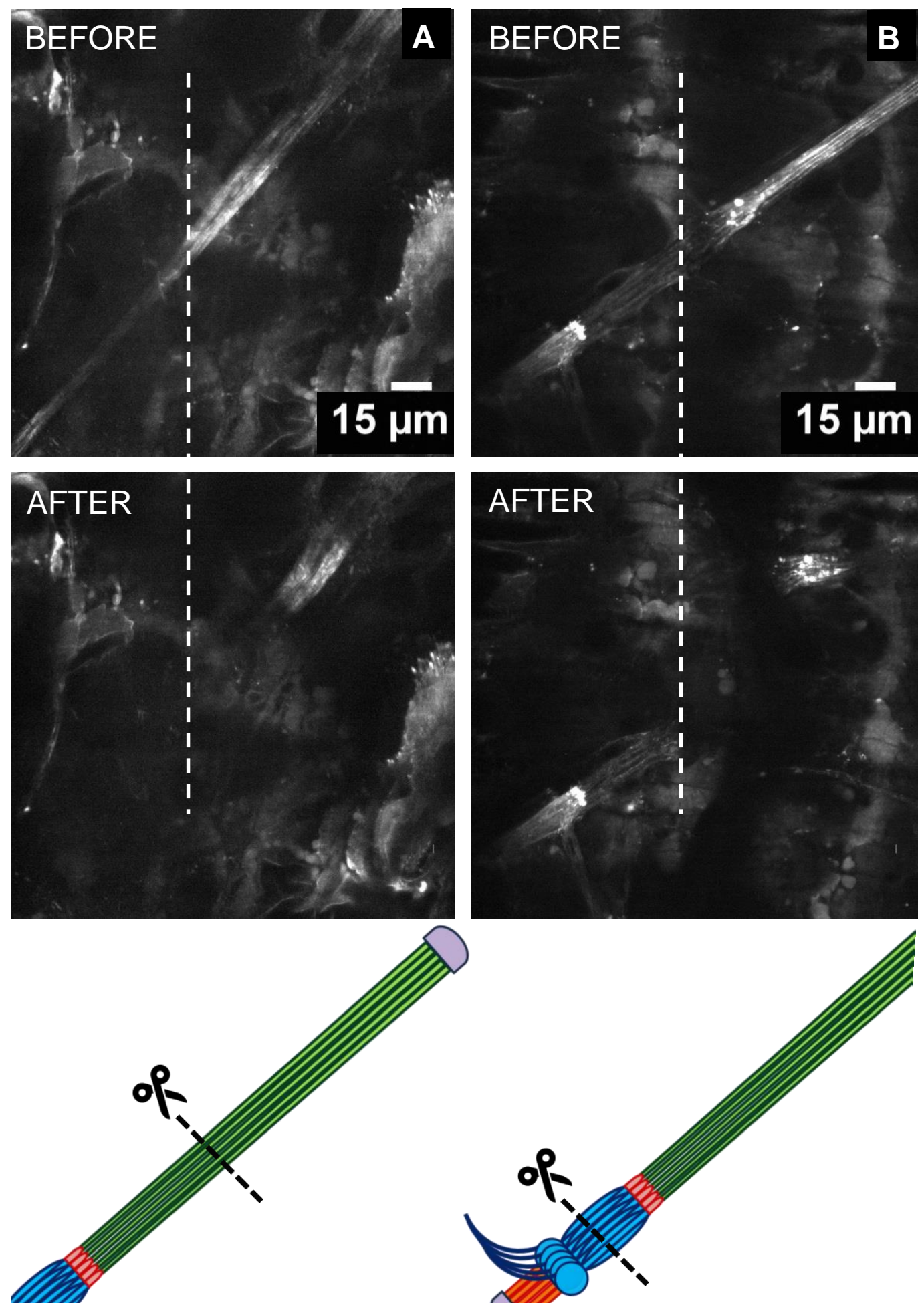

Fig. 4.6.4. Relaxation of Ich5 organ in Sqh-GFP larvae during laser ablation. GFP is expressed in ligaments and cap cells. (A) After severing the cap cells alone (B) After severing the dendrites. The retraction in (A) is comparable to that in Fig. 4.6.1 D, while in $(\mathrm{B})$ it is slightly less. 

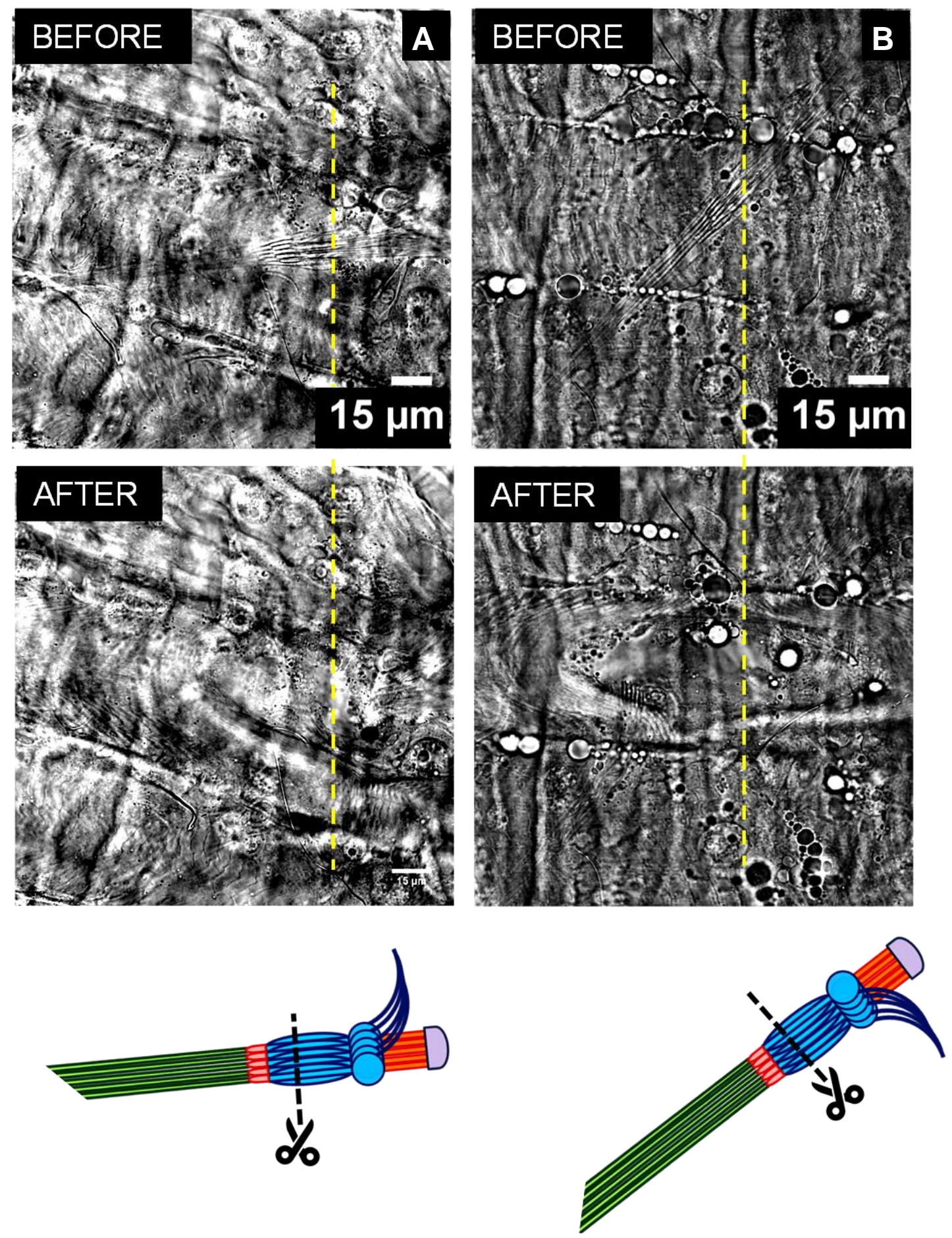

Fig. 4.6.5. (A) Relaxation of Ich5 organ in the control: w118 X Pinta GAL4, after severing the dendrites. Here the retraction is so strong that the cap cells leave the image plane. (B) Relaxation of Ich5 organ in the myosin knockdown mutant, after severing the dendrites. There is no fluorescence in these two cases. The retraction in $(B)$ is much less than that in $(A)$. 


\subsection{Measurement of forces using calibrated glass pipettes}

For these experiments, we used fillet preparations with muscles excised (section 3.8.4). We used the set-up as described in section 3.10 and Figs. 3.10.1-3.10.2. The glass pipette was placed with its short arm parallel to the lch5 organ such that its downward-pointing tip was in contact with the organ. The stage was moved so that the lch5 organ pushed against the tip of the pipette. We expected to observe a deflection in the pipette when the lch5 organ disengaged from it, but the pipette was seen to be pulled along with the organ. Another problem encountered was that even when the muscles are excised, there is still a lot of debris in the sample, to which the pipette tends to adhere.

We were recently able to obtain a preparation with negligible debris. We repeated the experiment, this time moving not the stage but the pipette by known distances, by controlling the micromanipulator with the inbuilt computer software. This is an improvement in the technique, and points to future experiments that will yield more quantitative results. Fig. 4.7.1 shows the Ich5 organ as it is displaced by the glass pipette.
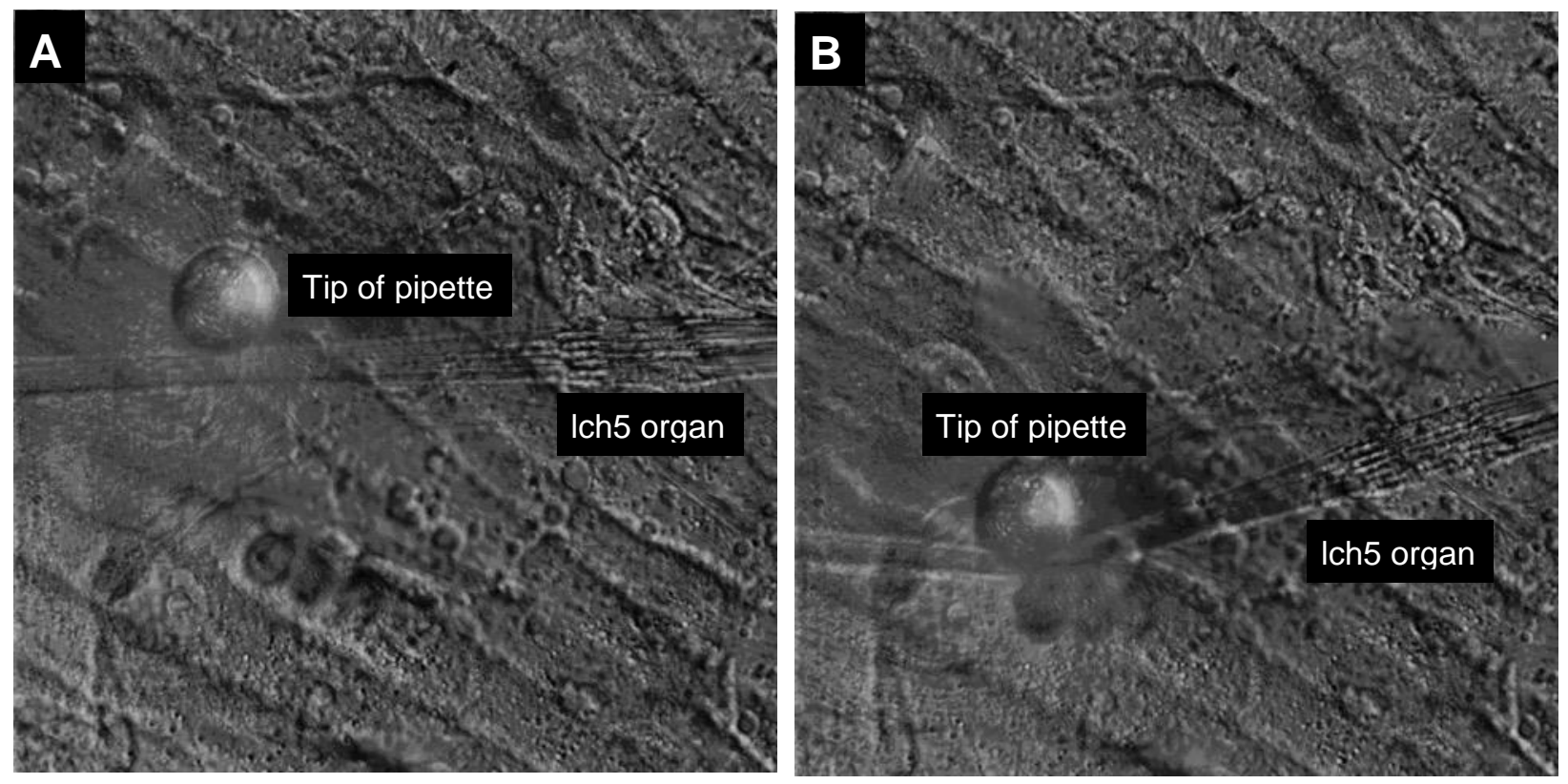

Fig. 4.7.1. (A) Ich5 organ in the relaxed configuration (B) Displaced by $100 \mu \mathrm{m}$ using the computer-controlled glass pipette 


\section{Conclusions \& Outlook}

During my PhD thesis, I worked on several experiments focused on the mechanics of the Ich5 organ in the Drosophila larva. The results presented in this thesis led to three main conclusions.

First, we found that the lch5 organs of Drosophila larvae show remarkable stretch elasticity with a large linear range (Fig. 4.5.1, and Fig. 4.6.1 D). When stretched and released, they relax in an overdamped fashion, viscously, but not elastically coupled to overlying muscle layers (Figs. 4.4.4 4.4.6). Upon laser cutting, the organ recoils with a large amplitude, which demonstrates a large pre-strain of $\sim 50 \%$ in the cap cells of the functioning organ. In the future, it will be interesting to explore the role of this pre-strain and the associated pre-tension and its regulation in the sensing mechanism of Ich5 chordotonal organs. It is tempting to hypothesize that this tension is actively regulated and that it facilitates mechanotransduction in chordotonal sensory neurons, e.g. by maximizing the sensitivity of mechanotransduction channels through keeping their open probability at rest at its half maximum value, where small mechanical stimuli cause the maximal open probability change.

Second, we show that non-muscle myosin II is the main molecular motor involved in initial proprioceptory signal detection in the chordotonal organs of the Drosophila larva. Given the abundance of non-muscle myosin II in the cap cells, we hypothesized that the greater increase in the length of the cap cells as compared to the neurons in our shape dynamics experiments (section 4.5 and Fig. 4.5.2), as well as their strong retraction in the laser ablation experiments, must be related to the presence of non-muscle myosin II. Indeed, when we repeated the ablation experiment for a myosin knockdown mutant Sqh RNAi X Pinta GAL4, we observed that the cap cells retracted by a much lower amount than for the control $w^{118} X$ Pinta GAL4 (Fig. 4.6.5). In addition, the transgenic line Sqh-GFP, where the spaghetti squash light chains of myosin II are labeled with GFP, showed a slightly lower retraction than the control - 80 $\mu \mathrm{m}$ as compared to $\sim 100 \mu \mathrm{m}$ (Fig. 4.6.4). This could be due to a possible reduction in myosin activity induced by the labelling. It would be interesting to compare this with similar studies in other insects, and possibly mechanosensors of other animal taxa as well, such as vertebrates.

Third, we have successfully devised a method to probe the Ich5 organ using a glass pipette, as described in section 4.7. However, these experiments should still be considered preliminary, and indicate a potential for future investigations. It would be intriguing to investigate whether there is indeed a difference in the forces measured from the wild type and from the myosin knockdown 
mutant. We expect that later investigations in this direction will lead us to a better understanding of the magnitude and nature of the forces at play in the functioning of the Ich5 organ.

Another area that is yet unexplored is the impact of myosin-inhibitory drugs on chordotonal organs. Blebbistatin is a commonly used myosin inhibitory drug $(174,175)$, but Drosophila non-muscle myosin II is insensitive to it $(116,176)$. The drug Y-27632, an inhibitor of the kinase activity of ROCK-I and ROCK-II, both of which phosphorylate non-muscle myosin II (177), appears to be a better candidate for this. This drug has already been successfully used in the Drosophila embryo to inhibit various mechanical aspects of development (121,178-181), but to the best of our knowledge, it has not yet been applied to larvae. A suitable method to inject the drug into the lch5 organ needs to be developed. It would indeed be interesting to repeat our experiments after applying this drug, and observe how the response of the organ differs from the case where the drug has not been applied. 


\section{Appendix A1 \\ Calculation of increase in length of Ich5 organ in the fillet preparation}

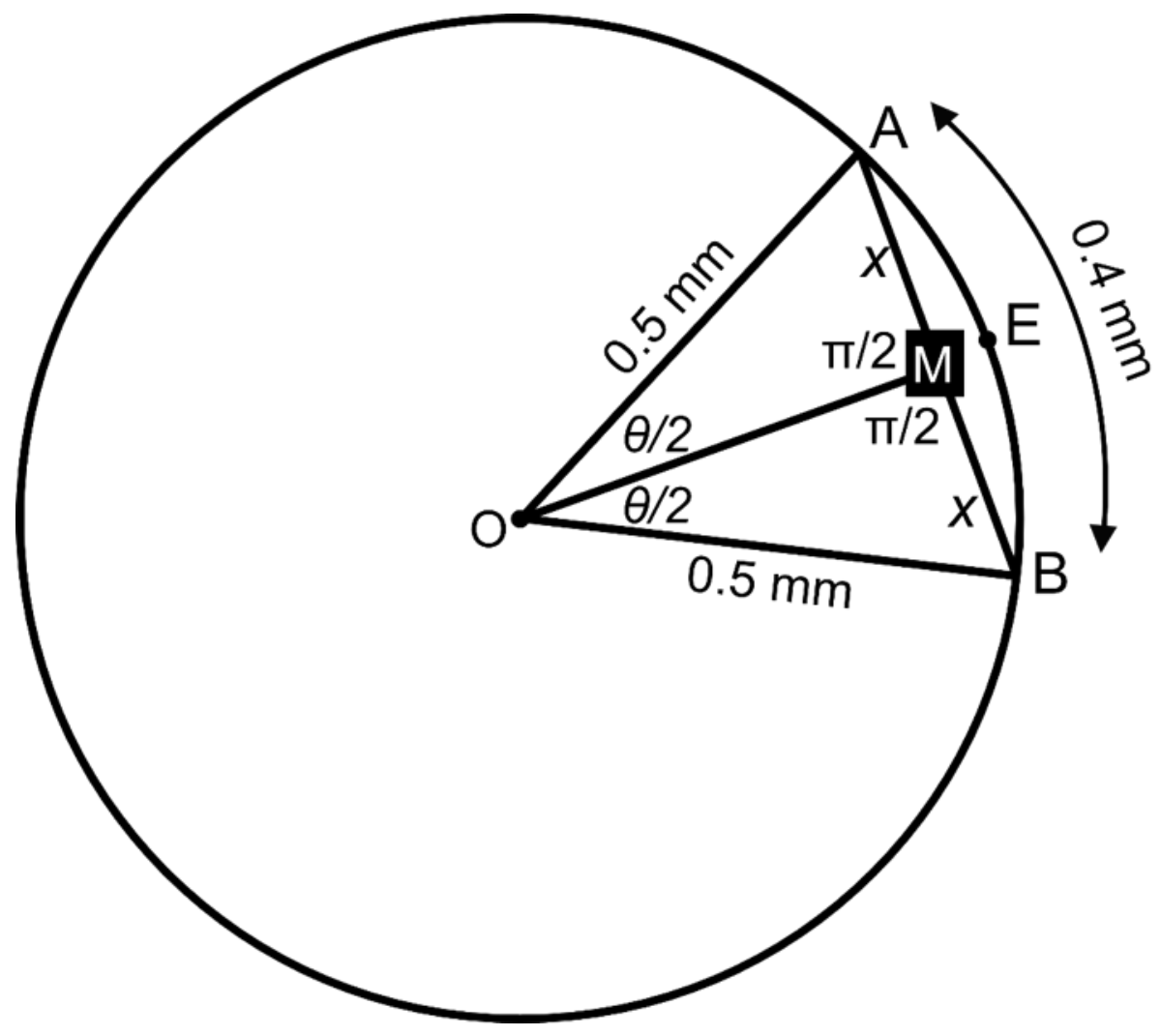

Fig. A1.1. Geometric visualization of a cross-section of a third instar Drosophila larva. Here AB represents the Ich5 organ.

The thickness of a Drosophila larva in the third instar stage is of the order of $1 \mathrm{~mm}$. Let us then consider the larva as a cylinder of diameter $1 \mathrm{~mm}$. Let us take a circular cross-section of this cylinder, along a plane perpendicular to the body axis and containing an lch5 organ. The organ is then a chord of the circle (Fig. A1.1). For convenience, we shall call the centre of the circle $O$, and the attachment points of the Ich5 organ $A$ and $B$.

In the fillet prep, the larva is flattened out. The arc AEB of the circle that contains the chord would then become a straight line segment, and the chord AB, i.e. the lch5 organ, would get stretched. Its new length then equals the length of the arc. We treat this length as being equal to $0.4 \mathrm{~mm}$ (400 $\mu \mathrm{m})$, since that is the typical length of an Ich5 organ in a larval fillet preparation.

Radius $\rho=0.5 \mathrm{~mm}=\mathrm{OA}=\mathrm{OB}$

Arc length $L=0.4 \mathrm{~mm}$ 
Angle subtended by the arc, in radians $=\theta=N / L$

$=0.4 / 0.5=0.8$

Let the midpoint of the chord $A B$ be $M$. Then the line segment $O M$ is perpendicular to $A B$. $A M=M B$ $=x$. Then the original length of the Ich5 organ, $\lambda$, is $2 x$.

AM divides the angle $\theta$ into two angles each of magnitude $\theta / 2=0.4 \mathrm{rad}$. This means

$\sin \theta / 2=\mathrm{AM} / \mathrm{OA}=x / 0.5$

Then $x=0.5 \sin \theta / 2=0.5 \sin 0.4$

$\lambda=\sin 0.4 \approx 389.4 \mu \mathrm{m}$

Increase in length $=(1-N L) * 100$

$\approx 2.6 \%$ 


\section{Appendix A2 \\ Text and figures from the submitted manuscript}

The following text and figures in this thesis have been used mostly verbatim from the recently submitted manuscript "Mechanical properties of a Drosophila larval chordotonal organ", with slight modification in some cases. The numbering of sections and figures is different from the paper.

1. Figs. 2.4.2 and 2.4.3, and the accompanying captions

2. Sections 3.8.1 - 3.8.3 (entire sections)

3. Section 3.8.4:

"We used third instar Canton-S ... larva thus prepared is known as a fillet"

4. Section 3.8.5:

i. "The fillet preparation was viewed using ... camera (Photron Fastcam, VKT Video Kommunikation $\mathrm{GmbH}$, Pfullingen, Germany)."

ii. "using a micromanipulator (Scientifica UI-1000-i, Multichannel Systems, Reutlingen, Germany), the tungsten needle was brought into focus ... released."

5. Section 3.9:

"A spinning disc confocal microscope (CSU-X1, Carl Zeiss)... AxioCam MRm camera (Carl Zeiss) at a rate of $1 \mathrm{fps."}$

6. Section 4.4:

i. "We observed the relaxation dynamics of the deformed organ ... fast snap-back and a slow relaxation"

ii. "In fillet preparations with the muscle layers above the organ ... well-defined resonance frequency."

iii. "Most biological materials ... bared lch5 organs."

iv. Figures $4.4 .4-4.4 .6$ and the accompanying captions

v. Tables 4.4.1 - 4.4.2 and the accompanying captions

7. Section 4.5 (entire section)

8. Section 4.6:

i. "The straight conformation of the Ich5 organ ... regulate - organ tension."

ii. $\quad$ Fig. 4.6.1 (except part C), Fig. 4.6.2, Fig. 4.6.3 
9. Section 5 (Conclusions and Outlook):

"We found that the Ich5 organs of Drosophila larvae ... maximal open probability change." 


\section{References}

1. French, A. S. 1992. Mechanotransduction. Annu Rev Physiol 54:135-152.

2. Wirtz, D., K. Konstantopoulos, and P. C. Searson. 2011. The physics of cancer: the role of physical interactions and mechanical forces in metastasis. Nat Rev Cancer 11:512-522.

3. Tedeschi, F., Carbone, G. 2014. Design Issues for Hexapod Walking Robots. Robotics 3.

4. McDermott, H. 1998. A programmable sound processor for advanced hearing aid research. IEEE Transactions on Rehabilitation Engineering 6:53-59.

5. Martin, P., and A. J. Hudspeth. 1999. Active hair-bundle movements can amplify a hair cell's response to oscillatory mechanical stimuli. P Natl Acad Sci USA 96:14306-14311.

6. Perozo, E., D. M. Cortes, P. Sompornpisut, A. Kloda, and B. Martinac. 2002. Open channel structure of MscL and the gating mechanism of mechanosensitive channels. Nature 418:942948.

7. Hardie, R. C., and P. Raghu. 2001. Visual transduction in Drosophila. Nature 413:186-193.

8. Hubbell, W. L., and M. D. Bownds. 1979. Visual Transduction in Vertebrate Photoreceptors. Annu Rev Neurosci 2:17-34.

9. Montell, C. 1999. Visual transduction in Drosophila. Annu Rev Cell Dev Bi 15:231-268.

10. Pepe, I. M., I. Panfoli, and H. E. Hamm. 1989. Visual Transduction in Vertebrate Photoreceptors - Light Activation of Guanylate-Cyclase. Cell Biophys 14:129-137.

11. Ache, B. W., and A. Zhainazarov. 1995. Dual Second-Messenger Pathways in Olfactory Transduction. Curr Opin Neurobiol 5:461-466.

12. Albert, J. T., B. Nadrowski, and M. C. Göpfert. 2007. Mechanical signatures of transducer gating in the Drosophila ear. Curr Biol 17:1000-1006.

13. Chiquet, M., Tunç-Civelek, V., Sarasa-Renedo, A. 2007. Gene regulation by mechanotransduction in fibroblasts. Applied Physiology, Nutrition, and Metabolism 32:967973.

14. Chang, S., R. J. Johnston, C. Frokjaer-Jensen, S. Lockery, and O. Hobert. 2004. MicroRNAs act sequentially and asymmetrically to control chemosensory laterality in the nematode. Nature 430:785-789.

15. Herness, M. S., and T. A. Gilbertson. 1999. Cellular mechanisms of taste transduction. Annu Rev Physiol 61:873-900.

16. Arnadottir, J., and M. Chalfie. 2010. Eukaryotic Mechanosensitive Channels. Annu Rev Biophys 39:111-137.

17. Christensen, A. P., and D. P. Corey. 2007. TRP channels in mechanosensation: direct or indirect activation? Nat Rev Neurosci 8:510-521.

18. Yan, Z. Q., W. Zhang, Y. He, D. Gorczyca, Y. Xiang, L. E. Cheng, S. Meltzer, L. Y. Jan, and Y. N. Jan. 2013. Drosophila NOMPC is a mechanotransduction channel subunit for gentletouch sensation. Nature 493:221-225.

19. Hudspeth, A. J. 2008. Making an effort to listen: Mechanical amplification in the ear. Neuron 59:530-545.

20. Martin, P., and A. J. Hudspeth. 2001. Compressive nonlinearity in the hair bundle's active response to mechanical stimulation. $P$ Natl Acad Sci USA 98:14386-14391.

21. Martin, P., A. J. Hudspeth, and F. Julicher. 2001. Comparison of a hair bundle's spontaneous oscillations with its response to mechanical stimulation reveals the underlying active process. P Natl Acad Sci USA 98:14380-14385.

22. Andres, M., S. Karak, S. H. Joo, D. Piepenbrock, and M. Gopfert. 2013. Drosophila Melanogaster as a Model to Understand Hearing in Disease Vectors. Pathog Glob Health 107:406-406.

23. Gopfert, M. C., and R. M. Hennig. 2016. Hearing in Insects. Annu Rev Entomol 61:257-276.

24. Kamikouchi, A., H. K. Inagaki, T. Effertz, O. Hendrich, A. Fiala, M. C. Göpfert, and K. Ito. 2009. The neural basis of Drosophila gravity-sensing and hearing. Nature 458:165-U161.

25. Nadrowski, B., J. T. Albert, and M. C. Göpfert. 2008. Transducer-based force generation explains active process in Drosophila hearing. Curr Biol 18:1365-1372. 
26. Arshavsky, V. Y., Burns, M. E. . 2014. Current understanding of signal amplification in phototransduction. Cellular Logistics 4:e29390.

27. Battle, C. G. 2013. Mechanics \& Dynamics of the Primary Cilium (PhD thesis). In Third Institute of Physics - Biophysics, Faculty of Physics. University of Göttingen, Germany.

28. Paluch, E. K., C. M. Nelson, N. Biais, B. Fabry, J. Moeller, B. L. Pruitt, C. Wollnik, G. Kudryasheva, F. Rehfeldt, and W. Federle. 2015. Mechanotransduction: use the force(s). Bmc Biol 13.

29. Grima, R. 2007. Directed cell migration in the presence of obstacles. Theor Biol Med Model 4.

30. de Langre, E. 2008. Effects of Wind on Plants. Annual Review of Fluid Mechanics 40:141168

31. Braam, J. 2005. In touch: plant responses to mechanical stimuli. New Phytol 165:373-389.

32. Sack, F. D. 1991. Plant Gravity Sensing. Int Rev Cytol 127:193-252.

33. Coutand, C., M. Chevolot, A. Lacointe, N. Rowe, and I. Scotti. 2010. Mechanosensing of stem bending and its interspecific variability in five neotropical rainforest species. Ann BotLondon 105:341-347.

34. Kingwell, K. 2010. Proprioception: Sensational mechanics. Nat Rev Neurosci 11:665.

35. Caldwell, J. C., M. M. Miller, S. Wing, D. R. Soll, and D. F. Eberl. 2003. Dynamic analysis of larval locomotion in Drosophila chordotonal organ mutants. Proc Natl Acad Sci U S A 100:16053-16058.

36. Dietz, V. 2002. Proprioception and locomotor disorders. Nat Rev Neurosci 3:781-790.

37. Aleman-Meza, B., S. K. Jung, and W. W. Zhong. 2015. An automated system for quantitative analysis of Drosophila larval locomotion. Bmc Developmental Biology 15.

38. Berni, J. 2015. Genetic Dissection of a Regionally Differentiated Network for Exploratory Behavior in Drosophila Larvae. Curr Biol 25:1319-1326.

39. Berrigan, D., and D. J. Pepin. 1995. How Maggots Move - Allometry and Kinematics of Crawling in Larval Diptera. J Insect Physiol 41:329-337.

40. Gjorgjieva, J., J. Berni, J. F. Evers, and S. J. Eglen. 2013. Neural circuits for peristaltic wave propagation in crawling Drosophila larvae: analysis and modeling. Front Comput Neurosc 7.

41. Halachmi, N., A. Nachman, and A. Salzberg. 2012. Visualization of proprioceptors in Drosophila larvae and pupae. J Vis Exp:e3846.

42. Heckscher, E. S., S. R. Lockery, and C. Q. Doe. 2012. Characterization of Drosophila Larval Crawling at the Level of Organism, Segment, and Somatic Body Wall Musculature. J Neurosci 32:12460-12471.

43. Marley, R., R. A. Baines. 2011a. Dissection of first- and second-instar Drosophila larvae for electrophysiological recording from neurons: The flat (or fillet) preparation. Cold Spring Harbor Protocols.

44. Zhang, W., Z. Yan, L. Y. Jan, and Y. N. Jan. 2013. Sound response mediated by the TRP channels NOMPC, NANCHUNG, and INACTIVE in chordotonal organs of Drosophila larvae. Proc Natl Acad Sci U S A 110:13612-13617.

45. Grueber, W. B., B. Ye, C. H. Yang, S. Younger, K. Borden, L. Y. Jan, and Y. N. Jan. 2007. Projections of Drosophila multidendritic neurons in the central nervous system: links with peripheral dendrite morphology. Development 134:55-64.

46. Kernan, M. J. 2007. Mechanotransduction and auditory transduction in Drosophila. Pflugers Archiv-European Journal of Physiology 454:703-720.

47. Berlese, A. 1909. Gli insetti: Embriologia e morfologia. Società Editrice libraria, Milan.

48. Mciver, S. B. 1975. Structure of Cuticular Mechanoreceptors of Arthropods. Annu Rev Entomol 20:381-397.

49. Hertweck, H. 1931. Anatomie und Variabilität des Nervensystems und der Sinnesorgane von Drosophila melanogaster (Meigen) (PhD thesis) University of Frankfurt, Akademische Verlagsgesellschaft m. b. H. Leipzig.

50. Field, L. H., and T. Matheson. 1998. Chordotonal organs of insects. Advances in Insect Physiology, Vol 27 27:1-228.

51. Gillespie, P. G., and R. G. Walker. 2001. Molecular basis of mechanosensory transduction. Nature 413:194-202.

52. Yack, J. E. 2004. The structure and function of auditory chordotonal organs in insects. Microsc Res Techniq 63:315-337. 
53. Altman, J. S., and N. M. Tyrer. 1977. The Locust Wing Hinge Stretch Receptors . 1. Primary Sensory Neurons with Enormous Central Arborizations. J Comp Neurol 172:409-430.

54. Simon, M. A., and B. A. Trimmer. 2009. Movement encoding by a stretch receptor in the softbodied caterpillar, Manduca sexta. J Exp Biol 212:1021-1031.

55. Yack, J. E., and J. H. Fullard. 1993. Proprioceptive Activity of the Wing-Hinge StretchReceptor in Manduca-Sexta and Other Atympanate Moths - a Study of the Noctuoid Moth Ear B-Cell Homolog. J Comp Physiol A 173:301-307.

56. Theophilidis, G., and M. D. Burns. 1979. A Muscle Tension Receptor in the Locust Leg. J Comp Physiol 131:247-254.

57. Gelperin, A. 1967. Stretch Receptors in the Foregut of the Blowfly. Science 157:208-\&.

58. Tracey, W. D., R. I. Wilson, G. Laurent, and S. Benzer. 2003. painless, a Drosophila gene essential for nociception. Cell 113:261-273.

59. Ainsley, J. A., J. M. Pettus, D. Bosenko, C. E. Gerstein, N. Zinkevich, M. G. Anderson, C. M. Adams, M. J. Welsh, and W. A. Johnson. 2003. Enhanced locomotion caused by loss of the Drosophila DEG/ENaC protein pickpocket1. Curr Biol 13:1557-1563.

60. Chevalier, R. L. 1969. The Fine Structure of Campaniform Sensilla on the Halteres of Drosophila Melanogaster. J Morphol 128:443-+.

61. Moran, D. T., K. M. Chapman, and R. A. Ellis. 1971. The Fine Structure of Cockroach Campaniform-Sensilla. J Cell Biol 48:155-\&.

62. Gnatzy, W., U. Grunert, and M. Bender. 1987. Campaniform Sensilla of Calliphora-Vicina (Insecta, Diptera) .1. Topography. Zoomorphology 106:312-319.

63. Newland, P. L., and N. J. Emptage. 1996. The central connections and actions during walking of tibial campaniform sensilla in the locust. J Comp Physiol A 178:749-762.

64. Lees, A. D. 1942. Homology of the Campaniform Organs on the Wing of Drosophila melanogaster. Nature 150.

65. Chapman, K. M., J. L. Mosinger, R. B. Duckrow. 1979. The role of distributed viscoelastic coupling in sensory adaptation in an insect mechanoreceptor. J Comp Physiol A Neuroethol Sens Neural Behav Physiol 131:1-12.

66. http://www.biology-resources.com/insect-structure.html.

67. Furman, D. P., and T. A. Bukharina. 2008. How Drosophila melanogaster forms its mechanoreceptors. Curr Genomics 9:312-323.

68. Graber, V. 1881 Über die stifteführenden oder chordotonalen Sinnesorgane bei der Insecte. Zoologischer Anzeiger 4: 450-453.

69. Halachmi, N., A. Nachman, and A. Salzberg. 2012. Visualization of Proprioceptors in Drosophila Larvae and Pupae. Jove-Journal of Visualized Experiments.

70. Moran, D. T., F. J. Varela, and J. C. Rowley, 3rd. 1977. Evidence for active role of cilia in sensory transduction. Proc Natl Acad Sci U S A 74:793-797.

71. Halachmi, N., A. Nachman, and A. Salzberg. 2016. A newly identified type of attachment cell is critical for normal patterning of chordotonal neurons. Developmental Biology 411:61-71.

72. Klein, Y., N. Halachmi, N. Egoz-Matia, M. Toder, and A. Salzberg. 2010. The proprioceptive and contractile systems in Drosophila are both patterned by the EGR family transcription factor Stripe. Developmental Biology 337:458-470.

73. Caldwell, J. C., M. M. Miller, S. Wing, D. R. Soll, and D. F. Eberl. 2003. Dynamic analysis of larval locomotion in Drosophila chordotonal organ mutants. P Natl Acad Sci USA 100:1605316058.

74. Wheeler, W. M. 1965. Ants: Their Structure, Development and Behavior. Columbia University Press, New York.

75. Graber, V. 1881. Die chordotonalen Sinnesorgane und das Gehör der Insecten. Archiv für mikroskopische Anatomie 20:506-640.

76. von Siebold, C. T. 1844. Über das Stimm-und Gehörorgan der Orthopteren. Archiv für Naturgeschichte 10:52-81.

77. Göpfert, M. C., and D. Robert. 2002. The mechanical basis of Drosophila audition. J Exp Biol 205:1199-1208.

78. Karak, S., J. S. Jacobs, M. Kittelmann, C. Spalthoff, R. Katana, E. Sivan-Loukianova, M. A. Schon, M. J. Kernan, D. F. Eberl, and M. C. Gopfert. 2015. Diverse Roles of Axonemal Dyneins in Drosophila Auditory Neuron Function and Mechanical Amplification in Hearing. Sci Rep-Uk 5. 
79. Yack, J. E. 2004. The structure and function of auditory chordotonal organs in insects. Microsc Res Tech 63:315-337.

80. Lee, J., S. Moon, Y. Cha, and Y. D. Chung. 2010. Drosophila TRPN(=NOMPC) channel localizes to the distal end of mechanosensory cilia. Plos One 5:e11012.

81. Karak, S. 2013. Axonemal dyneins and force generation by neurons in Drosophila melanogaster ear (PhD thesis) In Department of Cellular Neurobiology, Schwann-Schleiden Research Centre. University of Göttingen, Germany.

82. Senthilan, P. R., D. Piepenbrock, G. Ovezmyradov, B. Nadrowski, S. Bechstedt, S. Pauls, M. Winkler, W. Mobius, J. Howard, and M. C. Göpfert. 2012. Drosophila Auditory Organ Genes and Genetic Hearing Defects. Cell 150:1042-1054.

83. DiCaprio, R. A., H. Wolf, and A. Büschges. 2002. Activity-dependent sensitivity of proprioceptive sensory neurons in the stick insect femoral chordotonal organ. Journal of Neurophysiology 88:2387-2398.

84. Rydqvist, B., N. Purali, and J. Lannergren. 1994. Viscoelastic Properties of the Rapidly Adapting Stretch-Receptor Muscle of the Crayfish. Acta Physiologica Scandinavica 150:151159.

85. Jennings, B. H. 2011. Drosophila - a versatile model in biology \& medicine. Mater Today 14:190-195.

86. Cole, E. S., and J. Palka. 1982. The Pattern of Campaniform Sensilla on the Wing and Haltere of Drosophila-Melanogaster and Several of Its Homeotic Mutants. J Embryol Exp Morph 71:41-61.

87. Shimono, K., A. Fujimoto, T. Tsuyama, M. Yamamoto-Kochi, M. Sato, Y. Hattori, K. Sugimura, T. Usui, K. Kimura, and T. Uemura. 2009. Multidendritic sensory neurons in the adult Drosophila abdomen: origins, dendritic morphology, and segment- and age-dependent programmed cell death. Neural Dev 4.

88. http://flypress.gen.cam.ac.uk/?page $\mathrm{id}=21$.

89. Yack, J. E., and J. H. Fullard. 1993. What Is an Insect Ear. Ann Entomol Soc Am 86:677682.

90. Hedwig, B. 1988. Activation and Modulation of Auditory Receptors in Locusta-Migratoria by Respiratory Movements. J Comp Physiol A 162:237-246.

91. van Staaden, M. J., M. Rieser, S. R. Ott, M. A. Pabst, and H. Romer. 2003. Serial hearing organs in the atympanate grasshopper Bullacris membracioides (Orthoptera, Pneumoridae). J Comp Neurol 465:579-592.

92. Andres, M., M. Seifert, C. Spalthoff, B. Warren, L. Weiss, D. Giraldo, M. Winkler, S. Pauls, and M. C. Gopfert. 2016. Auditory Efferent System Modulates Mosquito Hearing. Curr Biol 26:2028-2036.

93. Gibson, G., B. Warren, and I. J. Russell. 2010. Humming in Tune: Sex and Species Recognition by Mosquitoes on the Wing. Jaro-J Assoc Res Oto 11:527-540.

94. Ai, H., J. Rybak, R. Menzel, and T. Itoh. 2009. Response Characteristics of VibrationSensitive Interneurons Related to Johnston's Organ in the Honeybee, Apis mellifera. J Comp Neurol 515:145-160.

95. Inagaki, H., A. Kamikouchi, T. Effertz, A. Fiala, M. C. Gopfert, and K. Ito. 2009. The neural basis of Drosophila gravity sensing and hearing. J Neurogenet 23:S69-S70.

96. Sun, Y. S., L. Liu, Y. Ben-Shahar, J. S. Jacobs, D. F. Eberl, and M. J. Welsh. 2009. TRPA channels distinguish gravity sensing from hearing in Johnston's organ. P Natl Acad Sci USA 106:13606-13611.

97. Riabinina, O., M. J. Dai, T. Duke, and J. T. Albert. 2011. Active Process Mediates SpeciesSpecific Tuning of Drosophila Ears. Curr Biol 21:658-664.

98. Manning, A. 1967. Antennae and Sexual Receptivity in Drosophila Melanogaster Females. Science 158:136-\&.

99. Matsuo, E., and A. Kamikouchi. 2013. Neuronal encoding of sound, gravity, and wind in the fruit fly. Journal of Comparative Physiology a-Neuroethology Sensory Neural and Behavioral Physiology 199:253-262.

100. Kavlie, R. G., M. J. Kernan, and D. F. Eberl. 2010. Hearing in Drosophila requires TilB, a conserved protein associated with ciliary motility. Genetics 185:177-188.

101. Gong, Z. F., W. S. Son, Y. D. Chung, J. W. Kim, D. W. Shin, C. A. McClung, Y. Lee, H. W. Lee, D. J. Chang, B. K. Kaang, H. W. Cho, U. Oh, J. Hirsh, M. J. Kernan, and C. S. Kim. 
2004. Two interdependent TRPV channel subunits, inactive and Nanchung, mediate hearing in Drosophila. J Neurosci 24:9059-9066.

102. Liang, X., J. Madrid, H. S. Saleh, and J. Howard. 2011. NOMPC, a Member of the TRP Channel Family, Localizes to the Tubular Body and Distal Cilium of Drosophila Campaniform and Chordotonal Receptor Cells. Cytoskeleton 68:1-7.

103. Gold, T. 1948. Hearing .2. The Physical Basis of the Action of the Cochlea. Proceedings of the Royal Society Series B-Biological Sciences 135:492-498.

104. Göpfert, M. C., J. T. Albert, B. Nadrowski, and A. Kamikouchi. 2006. Specification of auditory sensitivity by Drosophila TRP channels. Nature Neuroscience 9:999-1000.

105. Effertz, T., R. Wiek, and M. C. Göpfert. 2011. NompC TRP Channel Is Essential for Drosophila Sound Receptor Function. Curr Biol 21:592-597.

106. Guan, C. 2017. In Unpublished observation. University of Göttingen, Germany.

107. Quillin, K. J. 1999. Kinematic scaling of locomotion by hydrostatic animals: Ontogeny of peristaltic crawling by the earthworm Lumbricus terrestris. J Exp Biol 202:661-674.

108. Cacciatore, T. W., R. Rozenshteyn, and W. B. Kristan. 2000. Kinematics and modeling of leech crawling: Evidence for an oscillatory behavior produced by propagating waves of excitation. J Neurosci 20:1643-1655.

109. Suster, M. L., and M. Bate. 2002. Embryonic assembly of a central pattern generator without sensory input. Nature 416:174-178.

110. van Griethuijsen, L. I., and B. A. Trimmer. 2014. Locomotion in caterpillars. Biol Rev 89:656670.

111. Kuo, A. D. 2002. The relative roles of feedforward and feedback in the control of rhythmic movements. Motor Control 6:129-145.

112. Paoletti, P., and L. Mahadevan. 2014. A proprioceptive neuromechanical theory of crawling. P Roy Soc B-Biol Sci 281.

113. Simon, M. A., W. A. Woods, Y. V. Serebrenik, S. M. Simon, L. I. van Griethuijsen, J. J. Socha, W. K. Lee, and B. A. Trimmer. 2010. Visceral-Locomotory Pistoning in Crawling Caterpillars. Curr Biol 20:1458-1463.

114. Kernan, M., D. Cowan, and C. Zuker. 1994. Genetic Dissection of Mechanosensory Transduction - Mechanoreception-Defective Mutations of Drosophila. Neuron 12:1195-1206.

115. Hughes, C. L., and J. B. Thomas. 2007. A sensory feedback circuit coordinates muscle activity in Drosophila. Mol Cell Neurosci 35:383-396.

116. Heissler, S. M., K. Chinthalapudi, and J. R. Sellers. 2015. Kinetic characterization of the sole nonmuscle myosin-2 from the model organism Drosophila melanogaster. Faseb J 29:14561466.

117. Young, P. E., A. M. Richman, A. S. Ketchum, and D. P. Kiehart. 1993. Morphogenesis in Drosophila Requires Nonmuscle Myosin Heavy-Chain Function. Gene Dev 7:29-41.

118. Sellers, J. R. 2000. Myosins: a diverse superfamily. Bba-Mol Cell Res 1496:3-22.

119. Abreu-Blanco, M. T., J. M. Verboon, and S. M. Parkhurst. 2011. Cell wound repair in Drosophila occurs through three distinct phases of membrane and cytoskeletal remodeling. J Cell Biol 193:455-464.

120. Seabrooke, S., X. P. Qiu, and B. A. Stewart. 2010. Nonmuscle Myosin II helps regulate synaptic vesicle mobility at the Drosophila neuromuscular junction. Bmc Neurosci 11.

121. Monier, B., A. Pelissier-Monier, A. H. Brand, and B. Sanson. 2010. An actomyosin-based barrier inhibits cell mixing at compartmental boundaries in Drosophila embryos. Nat Cell Biol 12:60-U147.

122. Rauzi, M., P. F. Lenne, and T. Lecuit. 2010. Planar polarized actomyosin contractile flows control epithelial junction remodelling. Nature 468:1110-U1515.

123. Edwards, K. A., and D. P. Kiehart. 1996. Drosophila nonmuscle myosin II has multiple essential roles in imaginal disc and egg chamber morphogenesis. Development 122:14991511.

124. Wolfrum, U. 1990. Actin-Filaments - the Main Components of the Scolopale in Insect Sensilla. Cell and Tissue Research 261:85-96.

125. Wolfrum, U. 1992. Cytoskeletal Elements in Ciliary Receptor Systems. Cell Motility and the Cytoskeleton 23:311-311.

126. Wolfrum, U. 1997. Cytoskeletal elements in insect sensilla. International Journal of Insect Morphology \& Embryology 26:191-203. 
127. Ashkin, A. 1992. Forces of a Single-Beam Gradient Laser Trap on a Dielectric Sphere in the Ray Optics Regime. Biophys J 61:569-582.

128. Ashkin, A. 1978. Trapping of Atoms by Resonance Radiation Pressure. Phys Rev Lett 40:729-732.

129. Ashkin, A. 1998. Forces of a single-beam gradient laser trap on a dielectric sphere in the ray optics regime. Method Cell Biol 55:1-27.

130. Ashkin, A., J. M. Dziedzic, J. E. Bjorkholm, and S. Chu. 1986. Observation of a Single-Beam Gradient Force Optical Trap for Dielectric Particles. Opt Lett 11:288-290.

131. Schlosser, F. 2015. Mechanics of suspended cells probed by dual optical traps in a confocal microscope (PhD thesis). In Third Institute of Physics - Biophysics, Faculty of Physics. University of Göttingen, Germany.

132. Ashkin, A., J. M. Dziedzic, and T. Yamane. 1987. Optical Trapping and Manipulation of Single Cells Using Infrared-Laser Beams. Nature 330:769-771.

133. Wessel, A. D. 2015. Intracellular micromechanics of the syncytial Drosophila embryo (PhD thesis) In Third Institute of Physics - Biophysics, Faculty of Physics. University of Göttingen, Germany.

134. Block, S. M., D. F. Blair, and H. C. Berg. 1989. Compliance of Bacterial Flagella Measured with Optical Tweezers. Nature 338:514-518.

135. Konig, K., L. Svaasand, Y. G. Liu, G. Sonek, P. Patrizio, Y. Tadir, M. W. Berns, and B. J. Tromberg. 1996. Determination of motility forces of human spermatozoa using an $800 \mathrm{~nm}$ optical trap. Cell Mol Biol 42:501-509.

136. Svoboda, K., C. F. Schmidt, B. J. Schnapp, and S. M. Block. 1993. Direct Observation of Kinesin Stepping by Optical Trapping Interferometry. Nature 365:721-727.

137. Gittes, F., and C. F. Schmidt. 1998. Signals and noise in micromechanical measurements. Method Cell Biol 55:129-156.

138. Svoboda, K., and S. M. Block. 1994. Biological Applications of Optical Forces. Annu Rev Bioph Biom 23:247-285.

139. Edelstein, A. D., Tsuchida, M. A., Amodaj, N., Pinkard, H., Vale, R. D., Stuurman, N. 2014. Advanced methods of microscope control using $\mu$ Manager software. Journal of Biological Methods 1:e11.

140. Schneider, C. A., W. S. Rasband, and K. W. Eliceiri. 2012. NIH Image to ImageJ: 25 years of image analysis. Nat Methods 9:671-675.

141. Miller, J. C. e. 1994. Laser ablation: Principles and Applications. Springer Verlag, Berlin Heidelberg.

142. Vogel, A., and V. Venugopalan. 2003. Mechanisms of pulsed laser ablation of biological tissues (vol 103, pg 577, 2003). Chem Rev 103:2079-2079.

143. Maiman, T. H. 1960. Stimulated Optical Radiation in Ruby. Nature 187:493-494.

144. Campbell, C. J., M. C. Rittler, K. S. Noyori, C. H. Swope, and C. J. Koester. 1966. Threshold of Retina to Damage by Laser Energy. Arch Ophthalmol-Chic 76:437-\&.

145. Goldman, L., D. J. Blaney, D. J. Kindel, and E. K. Franke. 1963. Effect of the Laser Beam on the Skin. J Invest Dermatol 40:121-122.

146. Zweng, H. C., M. Flocks, N. A. Peppers, N. S. Kapany, and N. Silbertrust. 1964. Experimental Laser Photocoagulation. Am J Ophthalmol 58:353-+.

147. L'Esperance, F. A. J. 1968. An ophthalmic argon laser photocoagulation system: Design, construction and laboratory investigations. Transactions of the American Ophthalmological Society 66:827-904.

148. Krasnov, M. M. 1973. Laseropuncture of Anterior Chamber Angle in Glaucoma. Am J Ophthalmol 75:674-678.

149. Kiehart, D. P., C. G. Galbraith, K. A. Edwards, W. L. Rickoll, and R. A. Montague. 2000. Multiple forces contribute to cell sheet morphogenesis for dorsal closure in Drosophila. J Cell Biol 149:471-490.

150. WilliamsMasson, E. M., A. N. Malik, and J. Hardin. 1997. An actin-mediated two-step mechanism is required for ventral enclosure of the C-elegans hypodermis. Development 124:2889-2901.

151. Rauzi, M., P. Verant, T. Lecuit, and P. F. Lenne. 2008. Nature and anisotropy of cortical forces orienting Drosophila tissue morphogenesis. Nat Cell Biol 10:1401-U1457. 
152. Blanchet, G. B., J. B. Russell, C. R. Fincher, and M. Portmann. 1992. Laser Micromanipulation in the Mouse Embryo - a Novel-Approach to Zona Drilling. Fertil Steril 57:1337-1341.

153. Wood, W., A. Jacinto, R. Grose, S. Woolner, J. Gale, C. Wilson, and P. Martin. 2002. Wound healing recapitulates morphogenesis in Drosophila embryos. Nat Cell Biol 4:907-912.

154. Kumar, S., I. Z. Maxwell, A. Heisterkamp, T. R. Polte, T. P. Lele, M. Salanga, E. Mazur, and D. E. Ingber. 2006. Viscoelastic retraction of single living stress fibers and its impact on cell shape, cytoskeletal organization, and extracellular matrix mechanics. Biophys J 90:37623773.

155. Tanner, K., A. Boudreau, M. J. Bissell, and S. Kumar. 2010. Dissecting Regional Variations in Stress Fiber Mechanics in Living Cells with Laser Nanosurgery. Biophys J 99:2775-2783.

156. Stachowiak, M. R., and B. O'Shaughnessy. 2009. Recoil after Severing Reveals Stress Fiber Contraction Mechanisms. Biophys J 97:462-471.

157. Wu, J., R. B. Dickinson, and T. P. Lele. 2012. Investigation of in vivo microtubule and stress fiber mechanics with laser ablation. Integr Biol-Uk 4:471-479.

158. Wu, J., G. Misra, R. J. Russell, A. J. C. Ladd, T. P. Lele, and R. B. Dickinson. 2011. Effects of dynein on microtubule mechanics and centrosome positioning. Mol Biol Cell 22:48344841.

159. Maiato, H., C. L. Rieder, and A. Khodjakov. 2004. Kinetochore-driven formation of kinetochore fibers contributes to spindle assembly during animal mitosis. J Cell Biol 167:831840.

160. Colombelli, J., E. G. Reynaud, J. Rietdorf, R. Pepperkok, and E. H. K. Stelzer. 2005. In vivo selective cytoskeleton dynamics quantification in interphase cells induced by pulsed ultraviolet laser nanosurgery. Traffic 6:1093-1102.

161. Tinevez, J. Y., U. Schulze, G. Salbreux, J. Roensch, J. F. Joanny, and E. Paluch. 2009. Role of cortical tension in bleb growth. P Natl Acad Sci USA 106:18581-18586.

162. Reffay, M., L. Petitjean, S. Coscoy, E. Grasland-Mongrain, F. Amblard, A. Buguin, and P. Silberzan. 2011. Orientation and Polarity in Collectively Migrating Cell Structures: Statics and Dynamics. Biophys J 100:2566-2575.

163. Stramer, B., W. Wood, M. J. Galko, M. J. Redd, A. Jacinto, S. M. Parkhurst, and P. Martin. 2005. Live imaging of wound inflammation in Drosophila embryos reveals key roles for small GTPases during in vivo cell migration. J Cell Biol 168:567-573.

164. Haefner, S., O. Bamchen, and K. Jacobs. 2015. Capillary droplet propulsion on a fibre. Soft Matter 11:6921-6926.

165. Chen, T. W., T. J. Wardill, Y. Sun, S. R. Pulver, S. L. Renninger, A. Baohan, E. R. Schreiter, R. A. Kerr, M. B. Orger, V. Jayaraman, L. L. Looger, K. Svoboda, and D. S. Kim. 2013. Ultrasensitive fluorescent proteins for imaging neuronal activity. Nature 499:295-+.

166. Repiso, A., P. Saavedra, J. Casal, and P. A. Lawrence. 2010. Planar cell polarity: the orientation of larval denticles in Drosophila appears to depend on gradients of Dachsous and Fat. Development 137:3411-3415.

167. Li, Y. H., E. E. Treffers, S. Napthine, A. Tas, L. C. Zhu, Z. Sun, S. Bell, B. L. Mark, P. A. van Veelen, M. J. van Hemert, A. E. Firth, I. Brierley, E. J. Snijder, and Y. Fang. 2014. Transactivation of programmed ribosomal frameshifting by a viral protein. P Natl Acad Sci USA 111:E2172-E2181.

168. Akaike, H. 1974. New Look at Statistical-Model Identification. leee T Automat Contr Ac19:716-723.

169. Storm, C., J. J. Pastore, F. C. MacKintosh, T. C. Lubensky, and P. A. Janmey. 2005. Nonlinear elasticity in biological gels. Nature 435:191-194.

170. Carrillo, J. M. Y., F. C. MacKintosh, and A. V. Dobrynin. 2013. Nonlinear Elasticity: From Single Chain to Networks and Gels. Macromolecules 46:3679-3692.

171. Janmey, P. A., S. Hvidt, J. Kas, D. Lerche, A. Maggs, E. Sackmann, M. Schliwa, and T. P. Stossel. 1994. The Mechanical-Properties of Actin Gels - Elastic-Modulus and Filament Motions. J Biol Chem 269:32503-32513.

172. Orgogozo, V., F. Schweisguth, and Y. Bellaiche. 2001. Lineage, cell polarity and inscuteable function in the peripheral nervous system of the Drosophila embryo. Development 128:631643. 
173. Brangwynne, C. P., F. C. MacKintosh, S. Kumar, N. A. Geisse, J. Talbot, L. Mahadevan, K. K. Parker, D. E. Ingber, and D. A. Weitz. 2006. Microtubules can bear enhanced compressive loads in living cells because of lateral reinforcement. J Cell Biol 173:733-741.

174. Kovacs, M., J. Toth, C. Hetenyi, A. Malnasi-Csizmadia, and J. R. Sellers. 2004. Mechanism of blebbistatin inhibition of myosin II. J Biol Chem 279:35557-35563.

175. Limouze, J., A. F. Straight, T. Mitchison, and J. E. Sellers. 2004. Specificity of blebbistatin, an inhibitor of myosin II. J Muscle Res Cell M 25:337-341.

176. Straight, A. F., A. Cheung, J. Limouze, I. Chen, N. J. Westwood, J. R. Sellers, and T. J. Mitchison. 2003. Dissecting temporal and spatial control of cytokinesis with a myosin II inhibitor. Science 299:1743-1747.

177. Ishizaki, T., M. Uehata, I. Tamechika, J. Keel, K. Nonomura, M. Maekawa, and S. Narumiya. 2000. Pharmacological properties of Y-27632, a specific inhibitor of Rho-associated kinases. Mol Pharmacol 57:976-983.

178. Sommi, P., D. Cheerambathur, I. Brust-Mascher, and A. Mogilner. 2011. ActomyosinDependent Cortical Dynamics Contributes to the Prophase Force-Balance in the Early Drosophila Embryo. Plos One 6.

179. Simoes, S. D., J. T. Blankenship, O. Weitz, D. L. Farrell, M. Tamada, R. FernandezGonzalez, and J. A. Zallen. 2010. Rho-Kinase Directs Bazooka/Par-3 Planar Polarity during Drosophila Axis Elongation. Dev Cell 19:377-388.

180. Escudero, L. M., M. Bischoff, and M. Freeman. 2007. Myosin II regulates complex cellular arrangement and epithelial architecture in drosophila. Dev Cell 13:717-729.

181. Krajcovic, M. M., and J. S. Minden. 2012. Assessing the critical period for Rho kinase activity during Drosophila ventral furrow formation. Dev Dynam 241:1729-1743. 


\section{CURRICULUM VIT/E: Achintya Prahlad}

\section{Personal Data}

Place and Date of Birth:

Nationality:

Address:

Email:

Phone:
Bengaluru, India, 17 May 1991

Indian

Theodor-Heuss-Straße 26, Apt 25

37075 Göttingen, Germany

achintya.prahlad@gmail.com

+4917671952810

\section{Education}

2013-present: Doctoral Candidate

Third Institute of Physics - Biophysics (DPI)

University of Göttingen

Specialization:

Biophysics

Dissertation:

"Mechanical properties of the Ich5 organ in the Drosophila larva"

Advisor:

Prof. Dr. Christoph F. Schmidt

2011-2013:

MSc in Neuroscience

University of Göttingen

Thesis:

Advisor:

"Behaviour of the Protein TDP-43 in solution"

Prof. Dr. Mikael Simons, MPI-em

2008-2011: $\quad$ BSc Honours in Chemistry

Delhi University, Delhi

\section{Publication (under consideration)}

"Mechanical properties of a Drosophila larval chordotonal organ", Prahlad, A., C. Spalthoff, D. Kong, J. Großhans, M. C. Göpfert, and C. F. Schmidt. 2017, Biophysical Journal, under revision.

\section{Scholarships and certificates}

- IMPRS stipend recipient during Masters studies

- St. Stephen's College-Sumitomo Scholarship.

- K. Swaminathan Memorial Prize, St Stephens College, 2009-10, 2010-11.

- Science Meritorious Prize from Delhi University (all 3 years of undergraduate studies).

- INSPIRE scholarship of the Department of Science and Technology, Government of India, for outstanding performance in the All India Engineering Entrance Examination (AIEEE) 2008. 
- Graduate Record Examination (GRE): Verbal 163 (91st percentile): Quantitative reasoning: 164 (90th percentile).

\section{Posters presented in}

- Annual meeting, American Society for Cell Biology (ASCB), Dec 2016 San Francisco, USA.

- Annual meeting, Deutsche Physikalische Gesellschaft (German Physical Society) 2016, 2015, 2014.

- Physics of the Cell: From Molecules to Systems (PhysCell 2015, EMBO Conference and Advanced School) 30 Aug - 4 Sep 2015, Bad Staffelstein, Germany.

\section{Other interests}

Music and languages

\section{Languages}

Tamil, Kannada, Hindi/Urdu, Bangla, Marathi, English. Working knowledge of German. 